\title{
GUIDANCE OF RECEIVER AIRCRAFT TO RENDEZVOUS WITH TANKER IN THE PRESENCE OF WIND
}

\author{
by \\ JANE-WIT KAMPOON
}

Presented to the Faculty of the Graduate School of

The University of Texas at Arlington in Partial Fulfillment

of the Requirements

for the Degree of

MASTER OF SCIENCE IN AEROSPACE ENGINEERING

THE UNIVERSITY OF TEXAS AT ARLINGTON

December 2009 


\section{ACKNOWLEDGEMENTS}

I would like to thank my supervising professor Dr. Atilla Dogan for constantly motivating and encouraging me, and also for his invaluable advice during the course of my studies. I wish to thank my academic advisors Dr. Donald R. Wilson and Dr. Kamesh Subbarao for their interest in my research and for taking time to serve in my thesis committee.

I would also like to extend my appreciation to Royal Thai Air Force for providing scholarship for my Master studies. I wish to thank all my colleagues and co-workers at Aeronautical and Aviation Engineering Department, Royal Thai Air Force Academy for their support and encouragement.

Finally, I would like to express my extreme gratitude to my parents, sister and wife for their sacrifice, encouragement and patience. I also thank several of my friends, especially Dr.Natee Pantong who have helped me throughout my career.

December 7, 2009 


\begin{abstract}
GUIDANCE OF RECEIVER AIRCRAFT TO RENDEZVOUS WITH TANKER IN THE PRESENCE OF WIND

JANE-WIT KAMPOON, M.S.

The University of Texas at Arlington, 2009
\end{abstract}

Supervising Professor: Atilla Dogan

This thesis work addresses the problem of aircraft rendezvous in the presence of prevailing wind for automated aerial refueling operations. A modified point-parallel rendezvous procedure is defined. The tanker aircraft performs a racetrack maneuver along a pre-specified refueling orbit. The receiver aircraft enters the refueling area through a fixed point and flies along the refueling line that is aligned with one of the straight legs of the race track. It is the responsibility of the receiver to meet the tanker at the rendezvous point. A virtual target is used to define the trajectory of the refueling orbit. A nonlinear guidance algorithm is used to generate yaw rate commands for the tanker to follow the virtual target. An existing low-level controller is employed for the tanker to track the commanded yaw rate as well as commanded airspeed and altitude. The receiver aircraft has a controller to track a commanded trajectory relative to a moving reference frame. The concept of virtual tanker is introduced to synchronize the motion of the receiver along the refueling line with the tanker flying on the refueling orbit. The distance of the virtual tanker along the straight refueling line to the rendezvous point is synchronized with the distance-to- 
travel by the tanker to arrive the same point. The trajectory tracking controller of receiver flies the receiver at the precontract position relative to the virtual tanker until the virtual tanker rendezvous with the actual tanker. Then, the receiver switches to following the precontract position relative to the actual tanker. This establishes the aerial refueling formation of the aircraft. The guidance algorithms are implemented in a high fidelity simulation environment that includes the 6-DOF nonlinear models of both aircraft with terms for the dynamics effect of wind as well as the low-level controllers. The simulation of the refueling rendezvous in a realistic prevailing wind demonstrates that the nonlinear guidance logic coupled with the low-level controller is capable of flying the tanker along the refueling orbit. Further, the guidance algorithm based on the virtual tanker concept along with the trajectory tracking controller can successfully achieve the rendezvous of the receiver with the tanker even in the presence of strong time-varying prevailing wind. 


\section{TABLE OF CONTENTS}

ACKNOWLEDGEMENTS ................... ii

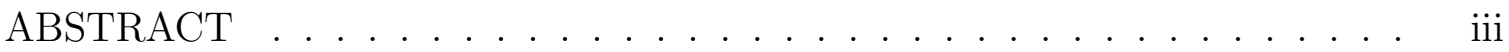

LIST OF FIGURES $\ldots \ldots \ldots \ldots \ldots \ldots \ldots$ viii

Chapter $\quad$ Page

1. INTRODUCTION ........................ 1

1.1 Background and motivation . . . . . . . . . . . . . 1

1.2 Related research . . . . . . . . . . . . . . . . . . . . 4

1.3 Thesis overview . . . . . . . . . . . . . . . . . . . . 7

2. PROBLEM DESCRIPTION . . . . . . . . . . . . . . . . 8

2.1 Introduction . . . . . . . . . . . . . . . . . . . 8

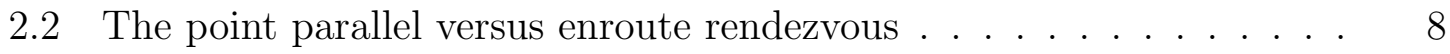

2.3 Rendezvous requirements . . . . . . . . . . . . . . . . . . . . . 10

2.4 Tanker aircraft model and controller . . . . . . . . . . . . . . 11

2.4.1 Translational kinematics equations . . . . . . . . . . . . 11

2.4.2 Translational dynamics equations . . . . . . . . . . . . 11

2.4.3 Rotational kinematics equations . . . . . . . . . . . . . . . 12

2.4.4 Rotational dynamics equations . . . . . . . . . . . . . 12

2.4.5 Engine dynamics . . . . . . . . . . . . . . . . . . . 13

2.4 .6 Actuator dynamics . . . . . . . . . . . . . . 13

2.4 .7 Tanker controller . . . . . . . . . . . . . . . . . . . . . 13

2.5 Receiver aircraft model and controller . . . . . . . . . . . . . . 15

2.5.1 Translational kinematics equations _. . . . . . . . . 15 
2.5.2 Translational dynamics equations . . . . . . . . . . . 16

2.5.3 Rotational kinematics equations . . . . . . . . . . . 16

2.5.4 Rotation dynamics equations . . . . . . . . . . . . 17

2.5.5 Engine dynamics . . . . . . . . . . . . . . . . . . . 17

2.5.6 Actuator dynamics . . . . . . . . . . . . . . . . . . 18

2.5.7 Receiver controller . . . . . . . . . . . . . . . . . 18

3. GUIDANCE ALGORITHMS . . . . . . . . . . . . . . . . . 20

3.1 Introduction . . . . . . . . . . . . . . . . 20

3.2 Tanker aircraft . . . . . . . . . . . . . . . 20

3.2.1 Virtual target trajectory . . . . . . . . . . . . . 21

3.2.2 Nonlinear guidance logic for virtual target tracking . . . . . . 23

3.2.3 Proportional speed control for tanker aircraft . . . . . . . . . 26

3.3 Receiver aircraft . . . . . . . . . . . . . . . . 27

4. SIMULATION AND ANALYSIS OF THE RESULTS . . . . . . . . . . . 36

4.1 Introduction . . . . . . . . . . . . . . . . 36

4.2 Tanker aircraft . . . . . . . . . . . . . . . 36

4.2.1 No wind condition . . . . . . . . . . . . . . . 37

4.2.2 Wind condition . . . . . . . . . . . . . . . . . . 49

4.3 Rendezvous of receiver aircraft with tanker . . . . . . . . . . 56

4.3.1 Description of rendezvous simulation . . . . . . . . . 56

4.3.2 No wind condition . . . . . . . . . . . . . . 61

4.3.3 Wind condition . . . . . . . . . . . . . . 72

5. CONCLUSION AND RECOMMENDATION FOR FUTURE WORK . . . 84 Appendix

A. THE AERIAL REFUELING TERMINOLOGY . . . . . . . . . . . . . 88

B. SCALAR EQUATIONS OF MOTION OF THE TANKER . . . . . . . . . 90 
C. SCALAR EQUATIONS OF MOTION OF THE RECEIVER . . . . . . . . 95

REFERENCES . . . . . . . . . . . . . . . . . . 103

BIOGRAPHICAL STATEMENT . . . . . . . . . . . . . . . . 106 


\section{LIST OF FIGURES}

Figure $\quad$ Page

1.1 Air refueling anchor ..................... . . . 2

1.2 Fighter turn-on air refueling . . . . . . . . . . . . . . . . 3

1.3 Point parallel rendezvous . . . . . . . . . . . . . . . . . . . . . 3

1.4 Enroute rendezvous . . . . . . . . . . . . . . . . . . . 4

2.1 Air refueling anchor . . . . . . . . . . . . . . . 9

2.2 The tanker's control diagram . . . . . . . . . . . . . . . . . 14

2.3 The receiver's control diagram . . . . . . . . . . . . . . . . 18

3.1 Diagram of virtual target trajectory . . . . . . . . . . . . . . 22

3.2 Diagram of nonlinear guidance logic . . . . . . . . . . . . . . . . . . 24

3.3 The relationship of lateral acceleration and commanded yaw rate . . . 26

3.4 Diagram of the virtual tanker aircraft trajectory . . . . . . . . . . 28

3.5 Diagram in the rendezvous turn . . . . . . . . . . . . . . . 29

3.6 Diagram in the rendezvous turn . . . . . . . . . . . . . . 30

3.7 Diagram in the rendezvous . . . . . . . . . . . . . . 31

3.8 Diagram of rendezvous trajectory . . . . . . . . . . . . . . 34

4.1 Tanker trajectory comparison with wind and no wind . . . . . . . . 37

4.2 Trajectory comparison when tanker initially start at (a) $(-5000,0)(\mathrm{b})$ $(-5000,100)(\mathrm{c})(-5000,500)$ and $(\mathrm{d})(-5000,1000) \ldots . . . . . .40$

4.3 Control deflection when tanker initially start at (a) $(-5000,0)$ (b) $(-5000,100)(\mathrm{c})(-5000,500)$ and $(\mathrm{d})(-5000,1000) \ldots . . . .$.

4.4 Comparison of yaw rate when tanker initially start at (a) $(-5000,0)$ (b) $(-5000,100)($ c) $(-5000,500)$ and $($ d $)(-5000,1000) \ldots . . .$. 
4.5 Comparison of commanded velocity when tanker initially start at (a) $(-5000,0)(\mathrm{b})(-5000,100)(\mathrm{c})(-5000,500)$ and $(\mathrm{d})(-5000,1000) \ldots$

4.6 Trajectory comparison when (a) $L 1=100$ (b) $L 1=500$ (c) $L 1=1000$ and $($ d) $L 1=2000 \ldots \ldots \ldots \ldots \ldots \ldots \ldots$

4.7 Control deflection when (a) $L 1=100$ (b) $L 1=500$ (c) $L 1=1000$ and (d) $L 1=2000 \ldots \ldots \ldots \ldots \ldots \ldots$

4.8 Comparison of yaw rate when (a) $L 1=100$ (b) $L 1=500$ (c) $L 1=1000$ and $($ d) $L 1=2000 \ldots \ldots \ldots \ldots \ldots \ldots \ldots$

4.9 Comparison of commanded velocity when (a) $L 1=100$ (b) $L 1=500$ (c) $L 1=1000$ and (d) $L 1=2000 \ldots \ldots \ldots \ldots \ldots$

4.10 Wind component versus time . . . . . . . . . . . . . 50

4.11 Trajectory comparison when (a) $K p=1$ (b) $K p=0.1$ (c) $K p=0.01$

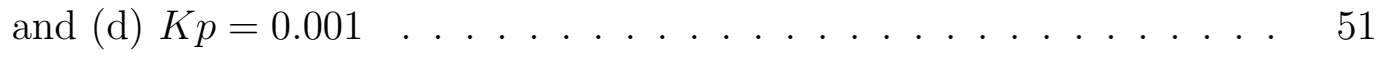

4.12 Trajectory comparison between tanker and virtual target . . . . . . 52

4.13 Comparison of position between tanker and virtual target . . . . . 52

4.14 Relative position between tanker and virtual target $\ldots \ldots \ldots . . .53$

4.15 Control signals command . . . . . . . . . . . . . . . . 53

4.16 Commanded velocity versus actual velocity . . . . . . . . . . 54

4.17 Commanded yaw rate versus actual yaw rate . . . . . . . . . 54

4.18 Yaw angle time history $\ldots \ldots \ldots \ldots \ldots$

4.19 Rendezvous initial position of the aircrafts $\ldots \ldots \ldots \ldots$

4.20 Block diagram for rendezvous simulation $\ldots \ldots \ldots \ldots$

4.21 Trajectory comparison of the aircrafts . . . . . . . . . . . 61

4.22 Tanker versus virtual target trajectory in $\mathrm{x}-\mathrm{y}$ axis $\ldots \ldots 62$

4.23 Tanker versus virtual target relative position along $\mathrm{x}-\mathrm{y}$ axis $\ldots \ldots 63$

4.24 Tanker versus virtual tanker position trajectory in $\mathrm{x}-\mathrm{y}$ axis $\ldots . . .64$

4.25 Tanker aircraft inertial velocities . . . . . . . . . . . . . . 65

4.26 Receiver aircraft inertial velocities . . . . . . . . . . . . 66 
4.27 Tanker aircraft Euler's angle . . . . . . . . . . . . . . . . 67

4.28 Receiver aircraft Euler's angle . . . . . . . . . . . . . . . 68

4.29 Tanker aircraft control signals . . . . . . . . . . . . . . . . 69

4.30 Receiver aircraft control signals . . . . . . . . . . . . . . . . . 70

4.31 Receiver aircraft's relative position . . . . . . . . . . . . . . 71

4.32 Trajectory comparison of the aircrafts . . . . . . . . . . . . 73

4.33 Tanker versus virtual target trajectory in $\mathrm{x}-\mathrm{y}$ axis . . . . . . . . . . 74

4.34 Tanker versus virtual target position difference along $\mathrm{x}-\mathrm{y}$ axis . . . . . 75

4.35 Tanker versus virtual tanker position trajectory in $\mathrm{x}-\mathrm{y}$ axis . . . . . . 76

4.36 Tanker aircraft inertial velocities . . . . . . . . . . . . . . . 77

4.37 Receiver aircraft inertial velocities . . . . . . . . . . . . . 78

4.38 Tanker aircraft Euler's angle . . . . . . . . . . . . . . . . 79

4.39 Receiver aircraft Euler's angle . . . . . . . . . . . . . . . . . 80

4.40 Tanker aircraft control signals . . . . . . . . . . . . . . 81

4.41 Receiver aircraft control signals . . . . . . . . . . . . . . 82

4.42 Receiver aircraft's relative position . . . . . . . . . . . . . . . 83 


\section{CHAPTER 1}

\section{INTRODUCTION}

\subsection{Background and motivation}

The benefits of UAVs to complete the missions beyond human ability are already realized for many years not only in military but also in civilian applications. However, it is desirable that they stay continuously airborne performing their missions even with limited fuel capacity. Recently, there is increased interest in solving various kinds of multiple aircraft rendezvous problems. Many valuable capabilities including aerial refueling are enabled by the rendezvous technologies. Currently, aerial refueling is done manually and requires well-trained pilots [1]. Aerial refueling is an important task for mission success in modern aerial operations. There are two ways of refueling: flying boom method and probe-and-drogue method. Between the two, they both have some advantages and disadvantages as mentioned in [2]. In either case, it would be better if the receiver aircraft were automatically controlled for aerial refueling. Many countries have realized the capabilities of the UAVs specifically in military applications. One area of expansion has been an increased level of autonomy. For example, some UAV systems are capable of performing their preprogrammed missions without any intervention. An important aspect of autonomy for UAVs would be the ability to perform aerial refueling, however, at this time, UAVs are not capable of aerial refueling.

Aerial refueling will extend the range, shorten the response time, and extend loiter time of UAVs. Additionally, it will lessen the logistical effort necessary to deploy them by allowing fewer assets to perform the same mission and reducing the need for 
forward basing. Aerial refueling will greatly increase the capability of UAVs while allowing them to retain their small size and light weight.

However, the question of how the receiver and tanker aircraft will join up together or rendezvous before initiating aerial refueling is one of the interesting research topics. For piloted aircraft, prior to transfer of fuel, the aircraft must first rendezvous. There are two general ways aircraft involved in aerial refueling rendezvous, anchor or track [3]. The anchor aerial refueling is defined in Ref. [4] as, the air refueling that is performed as the tankers maintain a prescribed pattern which is anchored to a geographical point. This refueling method is used typically when the amount of airspace available is restricted. When employing anchor aerial refueling, the tanker flies a small racetrack, while the receiver flies inbound on one of the straight legs of the racetrack; the tanker then rolls out in front of the receiver, as depicted in Fig 1.1. After rendezvous, the tanker flies a larger racetrack. For highly maneuverable receivers, such as fighter aircraft, the tanker aircraft will fly a highly predictable pattern and the receiver aircraft will perform the rendezvous. This type of rendezvous is referred to as a fighter turn-on, as shown in Fig 1.2.

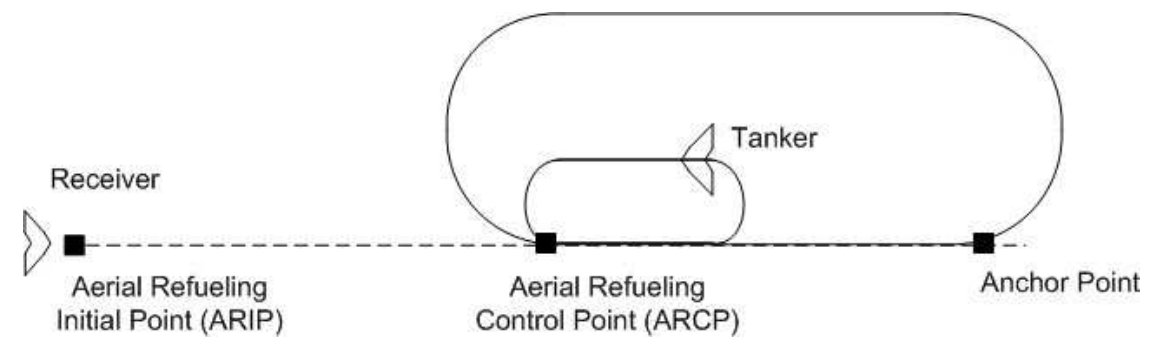

Figure 1.1. Air refueling anchor.

During large scale refueling operations, the tanker may continually fly the larger racetrack and receivers will perform rendezvous. Aerial refueling tracks are a series 


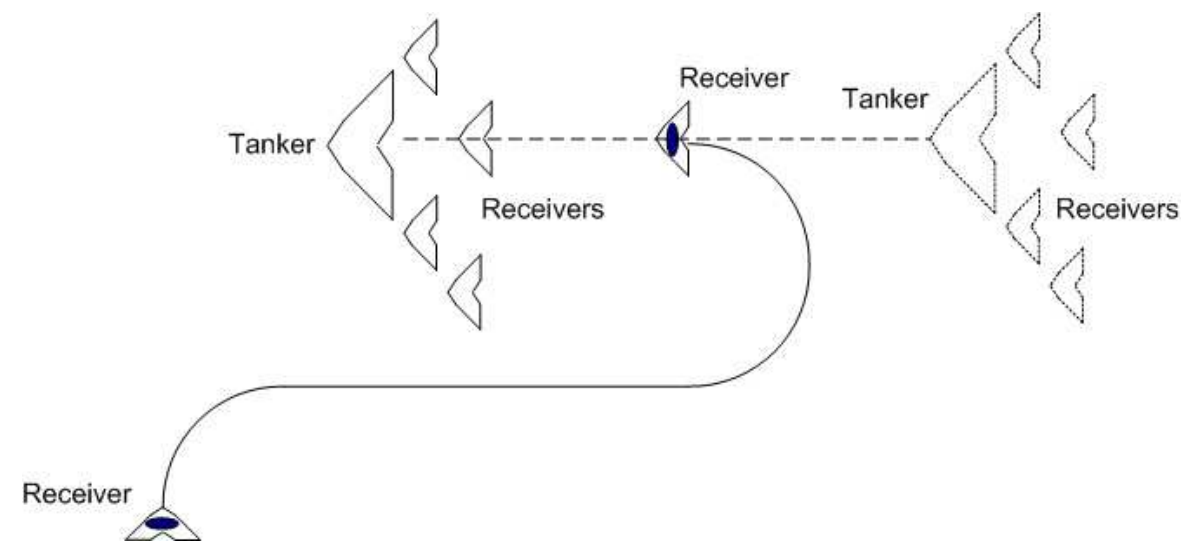

Figure 1.2. Fighter turn-on air refueling.

of waypoints, usually located along the receiver's planned route of flight. With track aerial refueling, rendezvous is accomplished in two different ways. The first method is point parallel, in which the tanker orbits about a designated point, called the arial refueling control point (ARCP), and waits for the receiver to arrive, then will roll out in front of the receiver. ARCP and various other terminologies of aerial refueling are listed in Appendix A. The second method is enroute, the tanker and receiver arrive simultaneously at the ARCP, as depicted in Fig 1.3. It should also be noted that the receiver does not directly intercept the tanker, but a point directly behind the tanker (approximately 1-3 nm). Once the receiver has achieved this position it must wait until cleared by the tanker to approach the pre-contact position [5]. However,

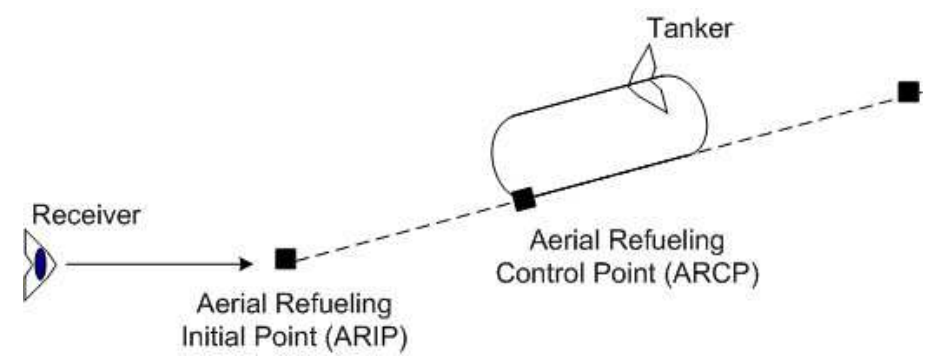

Figure 1.3. Point parallel rendezvous. 
the first step before performing aerial refueling, both the tanker and receiver aircraft must initiate the formation flight or rendezvous at designated points during the route. There are several factors for a rendezvous procedure to be successful, which might be different from one procedure to the other. Nonetheless, navigation and timing problems seem to be the common factors for every rendezvous procedure. The solution for the navigation is to answer the question of where shall we meet and the solution for the timing is to answer the question of when they shall meet. Moreover, in a realistic operation, the aircraft will encounter various unexpected factors from the atmosphere, such as wind gust, turbulence, etc. which make it much more difficult to perform a successful rendezvous. Thus, it is desirable to develop guidance algorithms based on the existing rendezvous procedure for the realistic operations of UAVs.

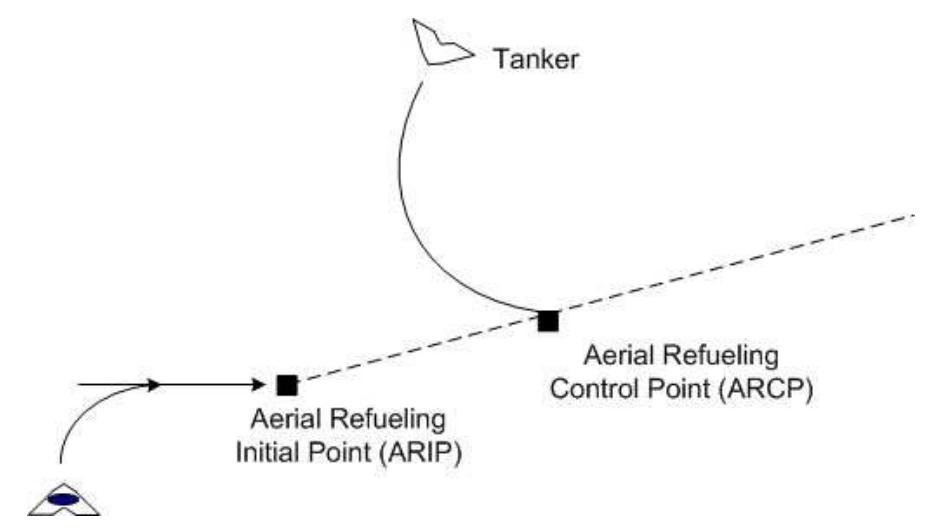

Figure 1.4. Enroute rendezvous.

\subsection{Related research}

Very little that is published involves the rendezvous problem of aircraft. In Ref. [1], a flight control system was developed utilizing the modification of proportional guidance logic for tightly tracking a desired flight path to rendezvous of two small 
UAVs. Ref. [2] proposed a flight control system design, based upon proportional navigation guidance and line of sight angle control, for receiver already in a position behind the tanker. Ref. [6] researched utilization of proportional navigation guidance with adaptive terminal guidance in order to enhance rendezvous performance by using a tanker estimator to predict the rendezvous location and proportional navigation to create a heading rate command to align the UAV's heading with the tanker prior to rendezvous. For the ability to autonomously rendezvous with the tanker, two problems were examined. The first problem is for the UAV receiver to rendezvous in minimum time with a known tanker path. The second problem is for the receiver to rendezvous at a specified time with a known tanker path. The determination of the control required to fly an optimum rendezvous using numerical optimization and to design a feedback controller that will approximate that optimal control to bring the receiver in the mile-in-tail position or a point $1 \mathrm{~nm}$ right behind the tanker was studied in Ref. [5]. Ref. [7] designed the trajectories from arbitrary initial position, for the Mini, a large scaled model airplane, to go to a $15 \mathrm{~m}$ location behind the Parent, which is moving in a circular flight path with constant speed $20 \mathrm{~m} / \mathrm{s}$. The Parent is a large scaled model airplane. This trajectory planner is implemented as the guidance logic in real hardware for flight testing. Ref. [8] studied one such problem of path planning and trajectory tracking for the surveillance of multiple locations in the presence of wind, the surveillance of multiple waypoints by an aircraft that flies with constant speed. It is assumed that the aircraft has a maximum turning rate and that the wind is equal to a known constant plus small possibly time varying components. The path planning is done by calculating the shortest time path through all of the waypoints in the presence of a known constant wind. During the calculation, the allowed turning rate is assumed to be less than the actual maximum turning rate of the vehicle. This algorithm produces a ground path that can be tracked by the control algorithm. The 
control algorithm breaks the desired trajectory into smaller sections, each of which can be approximated by a polynomial. A spacial sliding surface controller is then used to track each polynomial in the presence of unknown wind disturbances. Ref. [9] presents a new method for unmanned aerial vehicle path following using vector fields to represent desired ground track headings to direct the vehicle onto the desired path. The key feature of this approach is that ground track heading error and lateral following error approach zero asymptotically even in the presence of constant wind disturbances. Methods for following straight-line and circular orbit paths, as well as combinations of straight lines and arcs, are presented. Experimental results validate the effectiveness of this path following approach for small air vehicles flying in highwind conditions. Ref. [10] explored the problem of finding the optimal path from an initial position and orientation to a final position and orientation for an aircraft with a limited turning radius in the presence of a constant wind. An iterative method for solving for the paths has also been presented and several examples of optimal paths in the presence of wind have been presented.

An integrated simulation environment is developed to take into account tanker maneuvers, motion of the receiver relative to the tanker and the aerodynamic coupling due to the trailing wake-vortex of the tanker [11]. The simulation employs a full 6-DOF nonlinear mathematical model of the tanker aircraft. Additionally, the receiver dynamics is modeled utilizing the new set of equations derived to explicitly formulate the translational and rotational motion of the receiver relative to the tanker. Further, the equations have explicit terms that incorporate the wind effects in the translational and rotational dynamics and kinematics [12]. The separate dynamic model of the tanker, including its own controller for the racetrack maneuvers of the tanker is used. A LQR-based MIMO state-feedback and integral control is developed to track commanded speed, altitude and yaw rate. Similarly, for the relative 
motion of the receiver, an LQR-based MIMO state-feedback and integral control is designed to track commanded trajectory expressed in the body frame of the tanker [13]. Both controllers schedule their corresponding feedback and integral gains based on the commanded speed and yaw rate of the tanker [14].

The research work in this thesis applies prior works on mathematical modeling of relative motion [11], [12], [13], aerodynamics coupling [14] and the proportional navigation guidance logic [1] to the realistic simulation environment of aerial refueling rendezvous procedure in the presence of wind to demonstrate its feasibility.

\subsection{Thesis overview}

This paper is organized as follows. chapter 2 discusses the system model and provides the problem formulation. Additionally, the point parallel and enroute rendezvous procedures for aerial refueling is described. chapter 3 details guidance algorithms for both tanker and receiver aircraft. chapter 4 presents the results of the simulations. chapter 5 gives the conclusions and recommendations for future work. 


\section{CHAPTER 2}

\section{PROBLEM DESCRIPTION}

\subsection{Introduction}

This chapter gives the description of the problem that this thesis research work attempts to solve. The objectives of this research work are to develop a procedure for UAVs to rendezvous with the tanker aircraft for aerial refueling in the presence of strong prevailing wind and demonstrate the feasibility of the procedure in an integrated simulation environment. The next section describes two common aerial refueling rendezvous procedures and gives the explanation of the choice for this study. section 2.3 presents the requirements for the UAV rendezvous approach based on the selected rendezvous procedure along with the aircraft models and the controllers already available from prior work. The last two sections describe the models and the controllers used in the integrated simulation environment. The characteristics of the prevailing wind and the vortex induced wind are also presented in these sections.

\subsection{The point parallel versus enroute rendezvous}

The two most commonly used air refueling rendezvous procedures are called the point parallel and the enroute. The point parallel rendezvous is an air refueling rendezvous where the tanker aircraft orbits at the ARCP until the receiver crosses the Air Refueling Initial Point (ARIP) flying toward the ARCP. The Air Refueling Control Time (ARCT) is used for planning the rendezvous, The tanker aircraft enters an orbit pattern as shown Fig 2.1, described as a racetrack pattern to the left using 
2 minute legs (approximately $14 \mathrm{~nm}$ in length) Once the tanker is positioned in front of the receiver, the receiver begins a gentle closure to the pre-contact position.

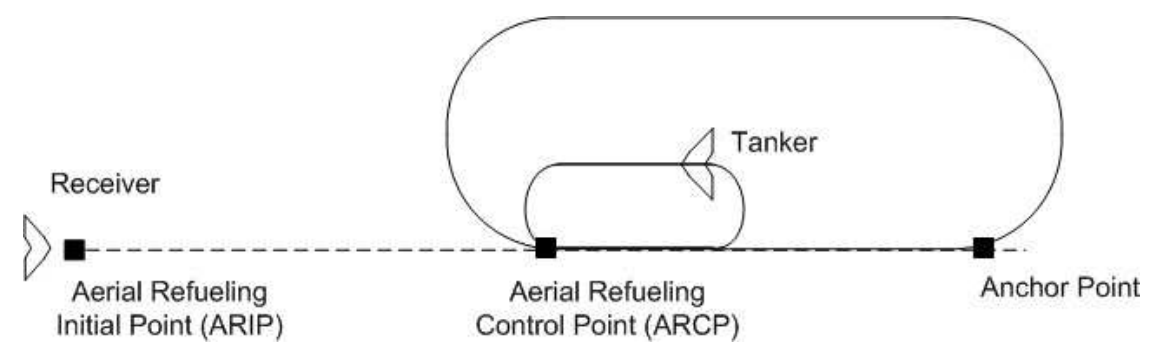

Figure 2.1. Air refueling anchor.

The enroute rendezvous differs from the point parallel rendezvous in that there is no specified tanker orbit in which the tanker awaits the receiver. The tanker and receiver aircraft fly to an ARIP within one minute of one another and then along a common track to the ARCP. [15].

Either of the two primary air refueling rendezvous, the point parallel or the enroute, are compatible with the UAV systems. However, the modified point parallel rendezvous is implemented in this work because of a few advantages. This procedure can be accomplished in a restricted airspace and can accommodate multiple receivers coming from various directions.

In this procedure, as described in [15], there are two minor modifications to the standard procedure to permit the rendezvous to be accomplished despite the potentially hostile environment. First, the replacement of a standard tanker orbit with a timed orbit which allows the tanker to plan an orbit pattern to arrive at the ARCP at exactly the ARCT. The second modification changes the distance between aircraft at the end of the rendezvous. Normally, the tanker will complete its rendezvous turn 1-3 $\mathrm{nm}$ in front of the receiver. 


\subsection{Rendezvous requirements}

Guidance algorithms should be developed for the receiver and the tanker for the implementation of the modified point parallel rendezvous procedure. In this rendezvous procedure, the receiver should meet the tanker aircraft at the ARCP right after the tanker completes its turn. When the two aircraft join and establish the formation, the receiver aircraft should be at the observation or pre-contact position relative to the tanker. This procedure requires the exact timing for both aircraft to arrive at the $\mathrm{ARCP}$ at the same time. In the modified point parallel rendezvous procedure, the tanker aircraft is required to fly at a constant altitude in a racetrack trajectory that is fixed in the inertial frame and attached to the ARCP as shown in Fig. 2.1. In the presence of prevailing wind that is spacially and temporally varying, the ground speed of the tanker may vary, which, in turn, changes the time of arrival at the rendezvous point. Further, at the time of arrival, the orientation, heading and flight path angle of the tanker may not be perfectly aligned with the straight line that joins the ARIP and ARCP. In the modified point parallel rendezvous procedure, it is the responsibility of the receiver aircraft to arrive at the $\mathrm{ARCP}$ on time and initiate the formation at the pre-contact position despite the effect of the prevailing wind on its and tanker's motion.

Another important requirement for both receiver and the tanker is that the guidance algorithm of each aircraft should be based on the current controllers already designed and implemented in the integrated simulation environment. The controller of the tanker tracks the commanded airspeed, altitude and yaw rate. Thus, the guidance algorithm of the tanker should generate commanded airspeed, altitude and yaw rate such that the aircraft flies on the refueling orbit as defined earlier. On the other hand, the controller of the receiver tracks commanded position relative to the tanker in the tanker's body-fixed frame. Thus, the guidance algorithm of the receiver 
should generate position commands such that the receiver arrives at the ARCP and initiates the formation at the pre-contact position with out excessive transients.

2.4 Tanker aircraft model and controller

The full dynamic model of the tanker aircraft derived in a previous work by [16] and the controller designed and validated by [17] are detailed in this section.

\subsubsection{Translational kinematics equations}

The translational kinematics equation is written in terms of the position vector of the tanker with respect to an inertial frame. In matrix form, the translational kinematics equation is

$$
\dot{r}_{B_{T}}=\mathbf{R}_{\mathbf{B}_{\mathbf{T}} \mathbf{T}}^{\mathbf{T}} \mathbf{R}_{\mathbf{B}_{\mathbf{T}} \mathbf{w}_{\mathbf{T}}} V_{w_{T}}
$$

where $r_{B_{T}}$ is the position of the tanker relative to the inertial frame expressed in the inertial frame, $\mathbf{R}_{\mathbf{B}_{\mathbf{T}} \mathbf{I}}$ is the rotation matrix from the inertial frame to the body frame of the tanker, $\mathbf{R}_{\mathbf{B}_{\mathbf{T}} \mathbf{w}_{\mathbf{T}}}$ is the rotation matrix from the tanker wind frame to body frame, $V_{w_{T}}$ is the velocity of the tanker relative to the surrounding air expressed in the tanker wind frame. The scalar form of translational kinematics equations used in this thesis are presented in appendix B.

\subsubsection{Translational dynamics equations}

Translational dynamics equation of the tanker aircraft in matrix form is 


$$
\left[\begin{array}{c}
\dot{V}_{T} \\
\dot{\beta}_{T} \\
\dot{\alpha}_{T}
\end{array}\right]=\mathcal{E}_{\mathbf{T}}^{-1} \mathbf{S}\left(\omega_{\mathbf{B}_{\mathbf{T}}}\right) \mathbf{R}_{\mathbf{B}_{\mathbf{T}} \mathbf{w}_{\mathbf{T}}} V_{w_{T}}+\frac{1}{m_{T}} \mathcal{E}_{\mathbf{T}}^{-\mathbf{1}}\left(\mathbf{R}_{\mathbf{B}_{\mathbf{R}} \mathbf{I}} M_{T} \mathbf{R}_{\mathbf{B}_{\mathbf{T}} \mathbf{w}_{\mathbf{T}}} A_{T}+P_{T}\right)
$$

where

$$
\mathcal{E}_{\mathbf{T}}^{-1}=\left[\begin{array}{ccc}
\cos \alpha_{T} \cos \beta_{T} & \sin \beta_{T} & \cos \beta_{T} \sin \alpha_{T} \\
-\frac{1}{V_{T}} \cos \alpha_{T} \sin \beta_{T} & \frac{1}{V_{T}} \cos \beta_{T} & -\frac{1}{V_{T}} \sin \alpha_{T} \sin \beta_{T} \\
-\frac{1}{V_{T}} \sec \beta_{T} \sin \alpha_{T} & 0 & \frac{1}{V_{T}} \cos \alpha_{T} \sec \beta_{T}
\end{array}\right]
$$

The scalar forms of the translational dynamics equations are presented in appendix B.

\subsubsection{Rotational kinematics equations}

The rotational kinematics equation in matrix form is the well known standard equation:

$$
\mathbf{R}_{\mathbf{B}_{\mathbf{T}} \mathbf{I}} \dot{\mathbf{R}}_{\mathbf{B}_{\mathbf{T}} \mathbf{I}}=-\mathbf{S}\left(\omega_{\mathbf{B}_{\mathbf{T}}}\right)
$$

where $\omega_{\mathbf{B}_{\mathbf{T}}}$ is the representation of the angular velocity vector of the tanker relative to the inertial frame expressed in its own body frame as

$$
\omega_{\mathbf{B}_{\mathbf{T}}}=\left[\begin{array}{c}
p_{T} \\
q_{T} \\
r_{T}
\end{array}\right]
$$

\subsubsection{Rotational dynamics equations}

The matrix form of the rotational dynamics of the tanker is modeled with the standard rotational dynamics equation.

$$
\dot{\omega}_{B_{T}}=\underline{\underline{\mathbf{I}}}_{\mathbf{T}}^{-\mathbf{1}} M_{B_{T}}+\underline{\underline{\mathbf{I}}}_{\mathbf{T}}^{-\mathbf{1}} \mathbf{S}\left(\omega_{\mathbf{B}_{\mathbf{T}}}\right) \underline{\underline{\mathbf{I}}}_{\mathbf{T}} \omega_{B_{T}}
$$


where $\underline{\mathbf{I}}_{\mathbf{T}}$ is the inertia matrix of the tanker aircraft, $M_{B_{T}}$ is the moment of the external forces around the origin of tanker body frame and expressed in the tanker body frame as

$$
M_{B_{T}}=\left[\begin{array}{c}
\mathcal{L}_{\mathcal{T}} \\
\mathcal{M}_{\mathcal{T}} \\
\mathcal{N}_{\mathcal{T}}
\end{array}\right]
$$

\subsubsection{Engine dynamics}

The thrust generated by the engine $\left(T_{T}\right)$ is

$$
T_{T}=\xi_{T} T_{\max _{T}}
$$

where $\xi_{T}$ denotes the instantaneous throttle setting and $T_{\max _{T}}$ is the maximum available thrust of the tanker and assumed to be constant. The engine dynamics is modeled as that of a first order system with time constant $\tau_{T}$. Therefore, we have

$$
\dot{\xi}_{T}=\frac{\xi_{T}-\xi_{t_{T}}}{\tau_{T}}
$$

where $\xi_{t_{T}}$ is the commanded throttle setting $\left(0 \leq \xi_{t_{T}} \leq 1\right)$.

\subsubsection{Actuator dynamics}

For the present study, only the actuator saturations are considered. The deflection range attainable from each control surface is (-20 deg, $20 \mathrm{deg}$ )

\subsubsection{Tanker controller}

The tanker aircraft flies at a constant altitude with a constant airspeed. In general, it flies in racetrack orbit pattern waiting for the receiver aircraft for rendezvous or for air refueling if the refueling area is restricted. Thus, the tanker's controller is 
implemented to fly at any commanded altitude and with any commanded speed and commanded steady turn by the commanded yaw rate. It should track commanded yaw rate changes with small transient and zero steady-state error. While starting and ending a turn, and during the turn, deviations in altitude and speed from their respective nominal values should be small and decay to zero at the steady-state.

A combination of a multi-input-multi-output state feedback LQR and integral control technique is employed for the altitude and speed hold, and yaw rate tracking controller. The control variables available for the tanker aircraft are the three conventional control surfaces and the throttle setting. The outputs to be controlled are the airspeed, altitude and yaw rate. The overall structure of the closed loop system is shown in Fig. 2.2.

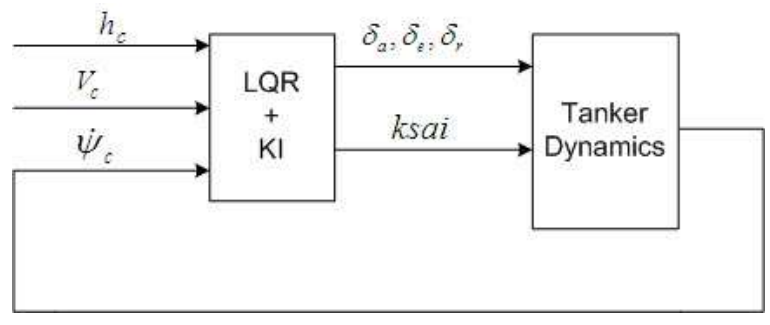

Figure 2.2. The tanker's control diagram.

A gain scheduling scheme is implemented based on the commanded speed and yaw rate. The tanker's equations of motion are linearized at four nominal conditions $\left(\dot{\psi}_{T}, V_{T}\right)=(0 \mathrm{deg} / \mathrm{s}, 180 \mathrm{~m} / \mathrm{s}),(0 \mathrm{deg} / \mathrm{s}, 200 \mathrm{~m} / \mathrm{s}),(1.7 \mathrm{deg} / \mathrm{s}, 180 \mathrm{~m} / \mathrm{s}),(1.7 \mathrm{deg} / \mathrm{s}$, $200 \mathrm{~m} / \mathrm{s}$ ). Lagrange interpolation scheme is utilized to formulate the overall non-linear controller based on the linear designs at the four nominal conditions above. Note also that the control law assumes the availability of full state measurement or estimation for feedback. 
2.5 Receiver aircraft model and controller

The full dynamic model of the receiver aircraft derived in a previous work by [16] and the controller designed and validated by [17] are detailed in this section.

The receiver aircraft needs to be controlled with respect to the tanker's position and orientation rather than with respect to the inertial frame. Moreover, the receiver aircraft will be exposed to a nonuniform wind field when it is in the proximity of the tanker due to the trailing vortex of the tanker.

\subsubsection{Translational kinematics equations}

The translational kinematics equation is written in terms of the position vector of the receiver with respect to the tanker, not its absolute position vector with respect to the inertial frame. In matrix form,

$$
\dot{\xi}=\mathbf{R}_{\mathbf{B}_{\mathbf{R}} \mathbf{B}_{\mathbf{T}}}^{\mathbf{T}} \mathbf{R}_{\mathbf{B}_{\mathbf{R}} \mathbf{w}_{\mathbf{R}}} V_{w}+\mathbf{R}_{\mathbf{B}_{\mathbf{R}} \mathbf{B}_{\mathbf{T}}}^{\mathbf{T}} W-\mathbf{R}_{\mathbf{B}_{\mathbf{T}} \mathbf{I}} \dot{r}_{B_{T}}+\mathbf{S}\left(\omega_{\mathbf{B}_{\mathbf{T}}}\right) \xi
$$

where $\xi$ is the position of the receiver relative to the tanker expressed in the body frame of the tanker, $\mathbf{R}_{\mathbf{B}_{\mathbf{R}} \mathbf{w}_{\mathbf{R}}}$ is the rotation matrix from the receiver wind frame to body frame, $V_{w}$ is the velocity of the receiver relative to the surrounding air expressed in the receiver wind frame, $W$ is the velocity of the surrounding air relative to the ground expressed in the receiver body frame, $\mathbf{R}_{\mathbf{B}_{\mathbf{R}} \mathbf{B}_{\mathbf{T}}}$ is the rotation matrix from tanker body frame to receiver body frame, and $\dot{r}_{B_{T}}$ is the velocity of the tanker relative to the inertial frame. The scalar forms of Eq. (2.10) without the wind terms are presented in appendix $\mathrm{C}$. 
2.5.2 Translational dynamics equations

The translational dynamics equation of the receiver aircraft including the wind effect in matrix form is [11]

$$
\begin{aligned}
{\left[\begin{array}{c}
\dot{V}_{R} \\
\dot{\beta}_{R} \\
\dot{\alpha}_{R}
\end{array}\right]=} & \mathcal{E}_{\mathbf{R}}^{-\mathbf{1}}\left[\mathbf{S}\left(\omega_{\mathbf{B}_{\mathbf{R}} \mathbf{B}_{\mathbf{T}}}\right)+\mathbf{R}_{\mathbf{B}_{\mathbf{R}} \mathbf{B}_{\mathbf{T}}} \mathbf{S}\left(\omega_{\mathbf{B}_{\mathbf{T}}}\right) \mathbf{R}_{\mathbf{B}_{\mathbf{R}} \mathbf{B}_{\mathbf{T}}}^{\mathbf{T}}\right]\left(\mathbf{R}_{\mathbf{B}_{\mathbf{R}} \mathbf{w}_{\mathbf{R}}} V_{w}+W\right) \\
& -\mathcal{E}_{\mathbf{R}}^{-\mathbf{1}} \dot{W}+\frac{1}{m_{R}} \mathcal{E}_{\mathbf{R}}^{-\mathbf{1}}\left(\mathbf{R}_{\mathbf{B}_{\mathbf{R}} \mathbf{B}_{\mathbf{T}}} \mathbf{R}_{\mathbf{B}_{\mathbf{T}} \mathbf{I}} M_{R}+\mathbf{R}_{\mathbf{B}_{\mathbf{R}} \mathbf{w}_{\mathbf{R}}} A_{R}+P_{R}\right)
\end{aligned}
$$

where

$$
\mathcal{E}_{\mathbf{R}}^{-1}=\left[\begin{array}{ccc}
\cos \alpha \cos \beta & \sin \beta & \cos \beta \sin \alpha \\
-\frac{1}{V_{R}} \cos \alpha \sin \beta & \frac{1}{V_{R}} \cos \beta & -\frac{1}{V_{R}} \sin \alpha \sin \beta \\
-\frac{1}{V_{R}} \sec \beta \sin \alpha & 0 & \frac{1}{V_{R}} \cos \alpha \sec \beta
\end{array}\right]
$$

The scalar forms of Eq. (2.11) are presented in appendix C.

\subsubsection{Rotational kinematics equations}

The rotational motion of the receiver aircraft, similar to its translational motion, is also analyzed with reference to the tanker body frame. Even though the standard rotational kinematics and dynamics equations are used, their interpretations are different because both angular position and angular velocity of the receiver aircraft are relative to the tanker body frame, an accelerating and rotating reference frame.

The rotational kinematics equation of the receiver aircraft in matrix form is also the well known standard equation:

$$
\mathbf{R}_{\mathbf{B}_{\mathbf{R}} \mathbf{B}_{\mathbf{T}}} \dot{\mathbf{R}}_{\mathbf{B}_{\mathbf{R}} \mathbf{B}_{\mathbf{T}}}^{\mathbf{T}}=-\mathbf{S}\left(\omega_{\mathbf{B}_{\mathbf{R}} \mathbf{B}_{\mathbf{T}}}\right)
$$


where $\omega_{B_{R} B_{T}}$ is the representation of the angular velocity vector of the receiver aircraft relative to the tanker body frame expressed in its own body frame as

$$
\omega_{B_{R} B_{T}}=\left[\begin{array}{c}
p_{R_{T}} \\
q_{R_{T}} \\
r_{R_{T}}
\end{array}\right]
$$

The scalar forms of this matrix equation in terms of Euler angles are represented in Appendix C.

\subsubsection{Rotation dynamics equations}

The matrix form of the rotational dynamics of the receiver is also modeled as

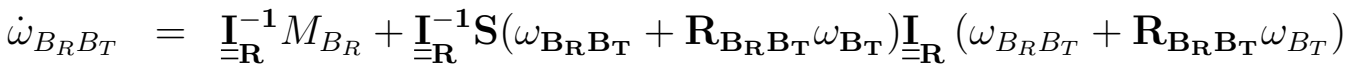

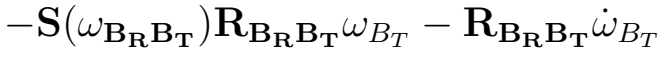

where $\underline{\underline{\mathbf{I}}}_{\mathbf{R}}$ is the inertia matrix of the receiver aircraft, $M_{B_{R}}$ is the moment of the external forces around the origin of the receiver body frame and expressed in the receiver body frame as

$$
M_{B_{R}}=\left[\begin{array}{c}
\mathcal{L} \\
\mathcal{M} \\
\mathcal{N}
\end{array}\right]
$$

\subsubsection{Engine dynamics}

As in the case of the tanker, the engine model of the receiver is also a first order transfer function with constant maximum thrust, obviously with different maximum thrust and different time constant. 


\subsubsection{Actuator dynamics}

For the present study, only the actuator saturation and rate limit effects are considered for the receiver. The deflection range attainable from the aileron is $(-30$ deg,30 deg), from the elevator (-30 deg,30 deg) and from the rudder (-60 deg,60 deg). All three control effectors have a rate limit of $\pm 90 \mathrm{deg} / \mathrm{sec}$.

\subsubsection{Receiver controller}

The receiver controller is a trajectory-tracking controller which makes the receiver follow the reference trajectory in a safe and timely manner or to track the generated trajectories, with zero steady-state error in the $x, y, z$ coordinates in the tanker's body frame, under the disturbance of trailing vortex, time variation of the inertia properties of the receiver and the possible steady maneuvers of the tanker's body frame. Meanwhile,the control inputs generated by the controller should not cause significant saturation on the magnitudes and rates of the actuators. Moreover, during the transient,overshoot or undershoot on trajectory response should be minimized to ensure the safety. At the same time, the response of the closed loop system should be fast enough so that the approach and fly-away and station-keeping maneuvers are completed as planned and high-wind regions of the trailing vortex field are exited in a timely fashion. A very big pitch angle should not be commanded. Finally, to ensure the safety of the aircraft, the bank angle should be small relative to its nominal value. The over all structure of the closed loop system in Fig. 2.3.

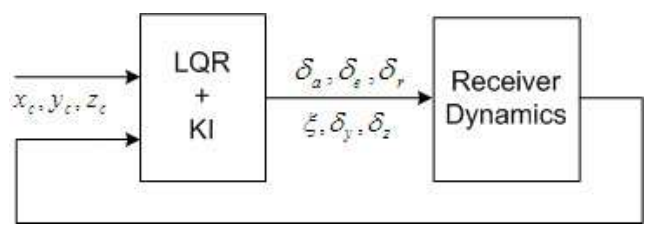

Figure 2.3. The receiver's control diagram. 
Similar to the tanker aircraft, a combination of multi-input-multi-output statefeedback LQR and integral control technique is employed in designing the position tracking controller. Additionally, a gain scheduling scheme is implemented based on the speed and turn rate. The equations of motion are linearized in four nominal conditions, similar to the tanker nominal conditions as $\left(\dot{\psi}_{T}, V_{T}\right)=(0 \mathrm{deg} / \mathrm{s}, 180 \mathrm{~m} / \mathrm{s})$, $(0 \mathrm{deg} / \mathrm{s}, 200 \mathrm{~m} / \mathrm{s}),(1.7 \mathrm{deg} / \mathrm{s}, 180 \mathrm{~m} / \mathrm{s}),(1.7 \mathrm{deg} / \mathrm{s}, 200 \mathrm{~m} / \mathrm{s})$, Lagrange interpolation scheme is utilized. Note that the control law assumes the availability of full state measurement of estimation for feedback. 


\section{CHAPTER 3}

\section{GUIDANCE ALGORITHMS}

\subsection{Introduction}

This chapter describes the guidance algorithms for the tanker and the receiver aircraft to rendezvous in the presence of wind. While the focus of this work is to develop a guidance algorithm for the receiver aircraft to successfully rendezvous with the tanker aircraft, the evaluation of the receiver guidance algorithm in the integrated simulation environment requires the guidance of the tanker as well. For a successful rendezvous, the tanker is required to fly on the initially fixed refueling orbit. The receiver aircraft is responsible for meeting the tanker aircraft at the geographically fixed location of the ARCP.

\subsection{Tanker aircraft}

In the modified point-parallel rendezvous procedure, the tanker aircraft is required to fly in a racetrack pattern that is geographically fixed. However, the current controller of the tanker, as described in section 2.4.7, is not readily suited for this task in the presence of wind. The controller is designed to track commanded altitude, airspeed and yaw rate. In the absence of wind, commanded airspeed and yaw rate can easily result in a perfect geographically fixed racetrack pattern. However, the presence of horizontal prevailing wind will distort the racetrack pattern relative to the inertial frame.

This difficulty is overcome by the employment of a virtual target that is free from wind effect and thus can fly in a geographically-fixed racetrack pattern. Then, 
the motion of the virtual target is used to generate the commanded signals, specifically the yaw rate commands for the current controller of the tanker.

\subsubsection{Virtual target trajectory}

For the virtual target trajectory, the standard translational kinematics in terms of heading and flight path angle is used.

$$
\begin{aligned}
\dot{x} & =V_{V} \cos \gamma \cos \mu \\
\dot{y} & =V_{V} \cos \gamma \sin \mu \\
\dot{z} & =V_{V} \sin \gamma
\end{aligned}
$$

where $V_{V}$ is the virtual target speed, $\gamma$ is flight path angle and $\mu$ is the heading angle. The position of the virtual target $(x, y, z)$ relative to the inertial frame is controlled by the speed, heading and flight path angles. For a racetrack trajectory at constant altitude and constant speed along the track, the following is set for speed and flight path angle

$$
\begin{aligned}
V_{V} & =V_{T} \\
\gamma & =0
\end{aligned}
$$

where $V_{T}$ is the specified speed of the tanker flying on the refueling orbit.

To fly the virtual target on the refueling orbit, the required heading angle should be commanded. Obviously, on the straight legs of the racetrack orbit, the heading should be constant. During the turns, constant heading rate, $\dot{\mu}$, is used. To generate any aerial refueling orbit, the required $\dot{\mu}$ for a given lateral offset $\left(h_{o s}\right)$ should be calculated. The following discussion gives the details of deriving an analytical expression for $\dot{\mu}$ as a function of $h_{o s}$ and total heading change $(\triangle \mu)$ after the turn. Note that for standard racetrack maneuvers, $\triangle \mu$ should be 180 degrees. However, 


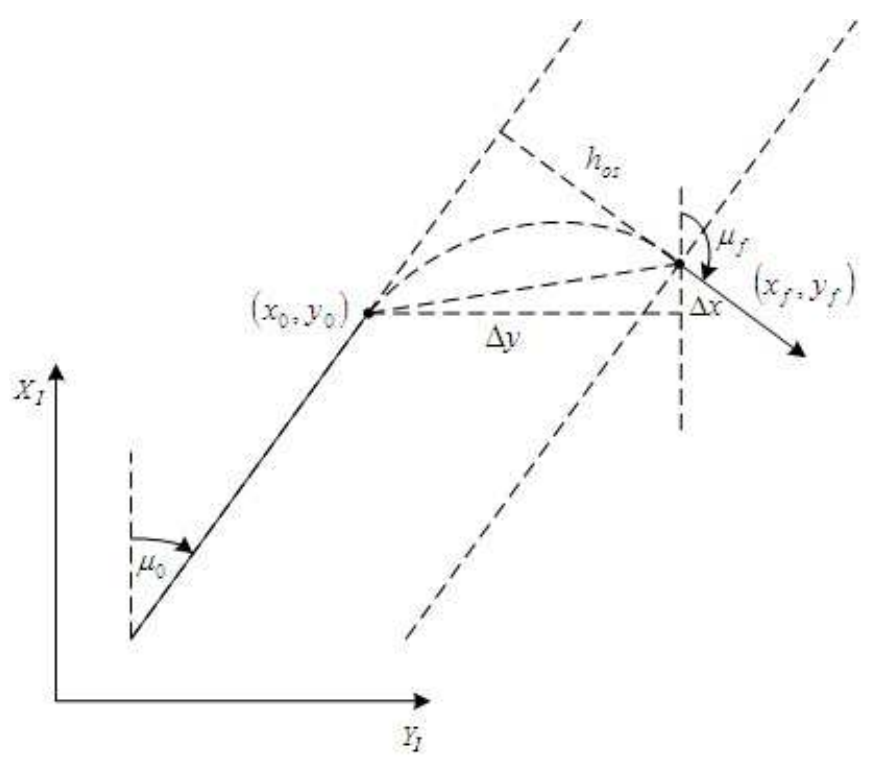

Figure 3.1. Diagram of virtual target trajectory.

the analytic expression is derived for an arbitrary $\triangle \mu$. As depicted in Fig. 3.1, the heading angle is $\mu_{0}$ before the turn and should be $\mu_{f}$ after the turn. The turn should result in a lateral offset of $h_{o s}$. The question then is what $\dot{\mu}$ will yield $\triangle \mu=\mu_{f}-\mu_{0}$ heading change and $h_{o s}$ lateral offset.

The translational kinematics in Eqs. (3.1)-(3.3), when the flight path angle $\gamma=0$ and $V_{V}=V_{T}$ constant, implies

$$
\begin{aligned}
& \dot{x}=V_{T} \cos \mu \\
& \dot{y}=V_{T} \sin \mu
\end{aligned}
$$

which implies

$$
\begin{gathered}
\triangle x=\frac{V_{T}}{\dot{\mu}}\left(\sin \mu-\sin \mu_{0}\right) \\
\triangle y=-\frac{V_{T}}{\dot{\mu}}\left(\cos \mu-\cos \mu_{0}\right)
\end{gathered}
$$

From Fig. 3.1, it can be shown that 


$$
h_{o s}=\triangle y \cos \mu_{0}-\triangle x \sin \mu_{0}
$$

Substituting $\triangle x$ and $\triangle y$ yields

$$
\begin{aligned}
h_{o s}= & -\frac{V_{T}}{\dot{\mu}}\left(\cos \mu \cos \mu_{0}-\cos ^{2} \mu_{0}\right) \\
& -\frac{V_{T}}{\dot{\mu}}\left(\sin \mu \sin \mu_{0}-\sin ^{2} \mu_{0}\right) \\
= & -\frac{V_{T}}{\dot{\mu}}\left(\cos \mu \cos \mu_{0}+\sin \mu \sin \mu_{0}\right)+\frac{V_{T}}{\dot{\mu}} \\
= & \frac{V_{T}}{\dot{\mu}}(1-\cos \triangle \mu)
\end{aligned}
$$

which implies

$$
\dot{\mu}=\frac{V_{T}}{h_{o s}}(1-\cos \triangle \mu)
$$

where $h_{o s}$ should be positive for right turn and negative for left turn. Using $\dot{\mu}=0$ for straight legs and $\dot{\mu}$ from Eq. (3.6) for turns generate any aerial refueling orbit. The turns are triggered based on a simulation-based schedule.

\subsubsection{Nonlinear guidance logic for virtual target tracking}

The virtual target flies on the aerial refueling orbit with speed $V_{T}$ at the refueling altitude. The heading rate is $\dot{\mu}$ is adjusted to generate the straight legs and turns of the orbit. The translational kinematics in Eq. (3.1) to Eq. (3.3) produces the $(x, y, z)$ trajectory in the inertial frame. As the virtual target moves on the refueling orbit, a nonlinear guidance algorithm [1] is utilized for the tanker to follow the virtual target. This should result in the tanker flying on the very same refueling orbit. This guidance logic computes lateral acceleration commands to minimize the lateral error between the tanker and the virtual target flying on the refueling orbit. The lateral acceleration commands are converted to the yaw rate commands for the tanker's controller. This nonlinear guidance logic is designed for guiding unmanned 
air vehicles (UAVs) on curved trajectories. It uses inertial speed in the computation of commanded lateral acceleration and adds adaptive capability to the change of vehicle speed due to external disturbances such as wind. This guidance logic is used for air rendezvous of the two aircraft [1]. Based on the benefits of this nonlinear guidance logic stated by the author in [1], this thesis work applies it to rendezvous of the tanker and receiver aircraft. Fig. 3.2 depicts the guidance algorithms and related variables. The first step for the implementation of the guidance logic is to select a reference

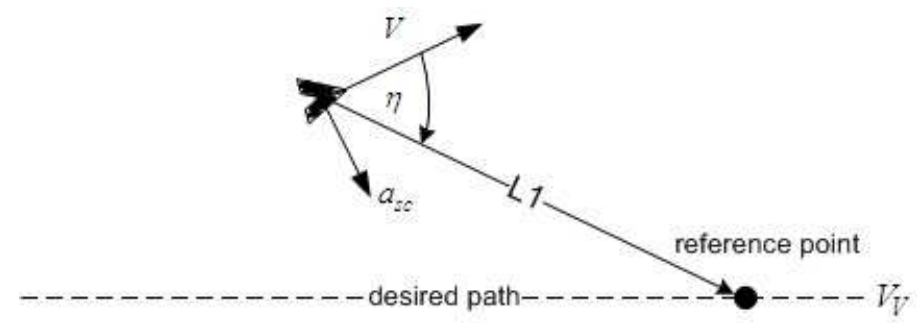

Figure 3.2. Diagram of nonlinear guidance logic.

point on the curved trajectory to follow. In the case of this research, the reference point is the position of the virtual target moving on the refueling orbit. Based on the relative position between the virtual target position and the tanker position (see Fig. 3.2) the acceleration command is calculated as

$$
a_{s c}=2 \frac{V^{2}}{L_{1}} \sin \eta
$$

where $L_{1}$ is line defined from vehicle position to a reference point on the desired trajectory, $V$ is vehicle velocity, $\eta$ is an angle from $V$ to the line $L_{1}$ (clockwise direction is positive) and $a_{s c}$ is the lateral acceleration command. There are two significant properties of the guidance equation: (i) The direction of the acceleration depends on the sign of the angle between the $L_{1}$ line segment and the vehicle velocity vector. (ii) At each point in time a circular path can be defined by the position of the 
reference point, the vehicle position, and tangential to the vehicle velocity vector. The acceleration command is equal to the centripetal acceleration required to follow this instantaneous circular segment. Hence the guidance logic will produce a lateral acceleration that is appropriate to follow a circle of any radius.

Since the low-level controller of the tanker takes yaw-rate command, the acceleration command should be converted to yaw-rate command. First, the sideways acceleration command can be converted to bank angle command as [1]

$$
\phi_{c}=\frac{a_{s c}}{g}
$$

To convert the bank angle command to yaw rate command, the rotational kinematics of the steady coordinated turn is utilized as

$$
\begin{aligned}
\dot{\psi}_{0} & =q_{0} \sin \phi_{0}+r_{0} \cos \phi_{0} \\
0 & =q_{0} \cos \phi_{0}-r_{0} \sin \phi_{0}
\end{aligned}
$$

which imply

$$
\begin{aligned}
& r_{0}=\dot{\psi}_{0} \cos \phi_{0} \\
& q_{0}=\dot{\psi}_{0} \sin \phi_{0}
\end{aligned}
$$

Additionally, the requirement of no lateral aerodynamic/propulsion force implies

$$
-m r_{0} u_{0}+m g \sin \phi_{0}=0
$$

Substituting $r_{0}$ from Eq. (3.12 in the equation and rearranging yields

$$
\dot{\psi}_{0}=\frac{g}{u_{0}} \tan \phi_{0}
$$

Substituting $\phi_{c}$ from Eq. (3.8) in this equation yields the expression for yaw-rate command based on acceleration command as

$$
\dot{\psi}_{c}=\frac{g}{u_{0}} \tan \left(\frac{a_{s c}}{g}\right)
$$


Fig. 3.3 illustrates the relation between the virtual target position and the yaw-rate command for the tanker.

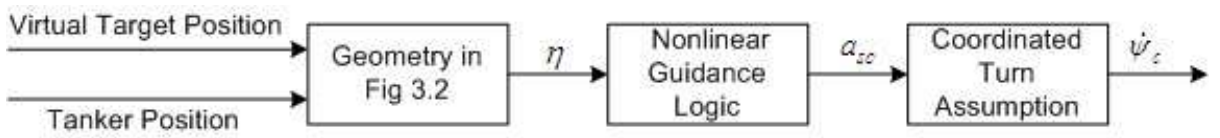

Figure 3.3. The relationship of lateral acceleration and commanded yaw rate.

\subsubsection{Proportional speed control for tanker aircraft}

The virtual target moves with the specified speed relative to the inertial frame and is not affected by the presence of the wind. However, the tanker is exposed to the prevailing wind, which increases the inertial speed in the case of tail wind and decreases it in the case of headwind. This is when the constant airspeed is commanded (Recall that the speed controller of the tanker controls the airspeed, not the inertial speed). The change in inertial speed due to the prevailing wind causes the tanker to move faster or slower than the virtual target. This results in the tanker overtaking or falling behind the virtual target, which causes problem in the application of the nonlinear guidance logic. To decrease the variation of the distance between the tanker and the virtual target, a proportional controller is employed for the calculation of the commanded airspeed of the tanker as

$$
V_{c}=190+K_{p}(|\Delta x|-800)
$$

where $K_{p}$ is the proportional gain and $\Delta x$ is the distance along $x$ axis between the tanker aircraft and the virtual target aircraft. 


\subsection{Receiver aircraft}

As explained in section 2.5.8, the receiver aircraft has a trajectory tracking controller. This controller is designed to keep the receiver aircraft at a position or along a trajectory relative to the tanker aircraft body frame, which is moving and rotating reference frame. This controller is capable of keeping the receiver at the pre-contact position, maneuvering it from the pre-contract position to the contact position and keeping it there for the refueling while the tanker is flying on a racetrack maneuver. During the rendezvous phase, the receiver aircraft should fly along a straight line from ARIP to ARCP, as depicted in Fig. 1.1. While flying along the straight line, the receiver should synchronize its motion with the tanker flying on the refueling orbit such that it meets the tanker aircraft at ARCP. When at ARCP, the receiver should initiate the formation with the tanker by positioning itself at the pre-contract position relative to the tanker aircraft.

The synchronization of the receiver with the tanker motion is done through the concept of the virtual tanker. The distance-to-travel by the tanker along the refueling orbit to the AP (Anchor Point), as depicted in Fig. 1.1, is calculated. The virtual tanker is placed along the straight line from ARIP to ARCP or AP. The receiver controller is commanded to keep the receiver aircraft at the pre-contract position relative to the virtual tanker. As the actual tanker flies closer to AP along the refueling orbit, the virtual tanker flies towards AP along the straight line by maintaining the same distance-to-travel. Since the receiver is commanded to maintain its relative position with respect to the virtual tanker, the receiver also travels along the straight line towards ARCP and AP. By the synchronization, the virtual target is guaranteed to arrive at $\mathrm{ARCP}$ at the same time as the virtual tanker. Then, the receiver is commanded to switch from the virtual tanker to the actual tanker. Since the receiver is at the pre-contract position relative to the virtual tanker and the virtual tanker 
and actual tanker meet at $\mathrm{ARCP}$, the receiver aircraft is automatically placed at the pre-contract position relative to the actual tanker after the switch.

This method of rendezvous would work perfectly in the absence of wind. However, in the case of prevailing wind, the tanker and the receiver both cannot track the required trajectories perfectly. The details of this method and the approaches taken to dealing with the deviation of the aircraft from their corresponding trajectories are explained below in this section.

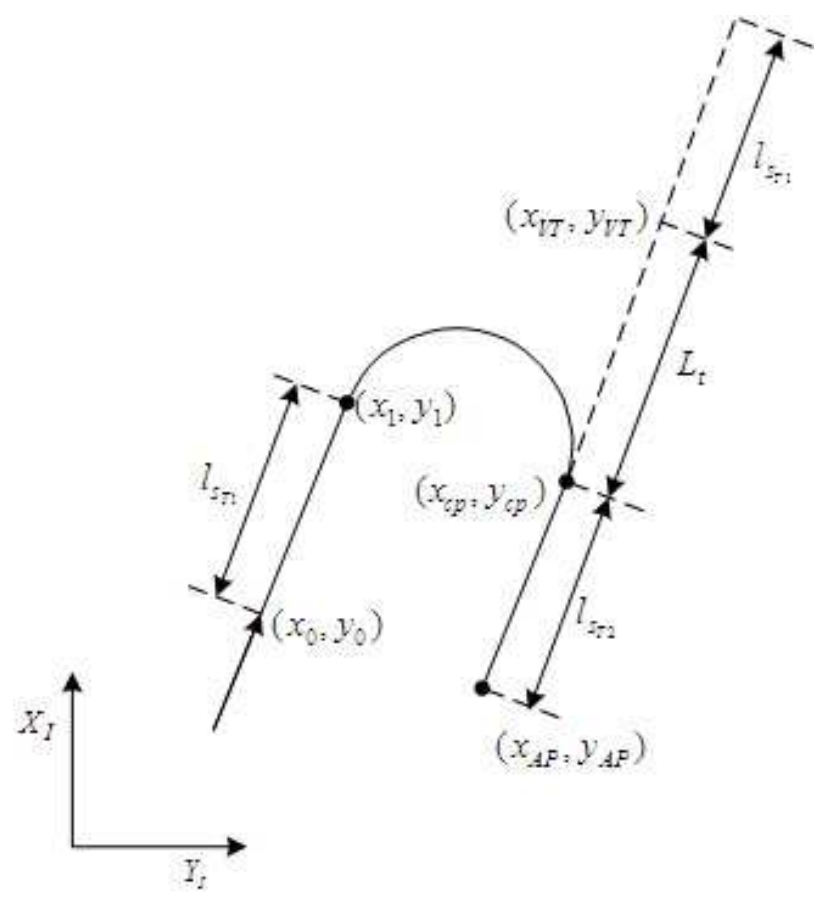

Figure 3.4. Diagram of the virtual tanker aircraft trajectory.

Fig. 3.4 shows the depiction of the problem. The position of the virtual target that the tanker follows is $\left(x_{0}, y_{0}\right)$. The position at which the virtual target starts turning is $\left(x_{1}, y_{1}\right)$. The position where the virtual target stops its turn and starts the straight leg towards the Anchor Point is $\left(x_{C P}, y_{C P}\right)$, which is ARCP. 
The distance-to-travel to AP for the virtual target is the sum of the lengths of the straight legs and the semi-circle from $\left(x_{1}, y_{1}\right)$ to $\left(x_{C P}, y_{C P}\right)$. The lengths of the straight legs are

$$
l_{s_{T 1}}= \begin{cases}\sqrt{\left(x_{1}-x_{0}\right)^{2}+\left(y_{1}-y_{0}\right)^{2}} & , \text { before the turn starts } \\ 0 & \text {,once the turn starts }\end{cases}
$$

and

$$
l_{s_{T 2}}= \begin{cases}0 & \text {,before the turn completes } \\ \sqrt{\left(x_{C P}-x_{0}\right)^{2}+\left(y_{C P}-y_{0}\right)^{2}} & \text {,after the turn }\end{cases}
$$

The length of the semicircle is calculated based on the fact that the diameter of the circle is the lateral offset between the two straight legs of the refueling orbit, $h_{o s}$.

$$
\left(x_{1}-x_{C P}\right)^{2}+\left(y_{1}-y_{C P}\right)^{2}=h_{o s}^{2}
$$

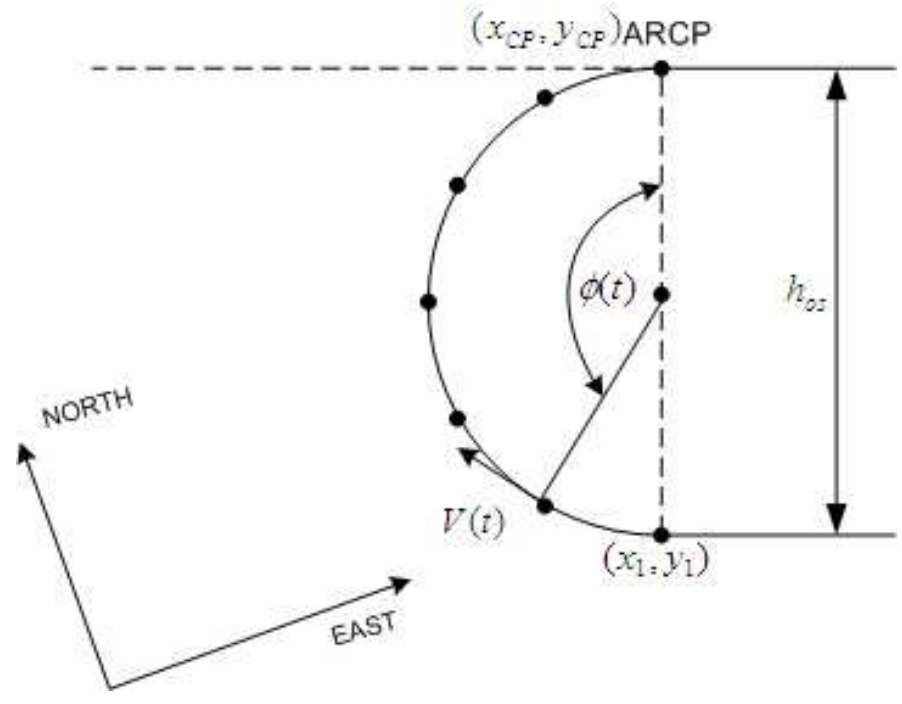

Figure 3.5. Diagram in the rendezvous turn. 
When the virtual target is turning, the arc length to travel to reach ARCP at $\left(x_{C P}, y_{C P}\right)$ can be calculated as

$$
L(t)=\frac{h_{o s}}{2} \phi(t)
$$

As the virtual tanker travels on the circle, $\phi(t)$ goes from $\pi$ (which is $\left(x_{1}, y_{1}\right)$ ) to 0 (which is $\left.\left(x_{C P}, y_{C P}\right)\right)$, as depicted in Fig. 3.5. For the calculation of $L(t), \phi(t)$

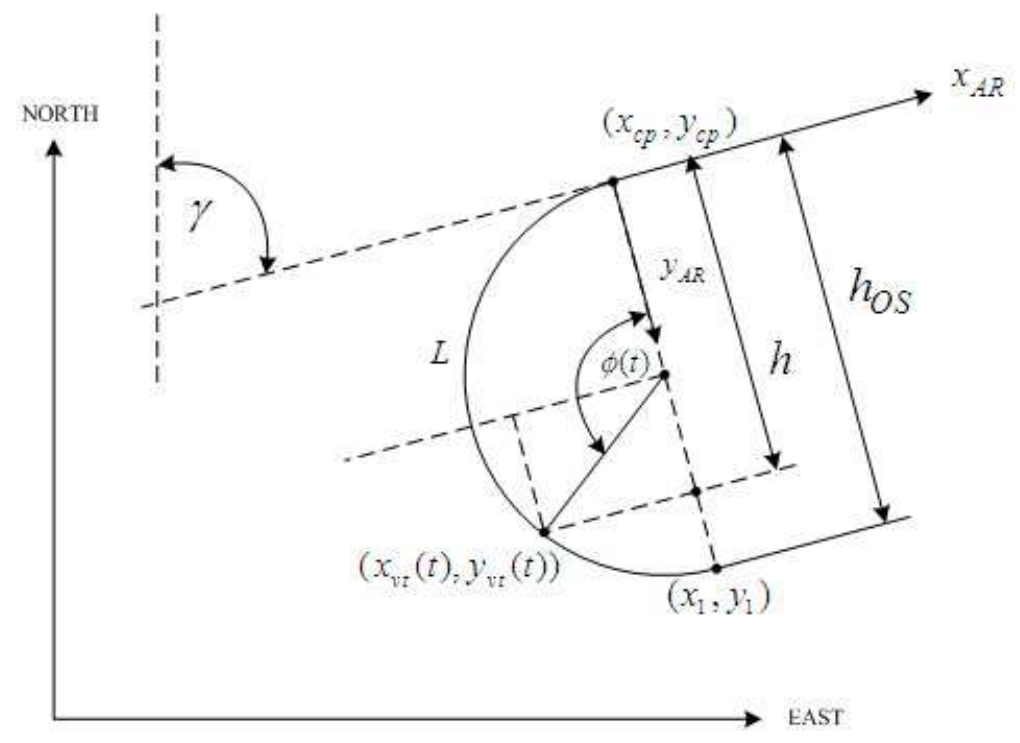

Figure 3.6. Diagram in the rendezvous turn.

should be computed for given virtual target position on the turn arc. For this, a new reference frame, called "Aerial Refueling (AR) Frame", is introduced as shown in Fig. 3.6. Angle $\gamma$ in this context is to define the orientation of AR frame with respect to the inertial frame, i.e., angle $\gamma$ is the angle between the $x$-axis of AR frame and the $x$-axis of the inertial frame. Note that the origin of AR frame is at ARCP, $\left(x_{C P}, y_{C P}\right)$, defined in the inertial frame.

In AR frame, the ARCP is located at $(0,0)$ and $\left(x_{1}, y_{1}\right)$ is located at $\left(0, h_{o s}\right)$. Let $\left(x_{v t, A R}, y_{v t, A R}\right)$ be the position of the virtual tanker with respect to the origin of 


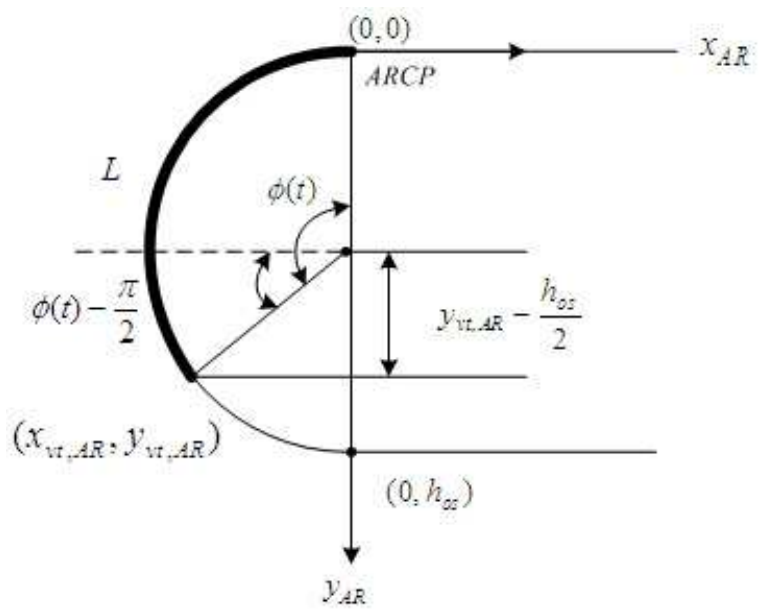

Figure 3.7. Diagram in the rendezvous.

the AR frame, expressed in the AR frame. Note that as $\phi$ goes from $\pi$ to $0, y_{v t, A R}$ goes from $h_{o s}$ to 0 and $y_{v t, A R}-\frac{h_{o s}}{2}$ goes from $\frac{h_{o s}}{2}$ to $-\frac{h_{o s}}{2}$, as depicted in Fig. 3.7. Note from Fig. 3.7 that

$$
\sin \left(\phi-\frac{\pi}{2}\right)=\frac{y_{v t, A R}-\frac{h_{o s}}{2}}{\frac{h_{o s}}{2}}
$$

which implies that

$$
\cos \phi=1-\frac{2 y_{v t, A R}}{h_{o s}}
$$

For the implementation of this equation, $y_{v t, A R}$ should be computed from $\left(x_{v t}, y_{v t}\right)$, the position of the virtual tanker in the inertial frame.

$$
\left[\begin{array}{c}
x_{v t} \\
y_{v t}
\end{array}\right]=\left[\begin{array}{l}
x_{C P} \\
y_{C P}
\end{array}\right]+R^{T}\left[\begin{array}{l}
x_{v t, A R} \\
y_{v t, A R}
\end{array}\right]
$$

where

$$
R=\left[\begin{array}{cc}
\cos \gamma & \sin \gamma \\
-\sin \gamma & \cos \gamma
\end{array}\right]
$$


which is the 2-D rotation matrix from inertial frame to AR frame. Rearranging Eq. (3.24) yields, in scalar form,

$$
\begin{gathered}
x_{v t, A R}=\left(x_{v t}-x_{C P}\right) \cos \gamma+\left(y_{v t}-y_{C P}\right) \sin \gamma \\
y_{v t, A R}=-\left(x_{v t}-x_{C P}\right) \sin \gamma+\left(y_{v t}-y_{C P}\right) \cos \gamma
\end{gathered}
$$

Recall that the objective is to place the virtual tanker along the aerial refueling line (straight line passing through ARIP and ARCP) for the receiver to follow until switching to the actual tanker after ARCP. Note that the aerial refueling line is along the $x$-axis of the AR frame. Thus, the point on the aerial refueling line that has the same distance-to-travel to ARCP is

$$
\left[\begin{array}{l}
x_{v t i} \\
y_{v t i}
\end{array}\right]=\left[\begin{array}{l}
x_{C P} \\
y_{C P}
\end{array}\right]+R^{T}\left[\begin{array}{c}
-\left(L+l_{s t}\right) \\
0
\end{array}\right]
$$

which is named "image of the virtual target" on the aerial refueling line (See Fig. 3.7). $R$ in Eq. (3.28) is the same rotation matrix defined earlier in Eq. (3.25). Eq. (3.25), in scalar form, gives

$$
\begin{aligned}
& x_{v t i}=x_{C P}-\left(l_{s_{T 1}}+L\right) \cos \gamma \\
& y_{v t i}=y_{C P}-\left(l_{s_{T 1}}+L\right) \sin \gamma
\end{aligned}
$$

This equation is used to calculated the position of the image of the virtual target along the aerial refueling line while the virtual target is on the first straight leg or the turn arc of the refueling orbit. Before the turn, $L$ is 0 . Once the virtual target starts turning, $l$ is set to 0 in the equation. Note that once the virtual target completes the turn at ARCP, it is on the aerial refueling line. Thus, no need to calculate the position of its image on the aerial refueling line. In other words, 


$$
\begin{aligned}
& x_{v t i}=x_{v t} \\
& y_{v t i}=y_{v t}
\end{aligned}
$$

Recall that the nonlinear guidance logic for the tanker is designed to follow the virtual target from a distance, $L_{1}$. Further, due to the prevailing wind, the tanker may deviate from the refueling orbit, which the virtual target follows perfectly. Therefore, the virtual tanker is not placed at the image of the virtual target, as calculated in Eqs. (3.29) and (3.30). Instead, the position of the actual tanker with respect to the virtual target is formulated. This relative position is added to the image of the virtual target on the aerial refueling line to calculate the position of the virtual tanker that the receiver is to follow.

As shown in Fig. 3.8, the relative position vector between the virtual target and the tanker is calculated based on vector summation.

$$
\underline{\mathrm{r}}_{T}+\triangle \underline{\mathrm{r}}=\underline{\mathrm{r}}_{v t}
$$

where $\underline{\underline{r}}_{T}$ is the position of the tanker and $\underline{r}_{v t}$ is the position of the virtual target. Eq. (3.33) implies

$$
\triangle \underline{\mathrm{r}}=\underline{\mathrm{r}}_{v t}-\underline{\mathrm{r}}_{T}
$$

This relative position vector will be used to calculate the position of the virtual tanker based on the position of the image of the virtual target.

Similar to Eq. (3.33), the relation between the position vectors of the virtual target image and the virtual tanker is (See Fig. 3.7)

$$
\underline{\mathrm{r}}_{V T}+\triangle \underline{\mathrm{r}}=\underline{\mathrm{r}}_{v t i}
$$

where $\underline{\mathrm{r}}_{V T}$ is the position of the virtual tanker and $\underline{\mathrm{r}}_{v t i}$ is the position of the image of the virtual target. Eq. (3.35) is solved for the position of the virtual tanker as 


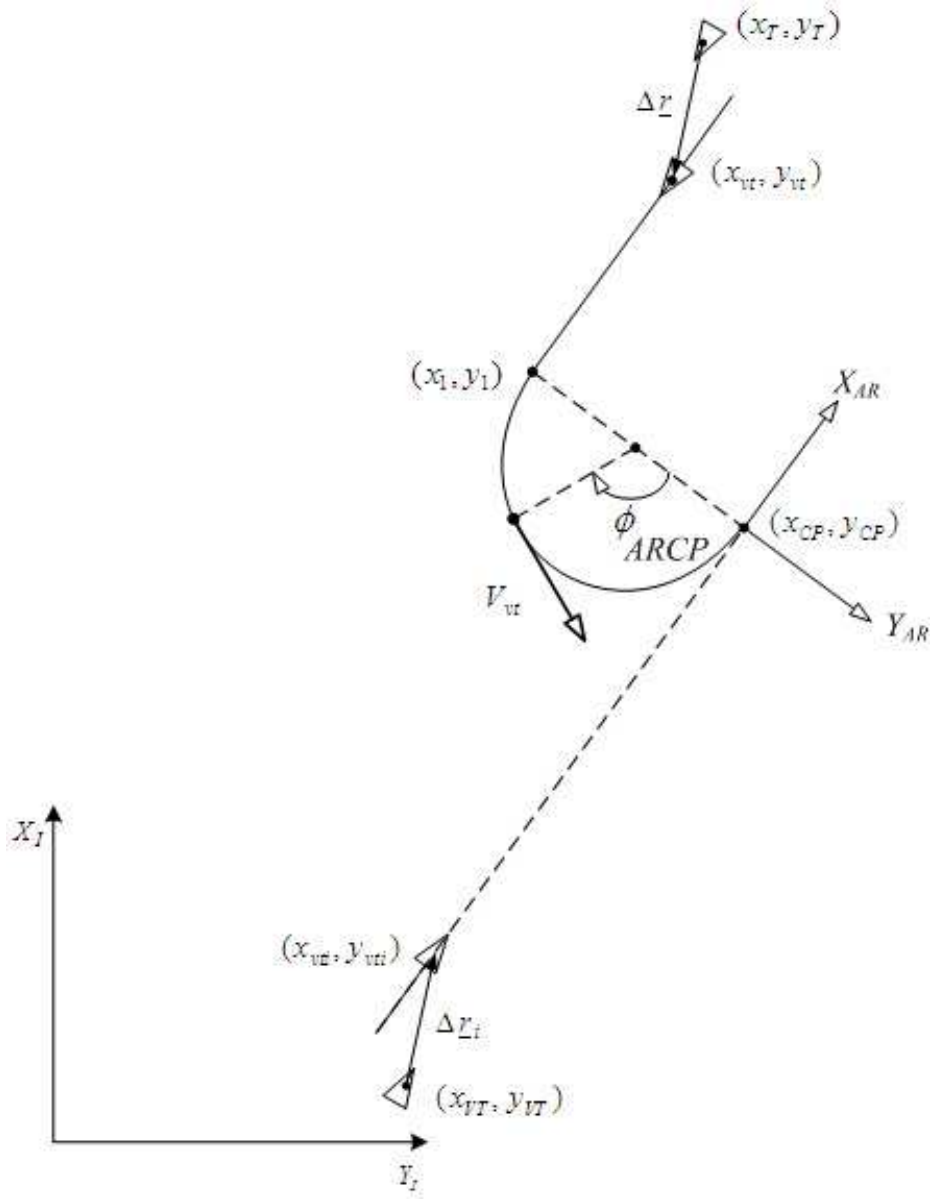

Figure 3.8. Diagram of rendezvous trajectory.

$$
\underline{\mathrm{r}}_{V T}=\underline{\mathrm{r}}_{v t i}-\triangle \underline{\mathrm{r}}
$$

Expressing the position vectors in terms of $x$ and $y$ coordinates in the inertial frame, Eq. (3.34) implies

$$
\begin{aligned}
& \triangle x=\left(x_{v t}-x_{T}\right) \\
& \triangle y=\left(y_{v t}-y_{T}\right)
\end{aligned}
$$

Substituting these equations in Eq. (3.36) results in 


$$
\begin{aligned}
& x_{V T}=x_{v t i}-\left(x_{v t}-x_{T}\right) \cos \phi-\left(y_{v t}-y_{T}\right) \sin \phi \\
& y_{V T}=y_{v t i}+\left(x_{v t}-x_{T}\right) \sin \phi-\left(y_{v t}-y_{T}\right) \cos \phi
\end{aligned}
$$

where $\left(x_{v t i}, y_{v t i}\right)$ are calculated in Eqs. (3.29) and (3.30). During the time when the virtual target is on the first straight leg or on the turn arc, the final form of Eqs. (3.39) and (3.40), becomes

$$
\begin{gathered}
x_{V T}=x_{C P}-\left(l_{s_{T 1}}+L\right) \cos \gamma-\left(x_{v t}-x_{T}\right) \\
y_{V T}=y_{C P}-\left(l_{s_{T 1}}+L\right) \sin \gamma-\left(y_{v t}-y_{T}\right)
\end{gathered}
$$

when the virtual target is on the aerial refueling line, after the turn is completed, using Eqs. (3.31) and Eqs. (3.32), Eqs. (3.39) and (3.40) become

$$
\begin{aligned}
& x_{V T}=x_{T} \\
& y_{V T}=y_{T}
\end{aligned}
$$

For the implementation of the virtual tanker concept for the receiver to follow, the velocity of the virtual tanker should also be known. This is calculated by taking the derivative of the $x_{V T}$ and $y_{V T}$ in Eqs. (3.41) and (3.42), which yield

$$
\begin{gathered}
\dot{x}_{V T}=-\left(\dot{l}_{s_{T}}+\dot{L}\right) \cos \gamma-\left(\dot{x}_{v t}-\dot{x}_{T}\right) \\
\dot{y}_{V T}=-\left(\dot{l}_{s_{T}}+\dot{L}\right) \sin \gamma-\left(\dot{y}_{v t}-\dot{y}_{T}\right)
\end{gathered}
$$

which are for when the virtual target is on the first straight leg or turning. After the turn, when the virtual target is on the aerial refueling line, Eqs. (3.43) and (3.44) imply

$$
\begin{aligned}
& \dot{x}_{V T}=\dot{x}_{T} \\
& \dot{y}_{V T}=\dot{y}_{T}
\end{aligned}
$$


CHAPTER 4

\section{SIMULATION AND ANALYSIS OF THE RESULTS}

\subsection{Introduction}

This chapter explains how the simulations are carried out and presents the simulation results and their analysis. The rest section focuses on the simulation of the tanker following the virtual target. It also demonstrates how the parameters of the nonlinear guidance logic are tuned for the tanker to fly along the aerial refueling orbit by the virtual target in the presence of the wind. The last section presents the simulation of the receiver following the virtual tanker along aerial refueling line to rendezvous with the actual tanker after ARCP. The section also gives the details of the motion of the virtual tanker synchronized with the actual tanker. It also shows how the receiver transition from following the virtual tanker to the actual tanker.

\subsection{Tanker aircraft}

This section describes the simulations environment and the results of the simulations for the tanker aircraft guided by the lateral acceleration command from the nonlinear guidance logic after converted to the yaw rate command to track the racetrack orbit pattern. The existing controller of the tanker aircraft would perfectly work to track the racetrack orbit with a commanded yaw rate schedule in the absence of wind. However, when prevailing wind is present, the trajectory generated by the commanded yaw rate schedule will be distorted relative to the inertial frame. Fig. 4.1 illustrates how the trajectory is distorted by prevailing wind when the tanker is flown with a yaw-rate command schedule that would generate a perfect racetrack without 
wind. The inner loop controller for the tanker aircraft consists of altitude hold, ve-

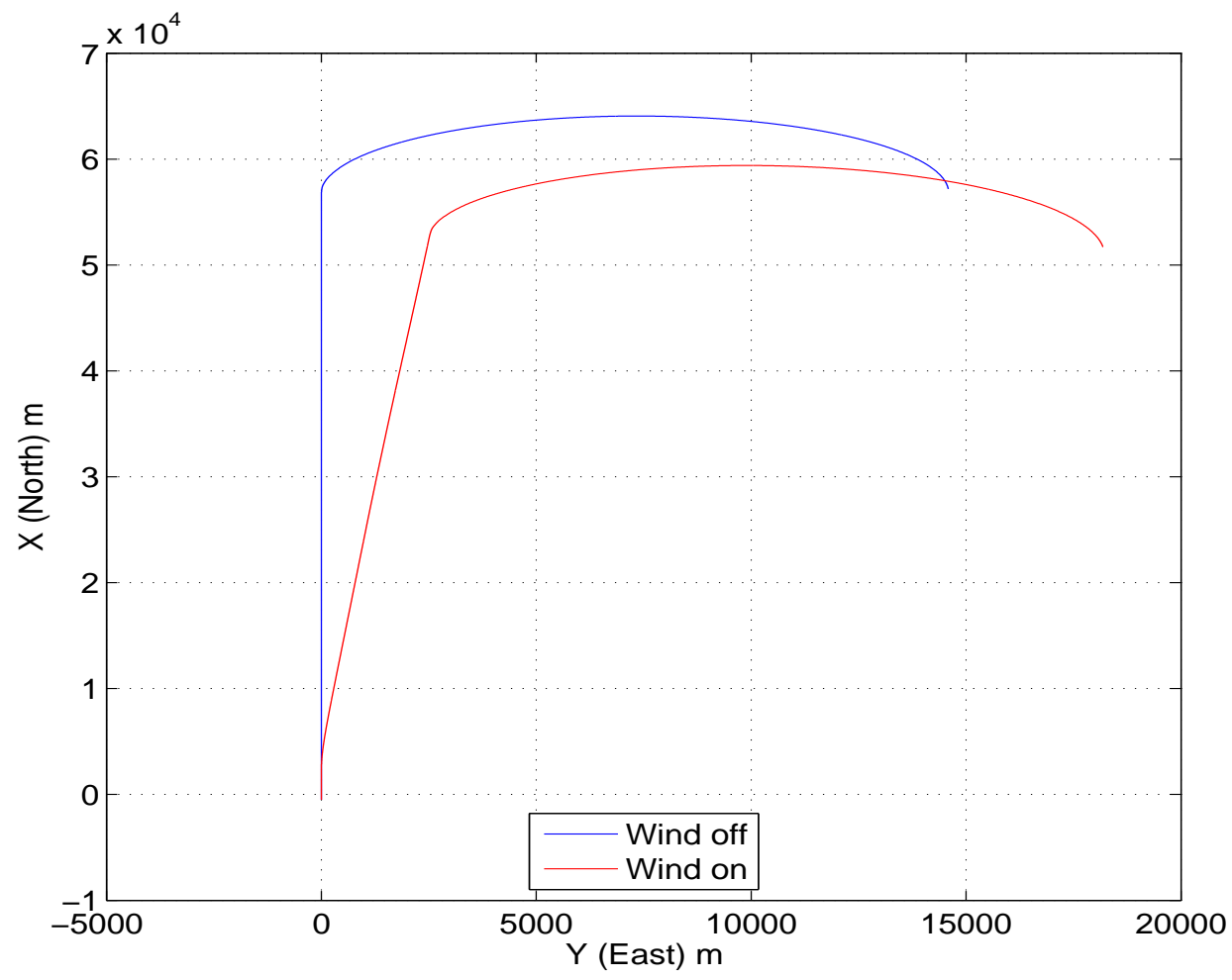

Figure 4.1. Tanker trajectory comparison with wind and no wind.

locity hold and yaw rate tracking, the controllers are developed in a prior work which are designed based on the four nominal conditions as explained in chapter 2 . The tanker aircraft is commanded to fly with constant velocity $V_{c}=190 \mathrm{~m} / \mathrm{s}$ at altitude of 7010 meters.

\subsubsection{No wind condition}

This section analyzed the performance of the guidance and the controller for tracking a straight line trajectory and circular trajectory in the absence of wind there are two sets of cases simulated. The first set analyzes the effect of initial lateral 
distance from the straight line trajectory. This is to see how far away the guidance and control can move the tanker to the desired trajectory. The second set of simulations investigates the effect of the parameter of the nonlinear guidance logic on the tracking performance. In both sets of simulations, the virtual target flies with $190 \mathrm{~m} / \mathrm{s}$ speed on the aerial refueling orbit and the tanker is commanded to fly with the same speed.

In the first set of simulations, refer to as "CASE - I", the virtual target starts from the origin, $(0,0)$ in the inertial frame, moving North. Four simulation cases with the following initial positions of the tanker are on: $(-5000,0),(-5000,15)$, $(-5000,500)$ and $(-5000,1000)$. In all cases, the length parameter of the guidance logic is kept the same as $L_{1}=5000$.

In the second sets, referred to as "CASE - II", of the simulations, the virtual tanker does the same as in the previous simulation cases. The initial position of the tanker is $(-2000,500) \mathrm{m}$. Four cases are simulated with different nonlinear guidance parameters as $L_{1}=100, L_{1}=500, L_{1}=1000, L_{1}=2000$. 


\subsubsection{CASE-I results}

Fig. 4.2 shows the trajectories of the virtual target and the tanker in the $\mathrm{x}-\mathrm{y}$ plane. With all four initial conditions, the tanker aircraft is successfully guided to the desired trajectory as the virtual target moves on a straight line. In all four cases the tanker aircraft has some deviation from the desired path while the virtual target is turning. Fig. 4.3 shows the deflections of aileron, elevator and rudder. As the lateral offset increases, it requires larger control action to move the aircraft to the desired path. In all cases, the control surface deflections are within their respective limits. Fig. 4.4 shows the comparison of commanded yaw rate as generated by the nonlinear guidance logic versus the yaw rate response of the tanker. As larger yaw rate commands. In all cases, the tanker follows the yaw rate commands very well. Fig. 4.5 shows the comparison of commanded speed and the speed response of the tanker. The drops in the commanded speed during the turns are due to the proportional controller implemented. The gain $K_{p}$ of the controller in these simulation is 0.0001 .

As the lateral distance increasing the tanker aircraft use higher control effort to intercept the desired trajectory which are shown in the figures that the initial response are oscillated. Therefore the conclusion can be drawn that the initial responses of the tanker aircraft is deteriorated when the lateral distance increasing. 


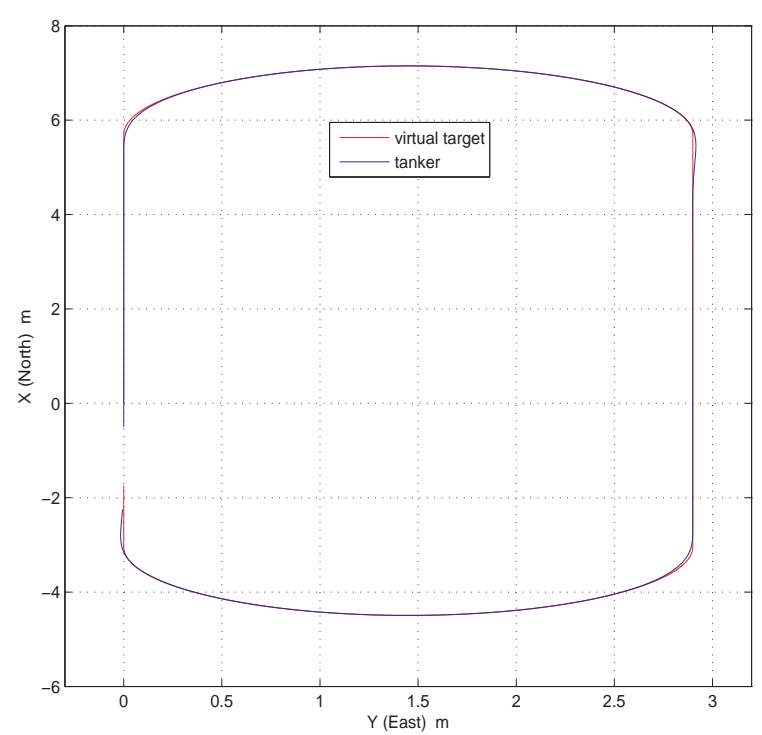

(a)

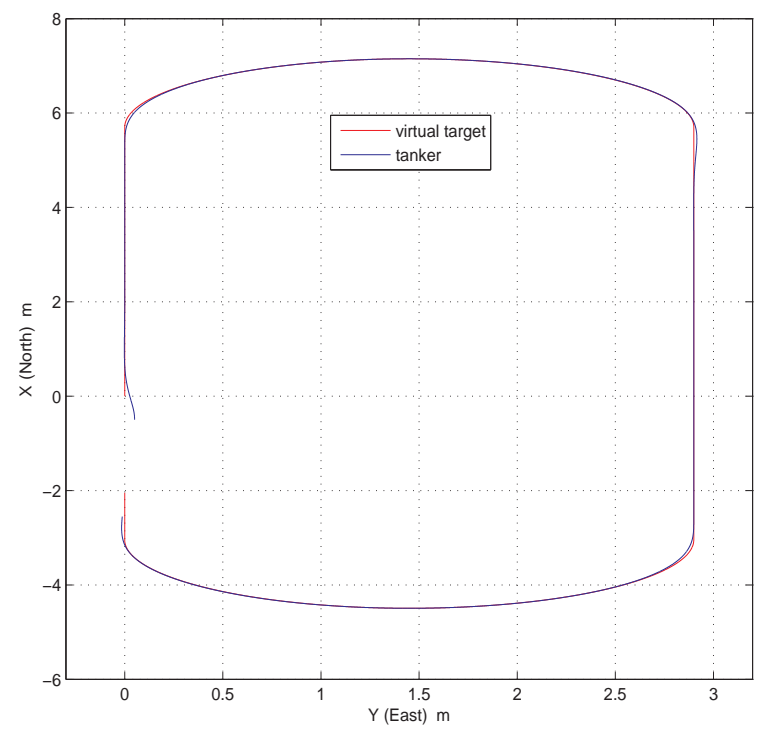

(c)

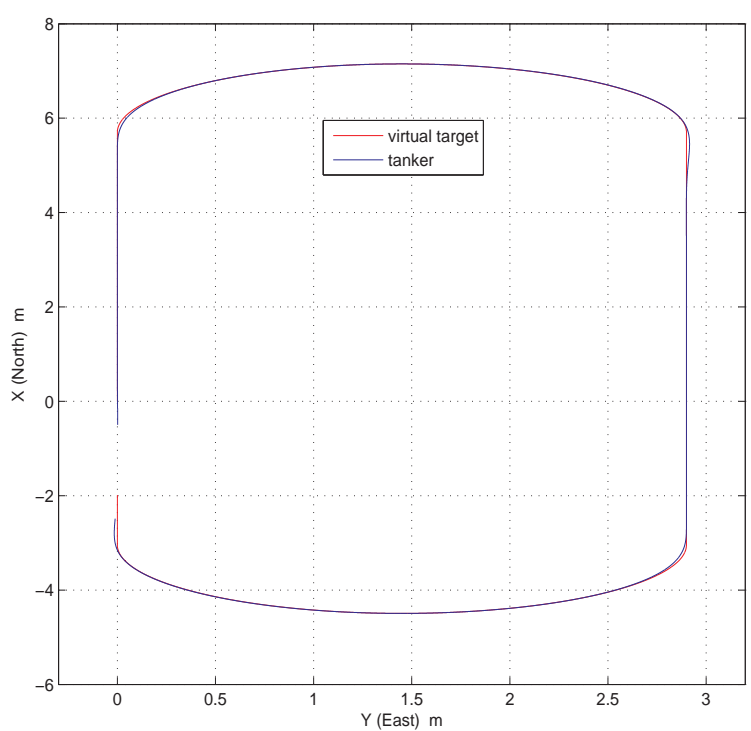

(b)

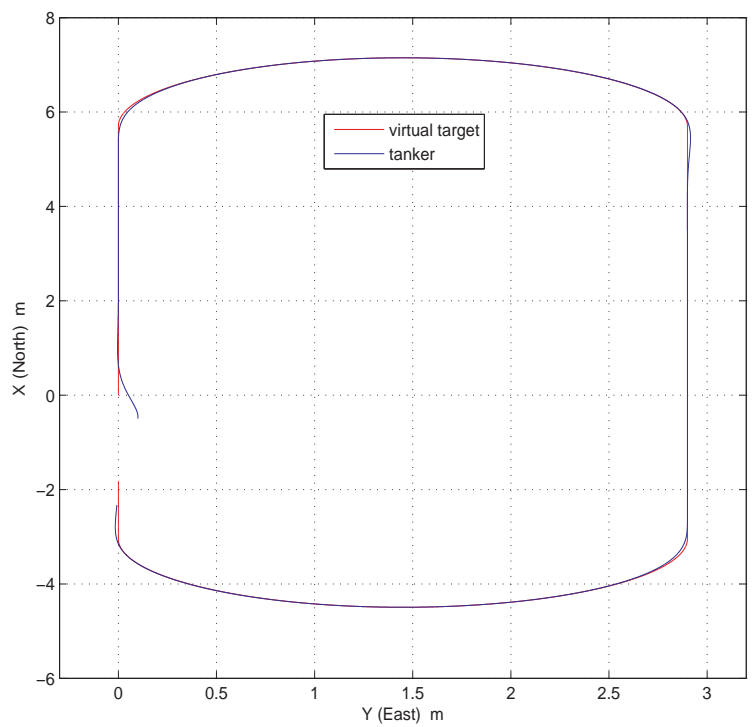

(d)

Figure 4.2. Trajectory comparison when tanker initially at (a) $(-5000,0)$ (b) $(-5000,100)(\mathrm{c})(-5000,500)$ and $(\mathrm{d})(-5000,1000)$. 


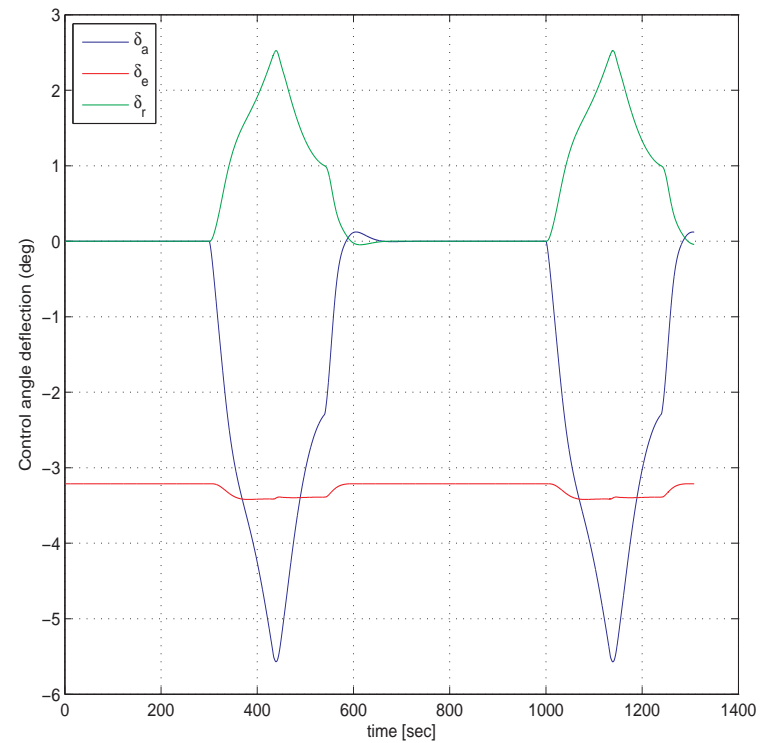

(a)

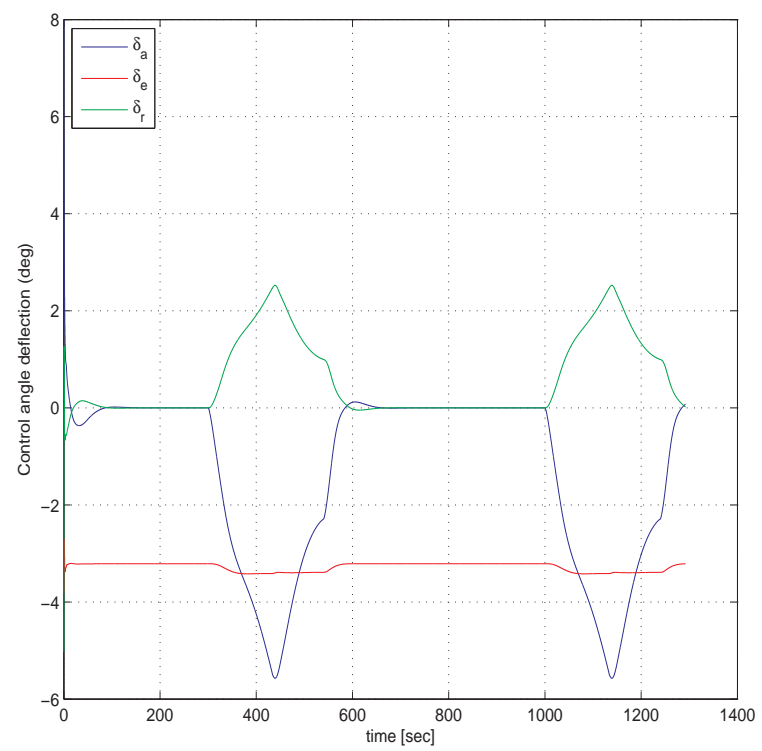

(c)

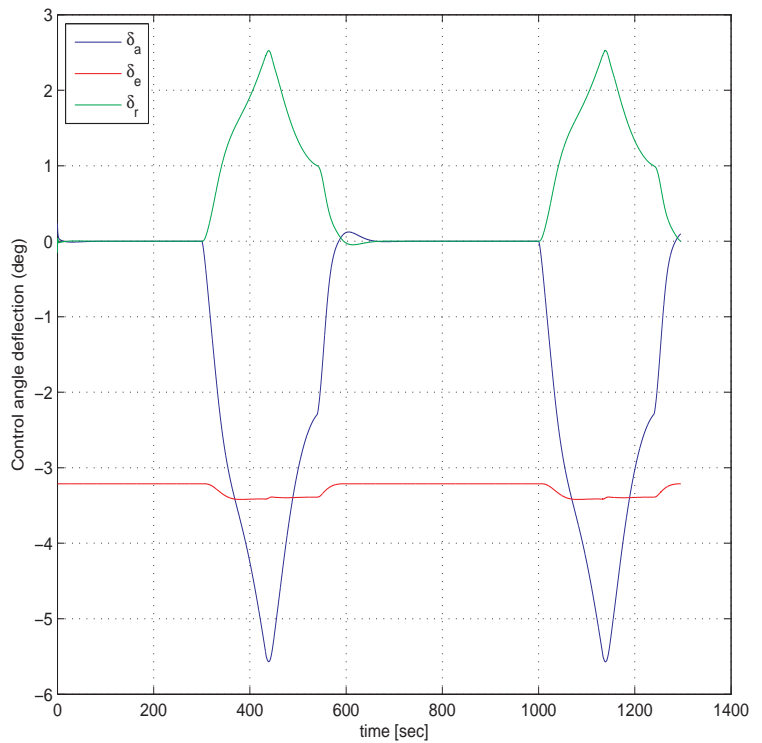

(b)

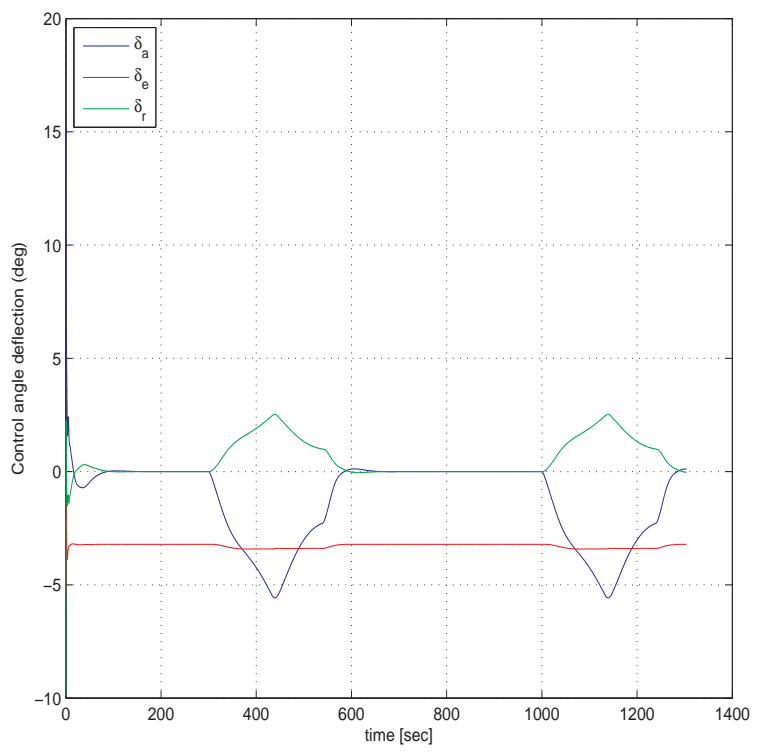

(d)

Figure 4.3. Control deflection when tanker initially start at (a) $(-5000,0)$ (b) $(-5000,100)(\mathrm{c})(-5000,500)$ and $(\mathrm{d})(-5000,1000)$. 


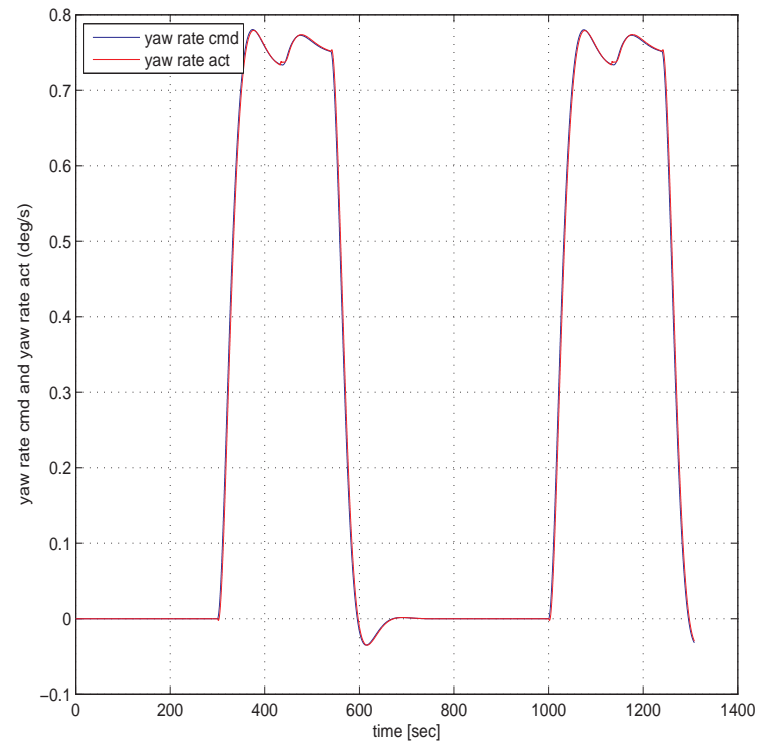

(a)

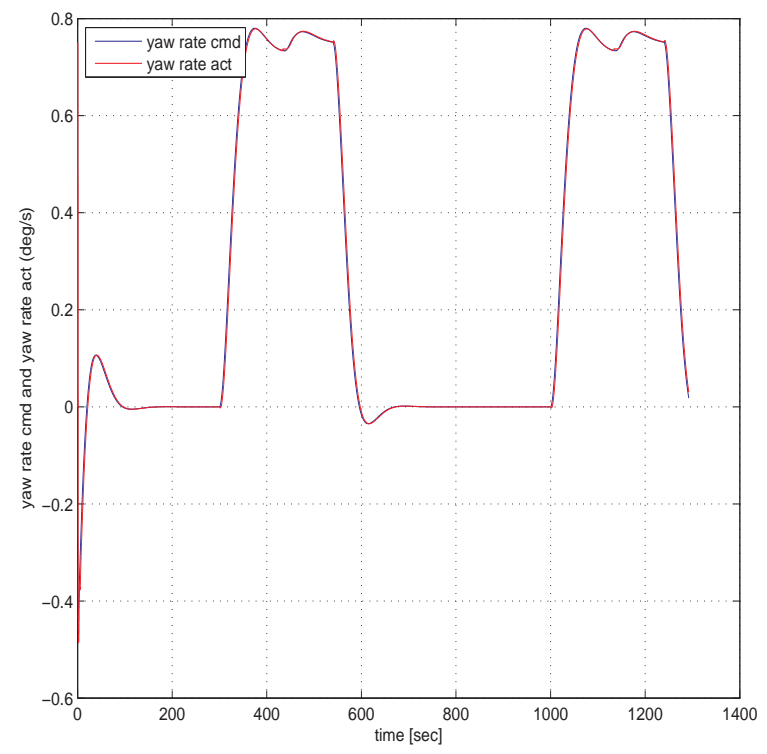

(c)

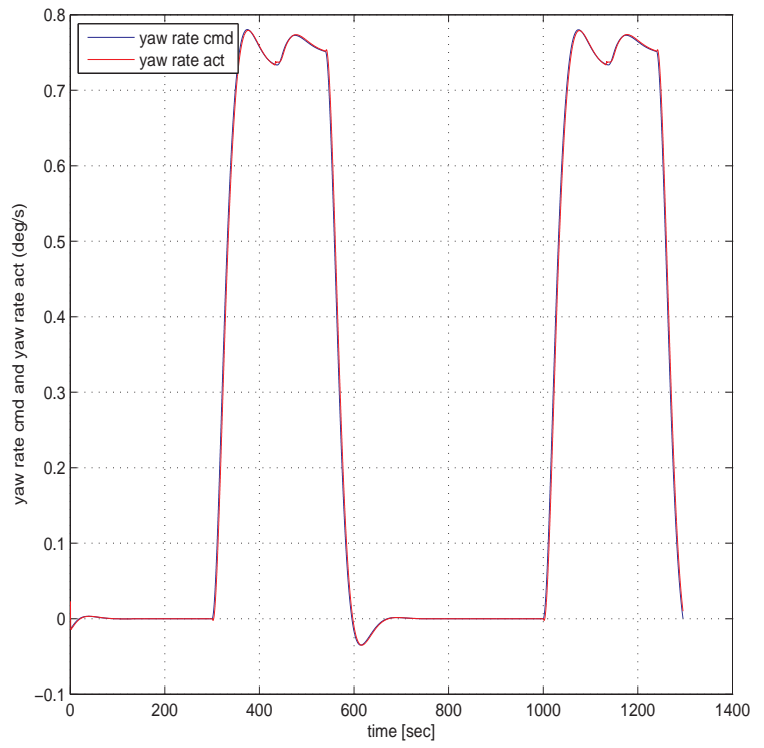

(b)

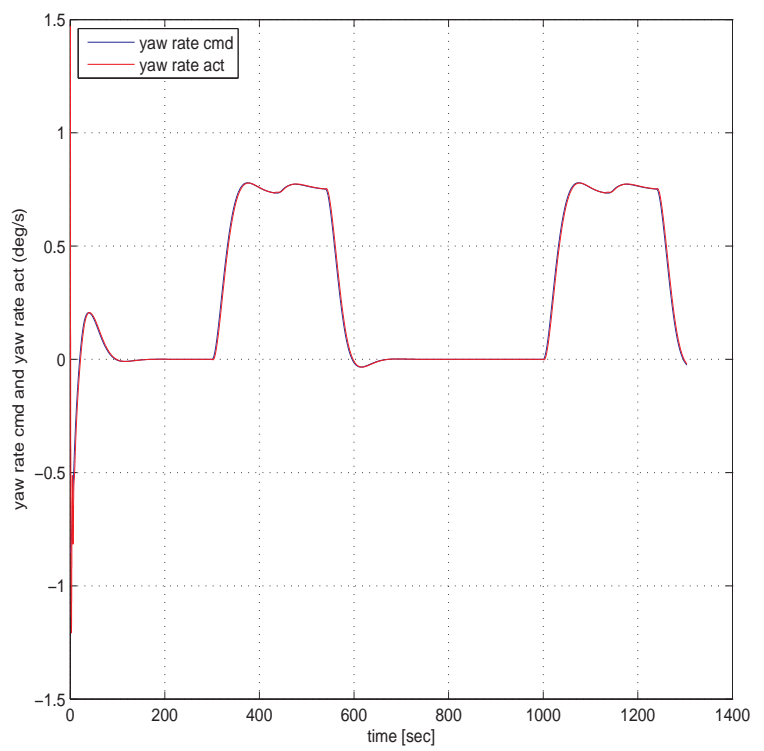

(d)

Figure 4.4. Comparison of yaw rate when tanker initially start at (a) $(-5000,0)(\mathrm{b})$ $(-5000,100)(\mathrm{c})(-5000,500)$ and $(\mathrm{d})(-5000,1000)$. 


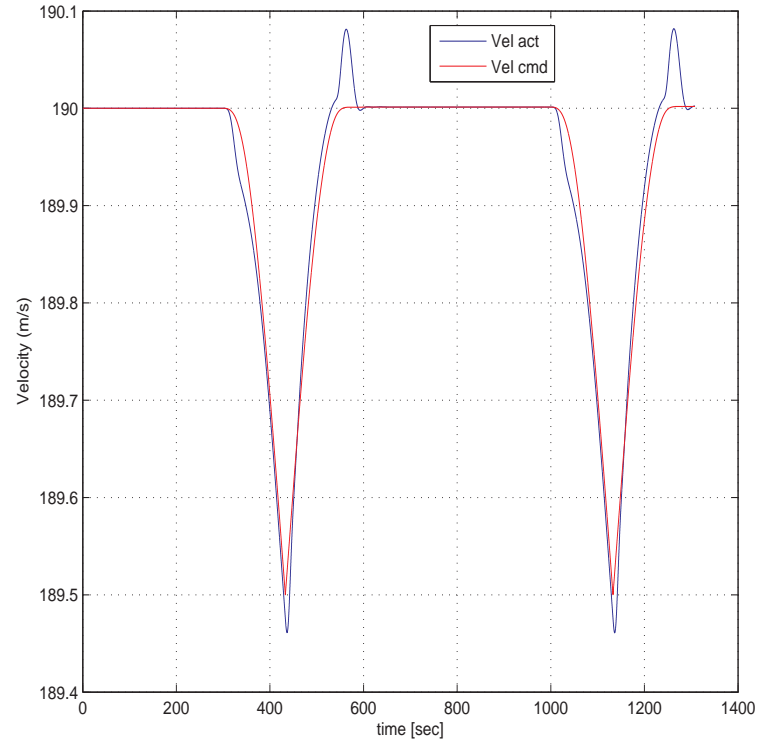

(a)

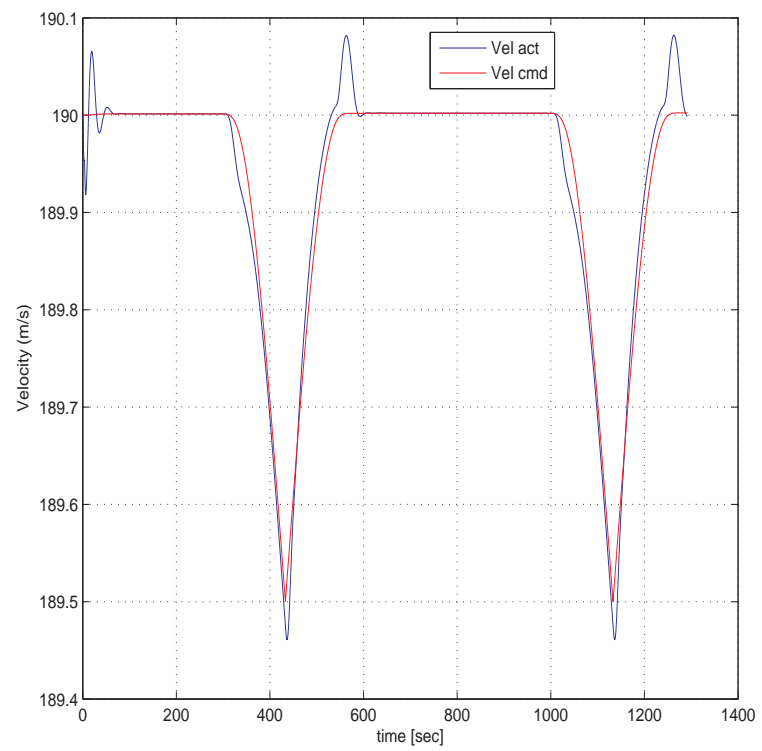

(c)

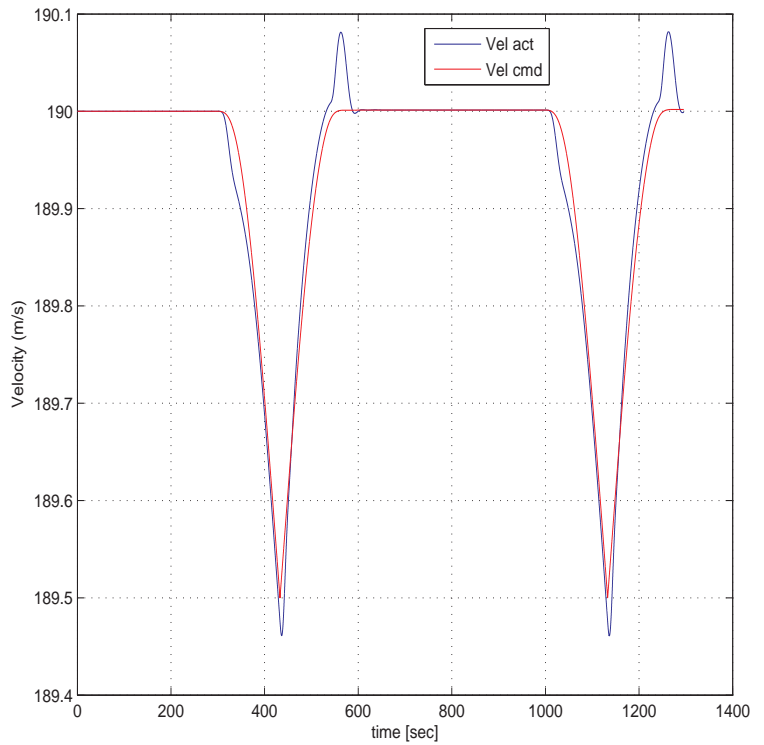

(b)

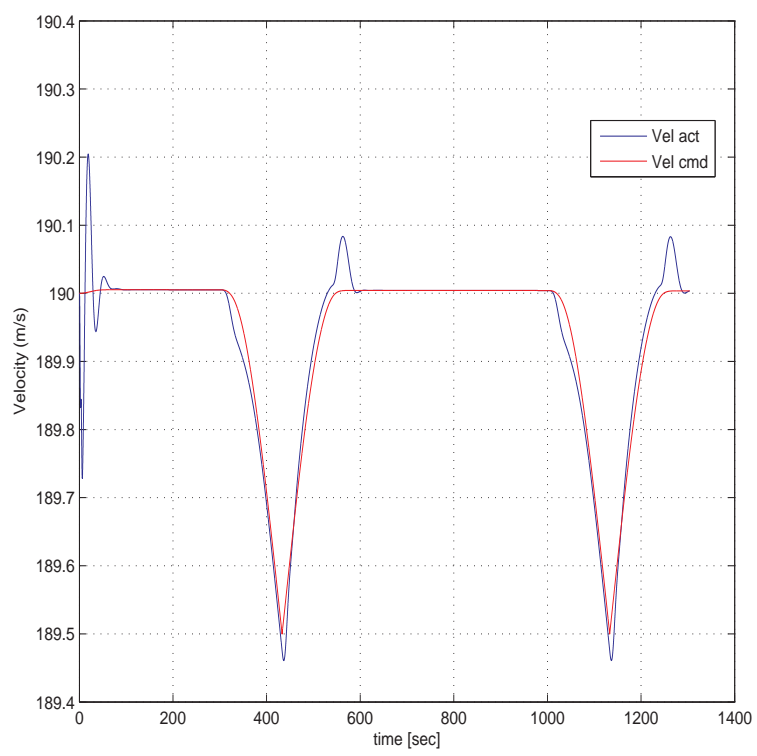

(d)

Figure 4.5. Comparison of commanded velocity when tanker initially start at (a) $(-5000,0)(\mathrm{b})(-5000,100)(\mathrm{c})(-5000,500)$ and $(\mathrm{d})(-5000,1000)$. 


\subsubsection{CASE-II results}

Simulation results with four different $L_{1}$ values are presented. At the initial time, the tanker is $2000 \mathrm{~m}$ behind and $500 \mathrm{~m}$ to the right of the virtual target the values of $L_{1}$ are $L_{1}=100, L_{1}=500, L_{1}=1000, L_{1}=2000$. Fig. 4.6 shows the trajectory comparison between the tanker aircraft and the virtual target. The results show that the tanker aircraft is capable of tracking the desired trajectory. Fig. 4.7 shows the control signals of the tanker aircraft which are the aileron, elevator and rudder deflections. Figure 4.8 shows the comparison between commanded yaw rate and actual yaw rate of the tanker aircraft. Figure 4.9 shows the comparison between commanded velocity and the actual velocity of the tanker aircraft.

The simulation results show that the smaller $L_{1}$ values produce more control effects and may lead to instability. 


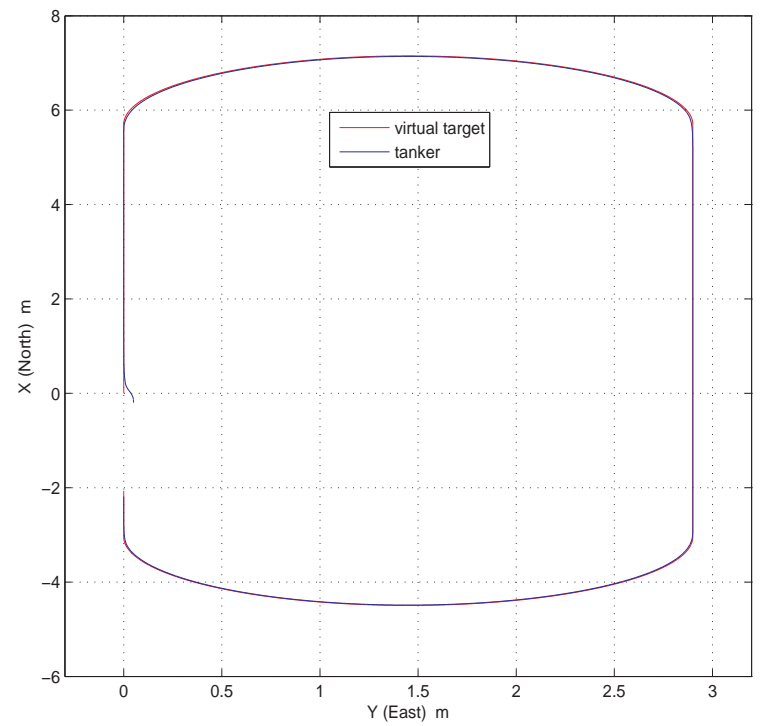

(a)

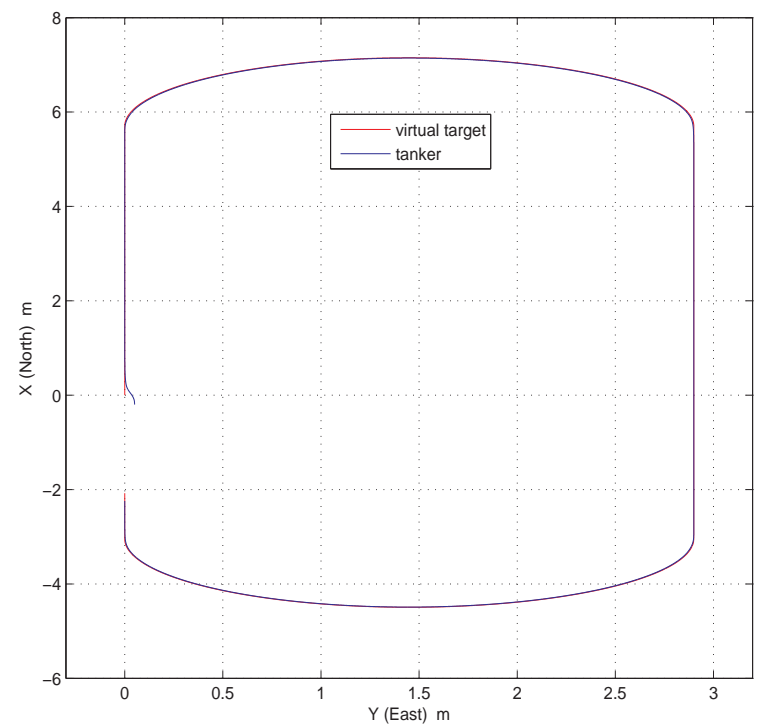

(c)

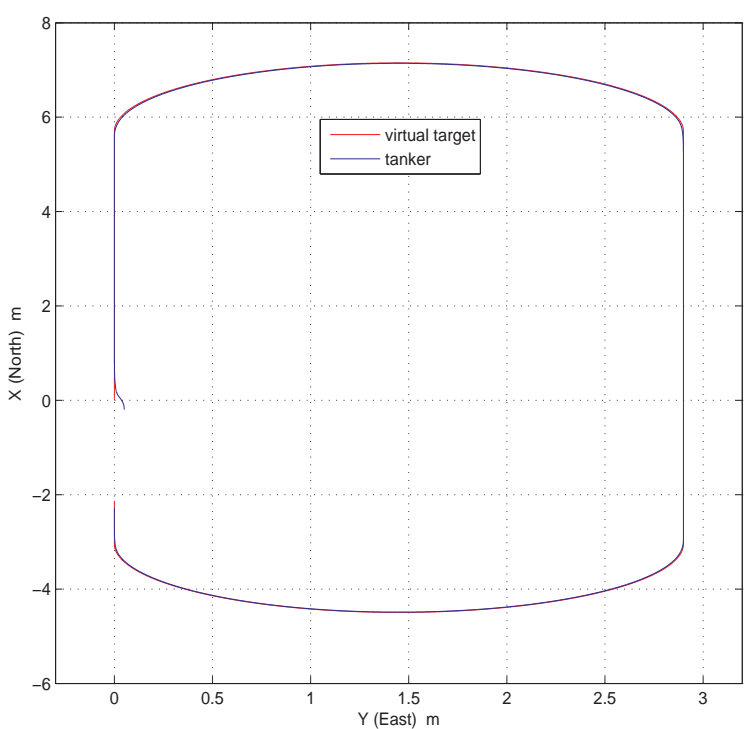

(b)

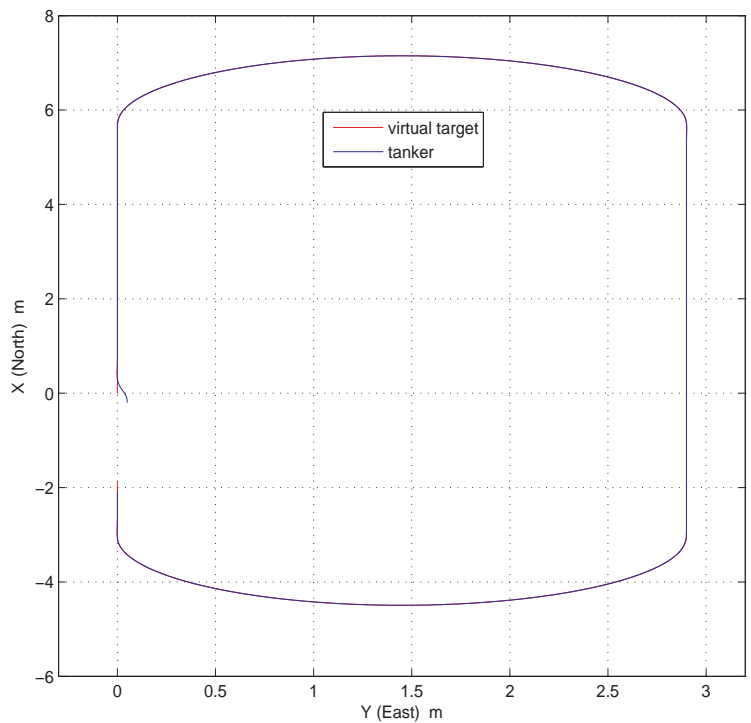

(d)

Figure 4.6. Trajectory comparison when (a) $L 1=100$ (b) $L 1=500$ (c) $L 1=1000$ and (d) $L 1=2000$. 


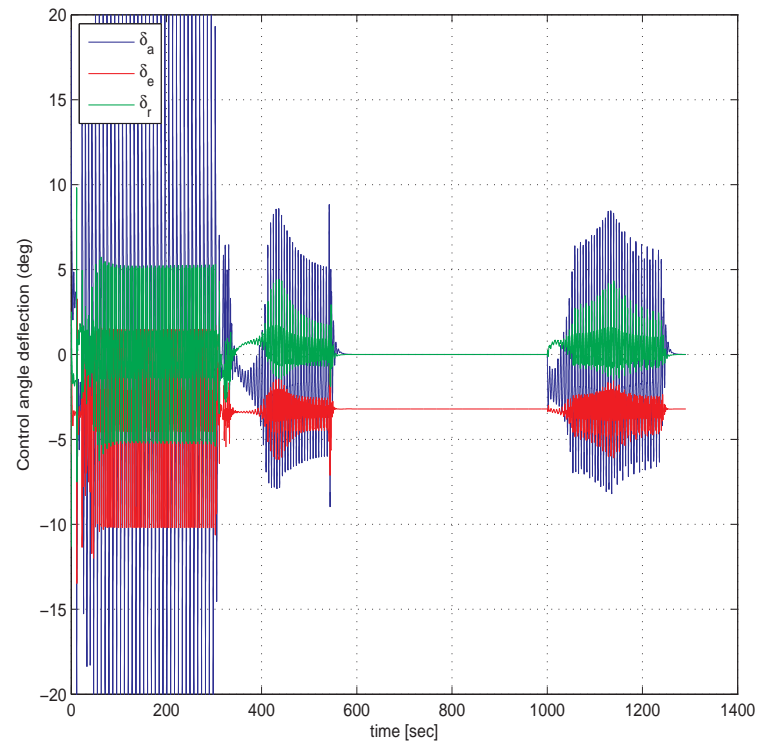

(a)

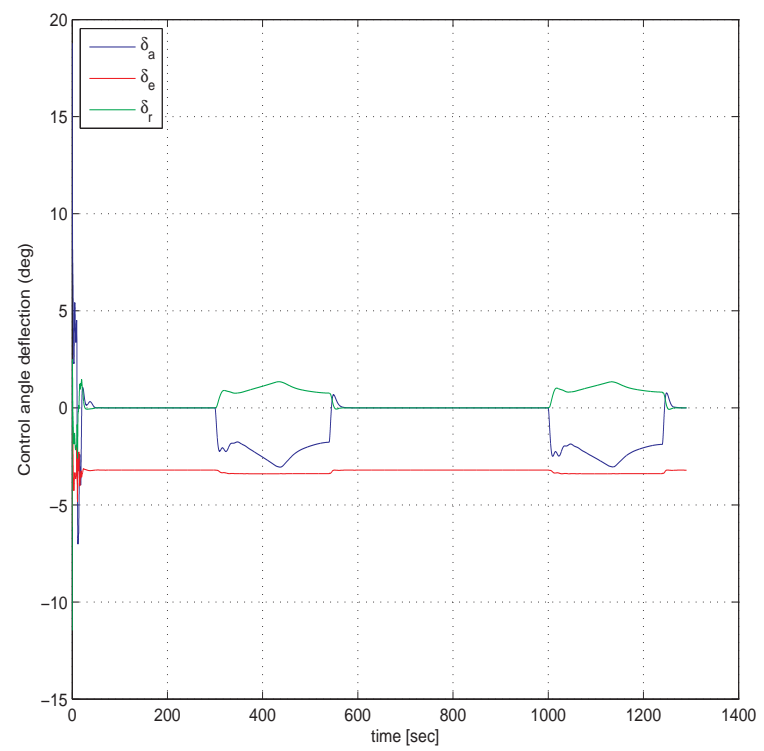

(c)

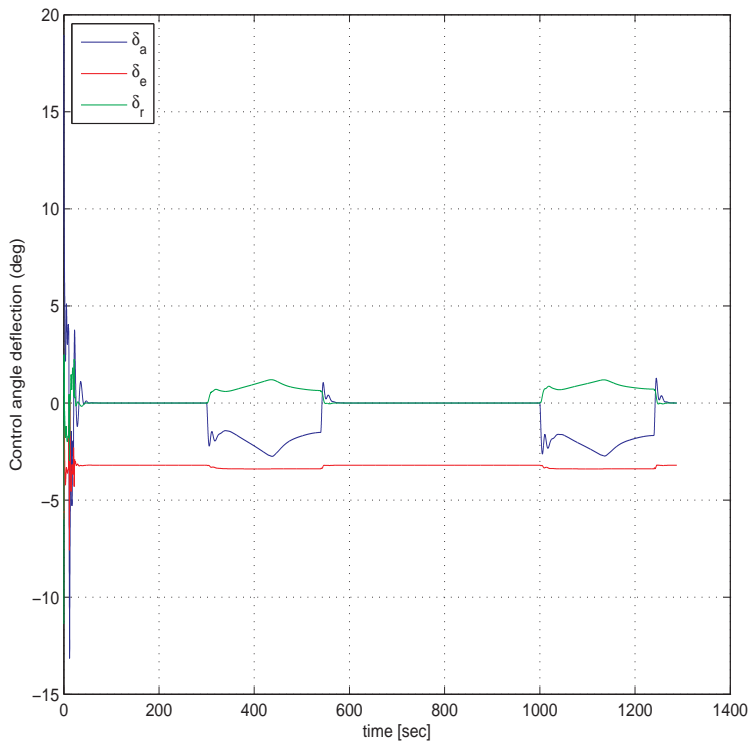

(b)

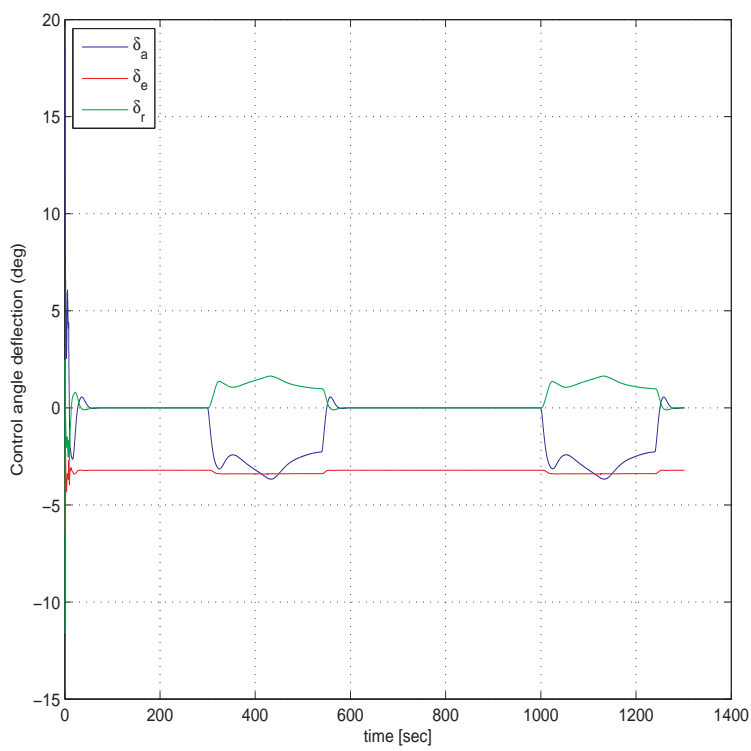

(d)

Figure 4.7. Control deflection when (a) $L 1=100$ (b) $L 1=500$ (c) $L 1=1000$ and (d) $L 1=2000$. 


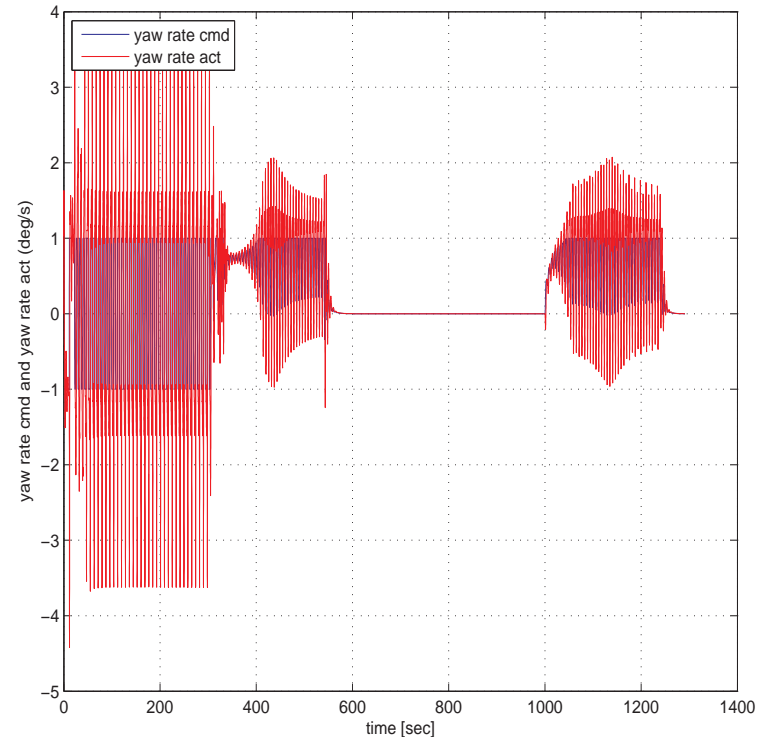

(a)

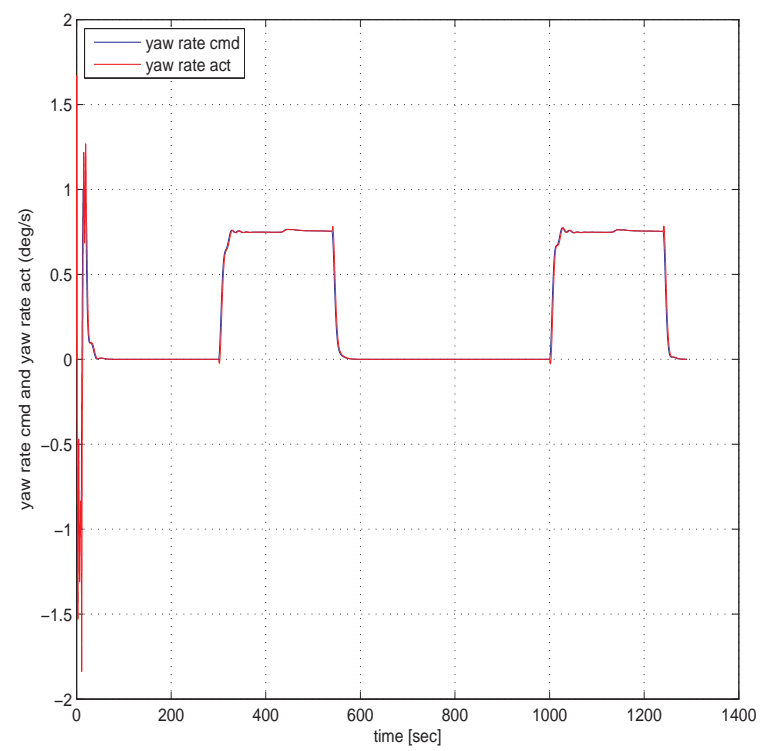

(c)

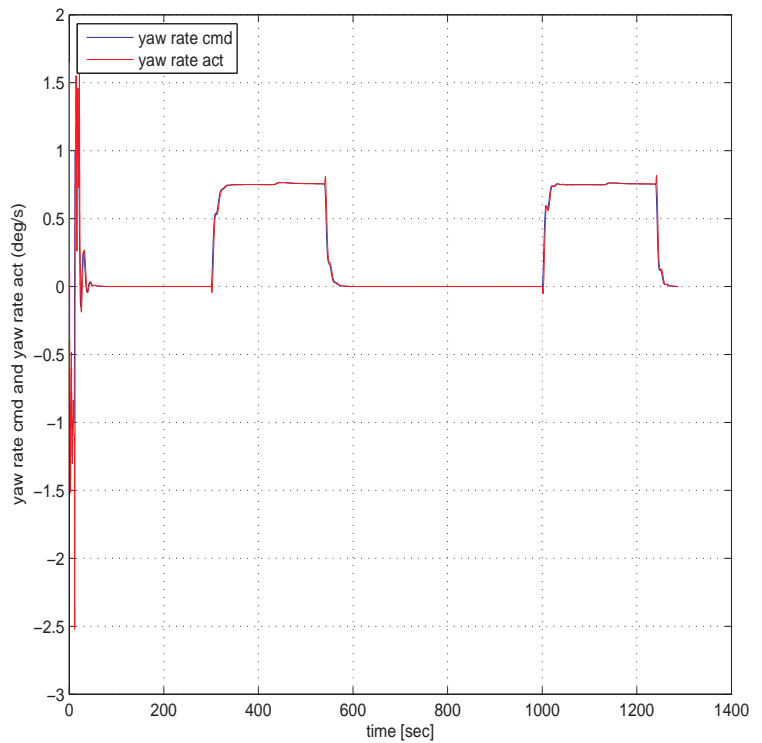

(b)

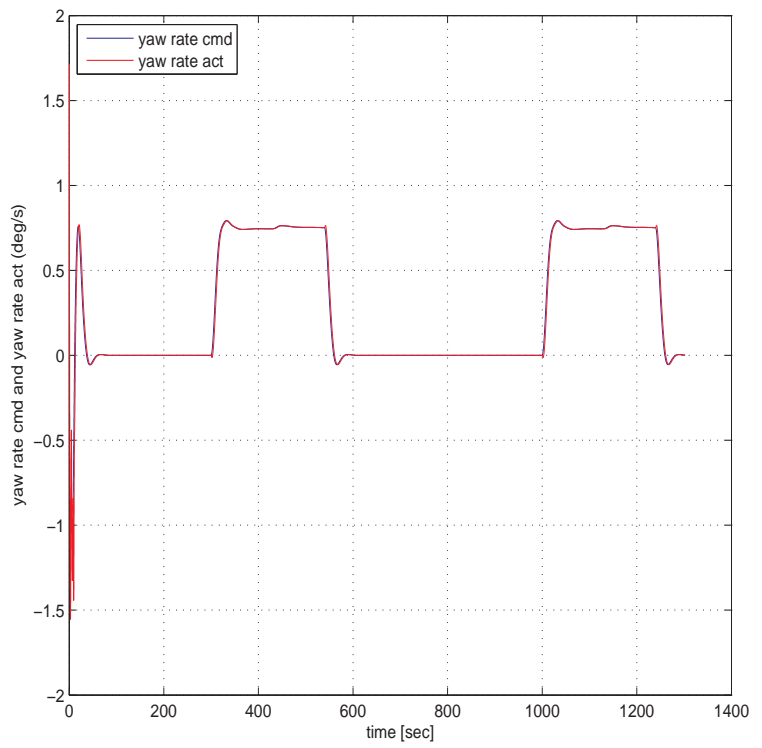

(d)

Figure 4.8. Comparison of yaw rate when (a) $L 1=100$ (b) $L 1=500$ (c) $L 1=1000$ and $(\mathrm{d}) L 1=2000$. 


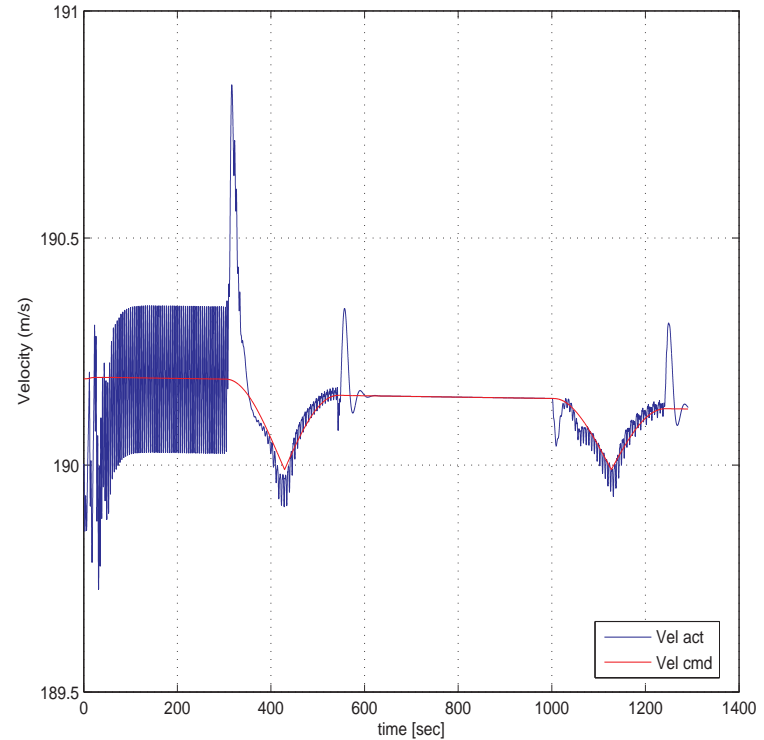

(a)

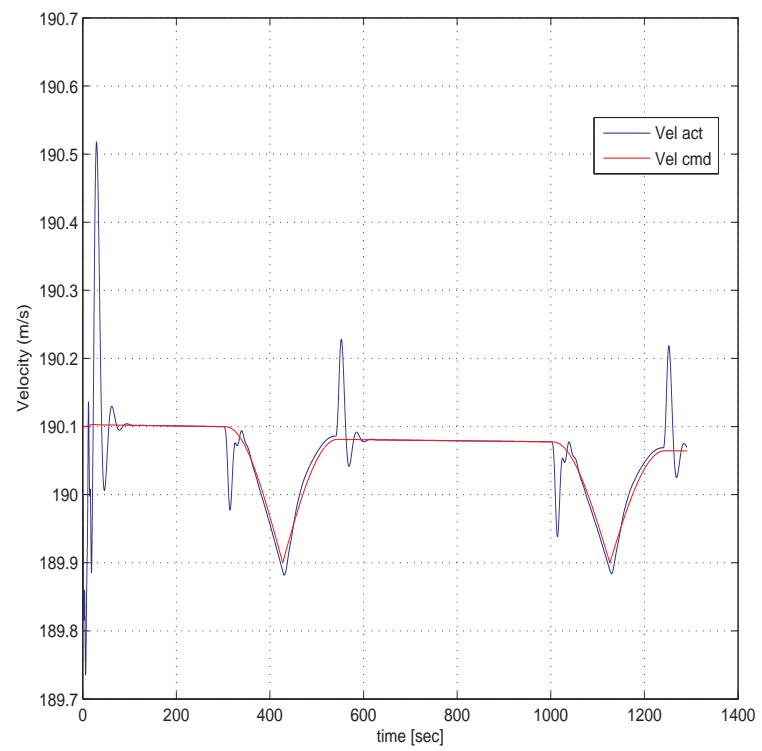

(c)

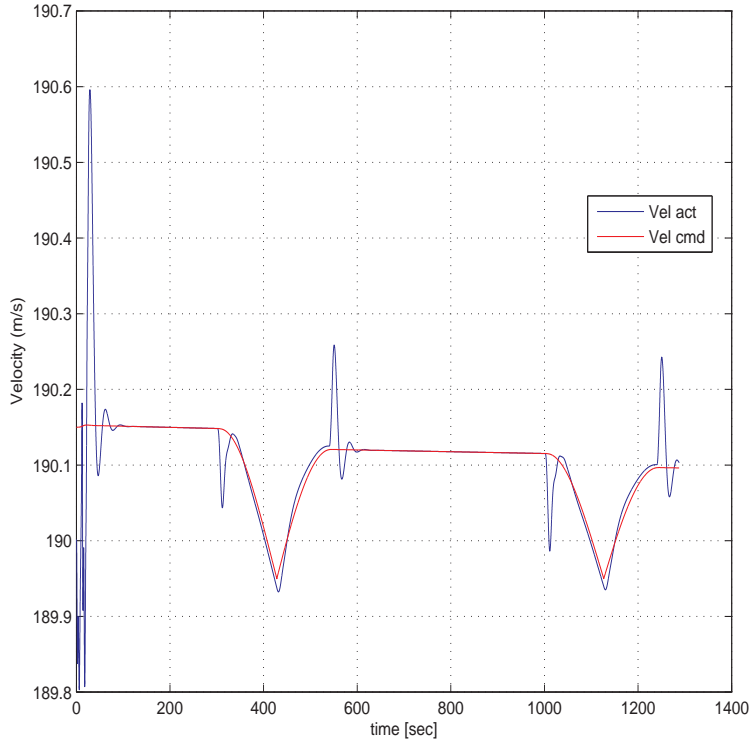

(b)

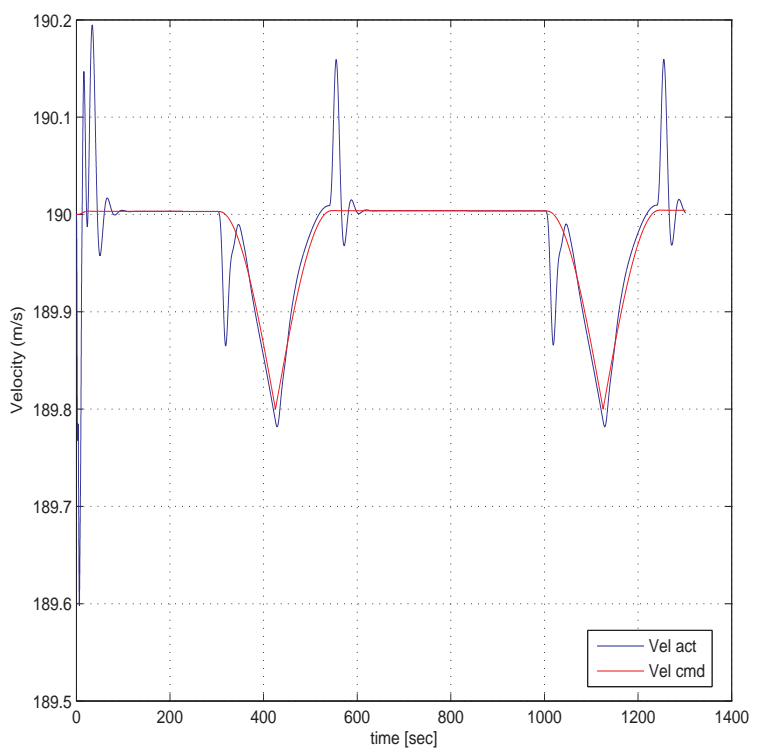

(d)

Figure 4.9. Comparison of commanded velocity when (a) $L 1=100$ (b) $L 1=500$ (c) $L 1=1000$ and (d) $L 1=2000$. 


\subsubsection{Wind condition}

In this section, the performance of the tanker flying on the refueling orbit is evaluated under the effect of a realistic prevailing wind exposure. Note in Fig. 4.10 that the prevailing wind has varying magnitude and direction during the rendezvous operation. Overall, the wind blows in the southeast direction. Note that the simulation starts with zero wind and then the wind is gradually turned on up to its normal level. The simulation is set up similar to the no-wind case discussed above. However, the simulation results show that due to the prevailing wind, when acting as tailwind, causes the tanker aircraft to catch up with and pass the virtual target. This is because the virtual target is not affected by the prevailing wind as its trajectory is formulated relative to the inertial frame. When the tanker ends up flying ahead of the virtual target, which it is supposed to follow, the nonlinear guidance algorithm no longer generates feasible yaw rate command. To overcome this problem the proportional controller is added to the velocity command of the tanker aircraft as explained in Chapter 3 .

In this section, the effect of varying the gain of the proportional control is illustrated. In these simulation, the virtual target trajectory is the same as before while the tanker starts $5000 \mathrm{~m}$ behind and $5 \mathrm{~m}$ to the right of the virtual target the parameter of the nonlinear guidance is set as $L_{1}=5000$. Fig. 4.11 shows the trajectory comparison between tanker and virtual target with various values of $K_{p}$ as $K_{p}=1$, $K_{p}=0.1, K_{p}=0.01, K_{p}=0.001$, in no case does the tanker follow the virtual target. Fig. 4.12 shows the simulation results when the gain $K_{p}$ is 0.0001 . With this value of $K_{p}$, The tanker aircraft capable of following the virtual target. Fig. 4.12 shows the trajectory comparison between tanker and virtual target with the prevailing wind. The tanker aircraft is capable of following the desired trajectory even in the presence of the prevailing wind. Fig. 4.13 shows the comparison of the position between the 


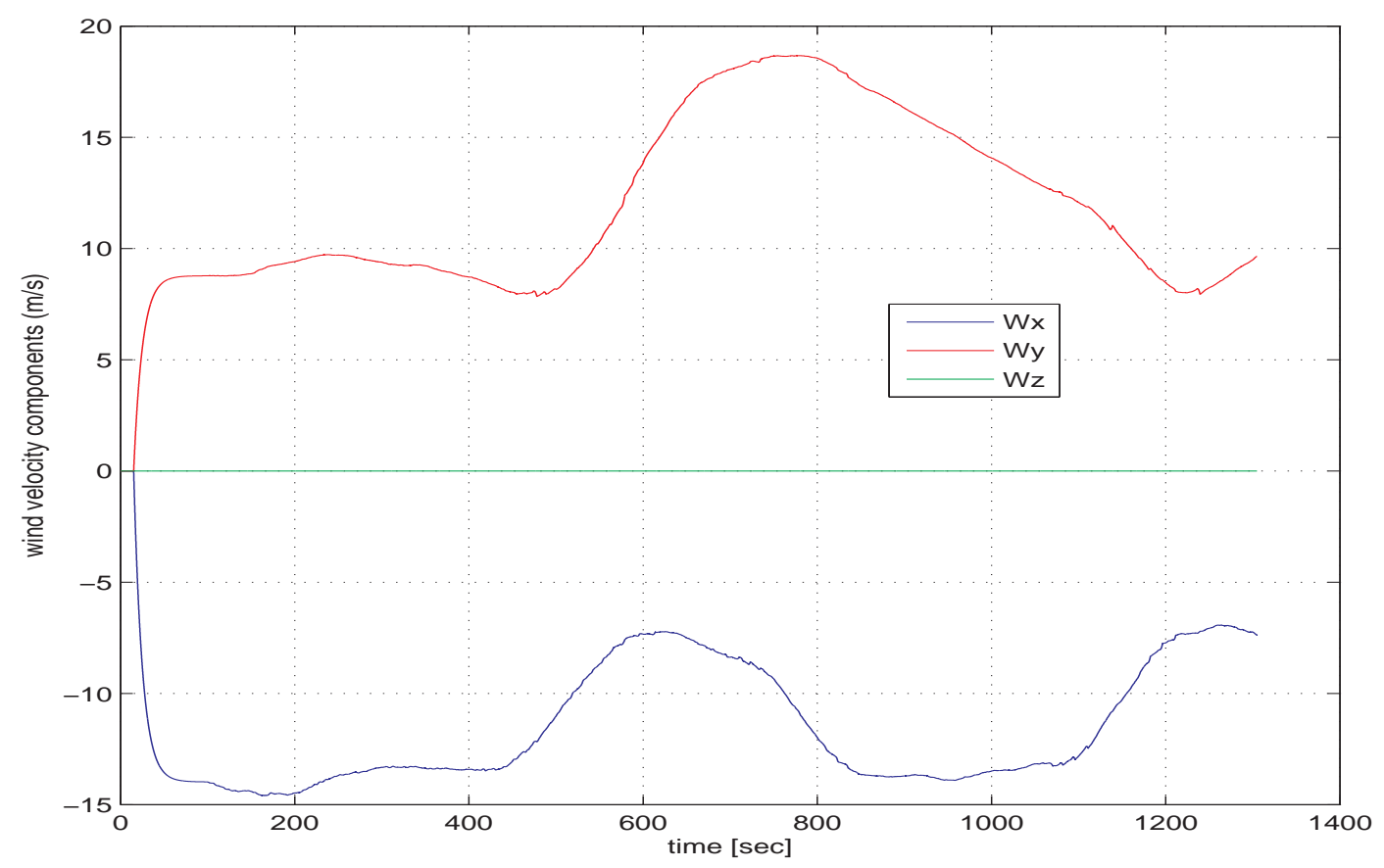

Figure 4.10. Wind component versus time.

tanker and the virtual target. Fig. 4.14 shows the relative position between the tanker and the virtual target. Fig. 4.15 shows the control surface deflections of the taker aircraft. Fig. 4.16 shows the comparison of the commanded velocity and the actual tanker's velocity. Figs. 4.17 and 4.18 show the comparison of the commanded yaw rate and the actual yaw rate of the tanker and the time history of yaw angle, respectively.

The simulations results shown that this guidance logic and the controller are capable of guiding the tanker aircraft to track the desired trajectory with fairly good performance in the presence of the prevailing wind, even during the turns. 


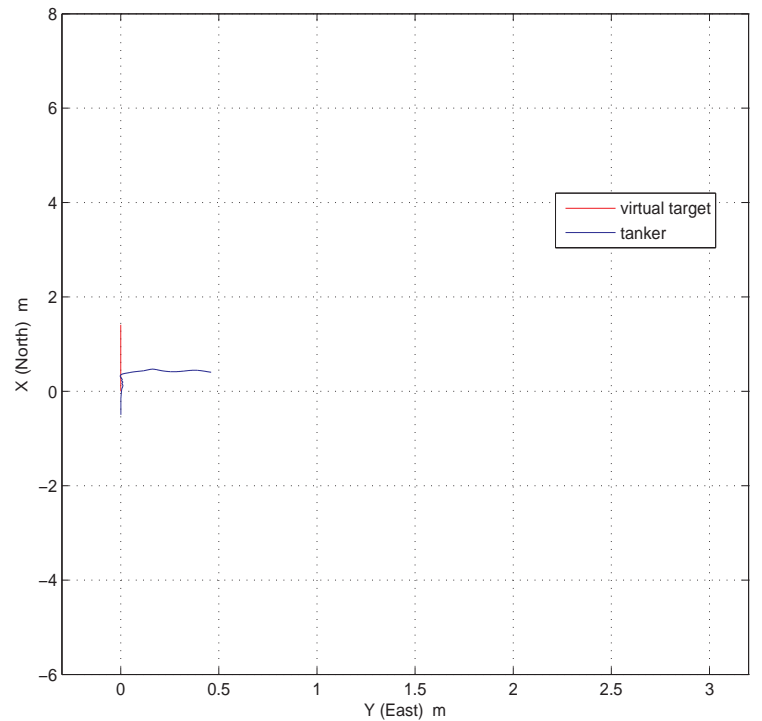

(a)

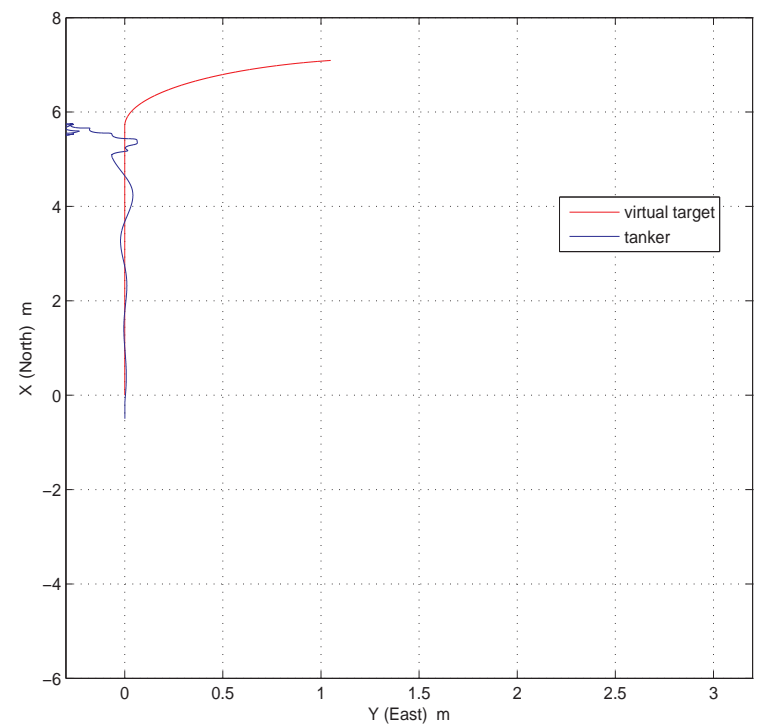

(c)

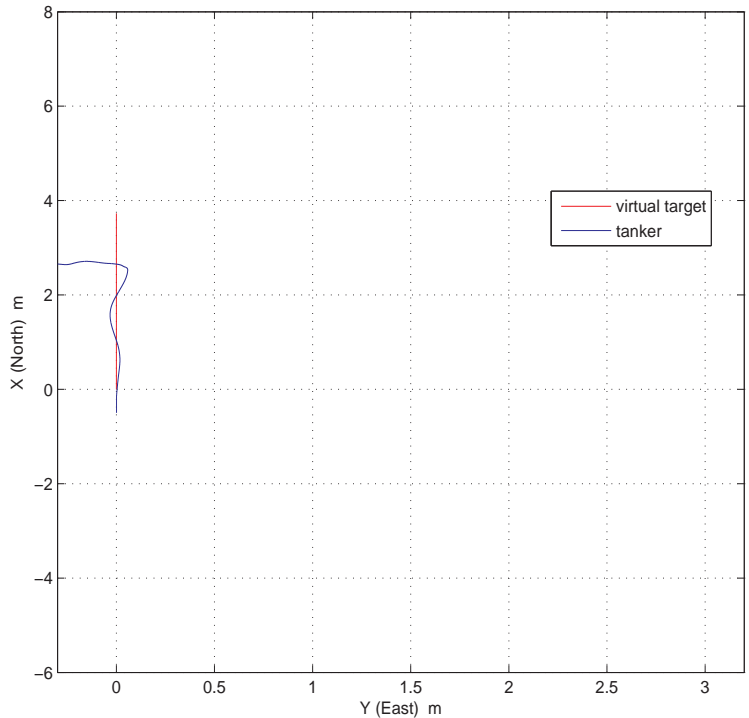

(b)

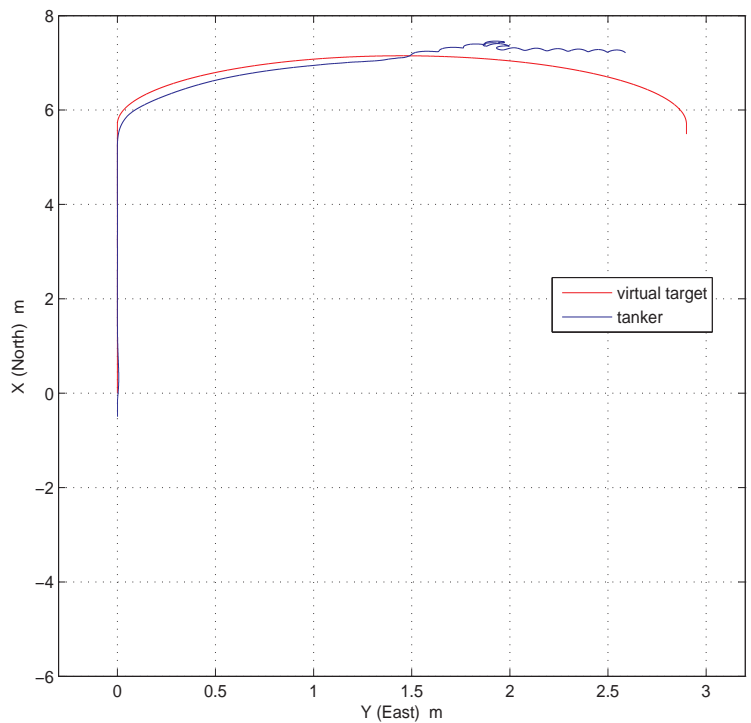

(d)

Figure 4.11. Trajectory comparison when (a) $K p=1$ (b) $K p=0.1$ (c) $K p=0.01$ and (d) $K p=0.001$. 


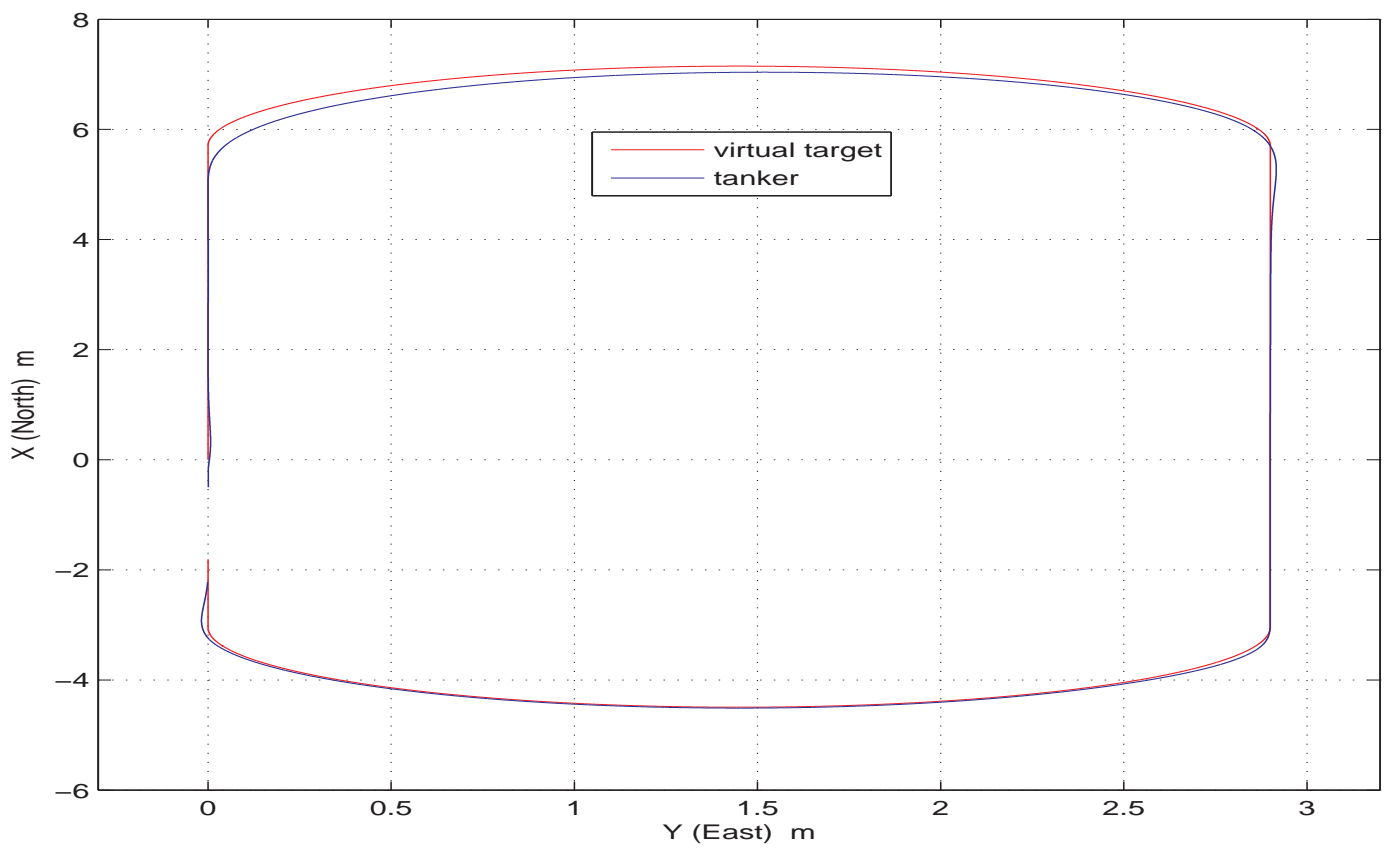

Figure 4.12. Trajectory comparison between tanker and virtual target.
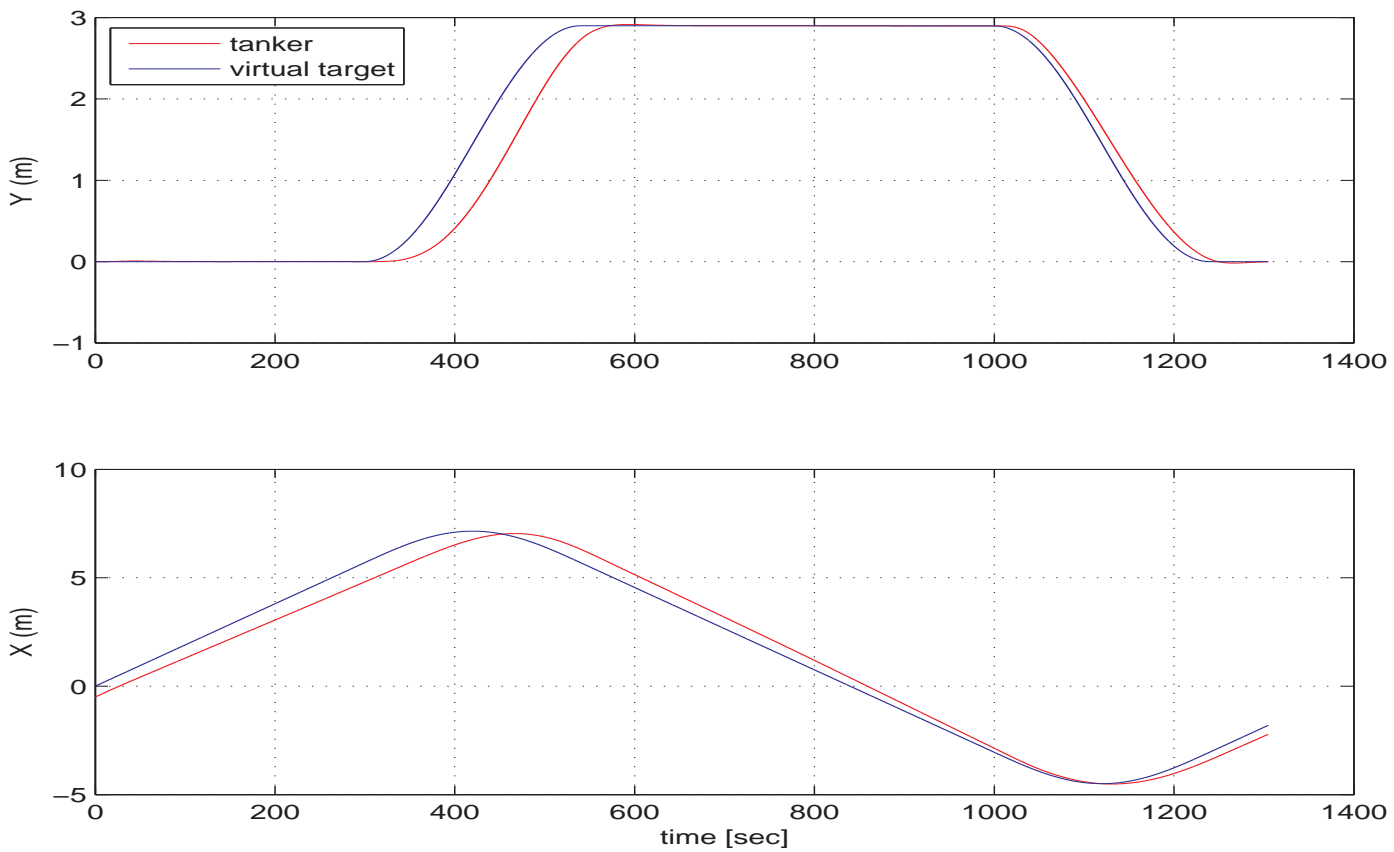

Figure 4.13. Comparison of position between tanker and virtual target. 

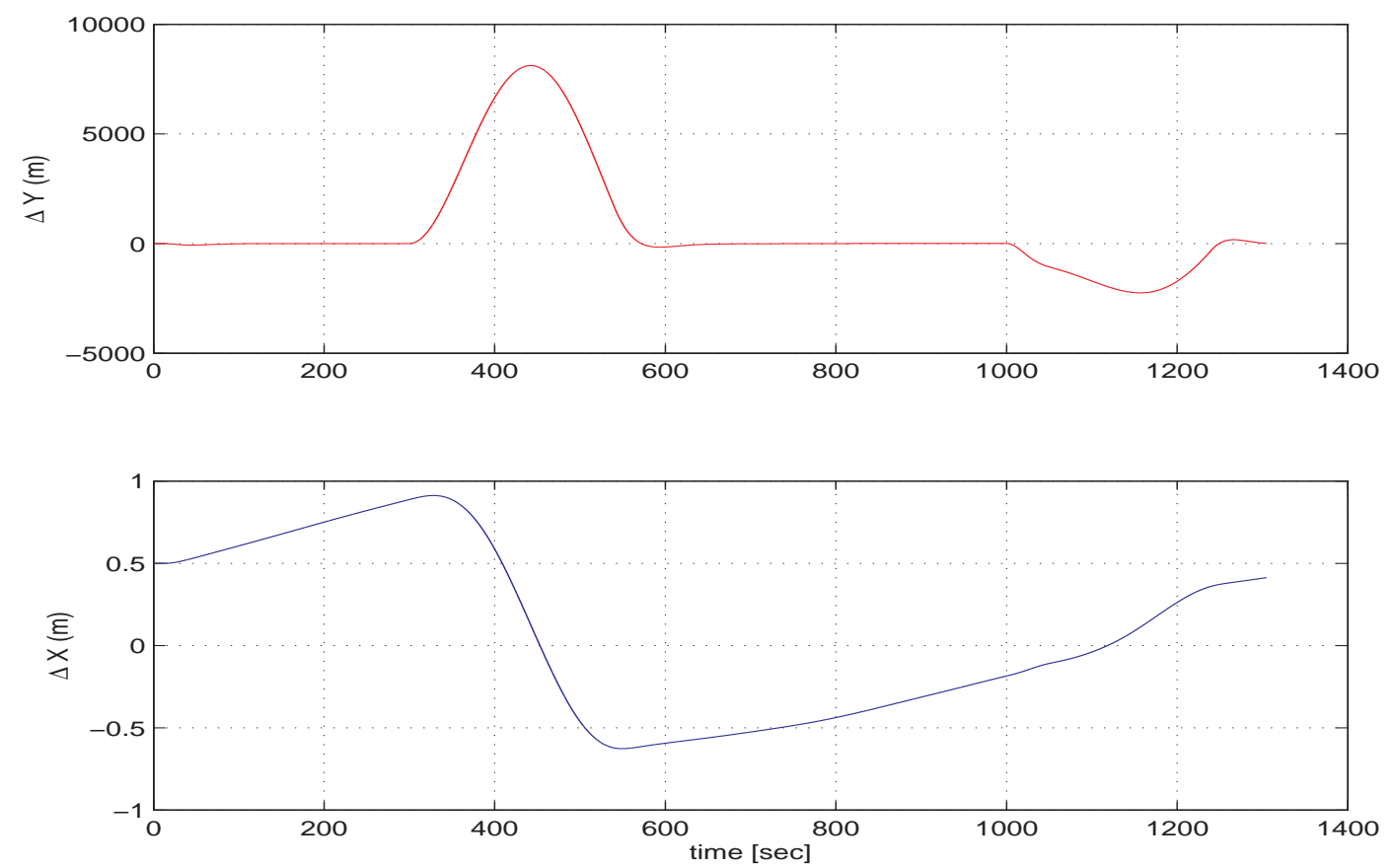

Figure 4.14. Relative position between tanker and virtual target.

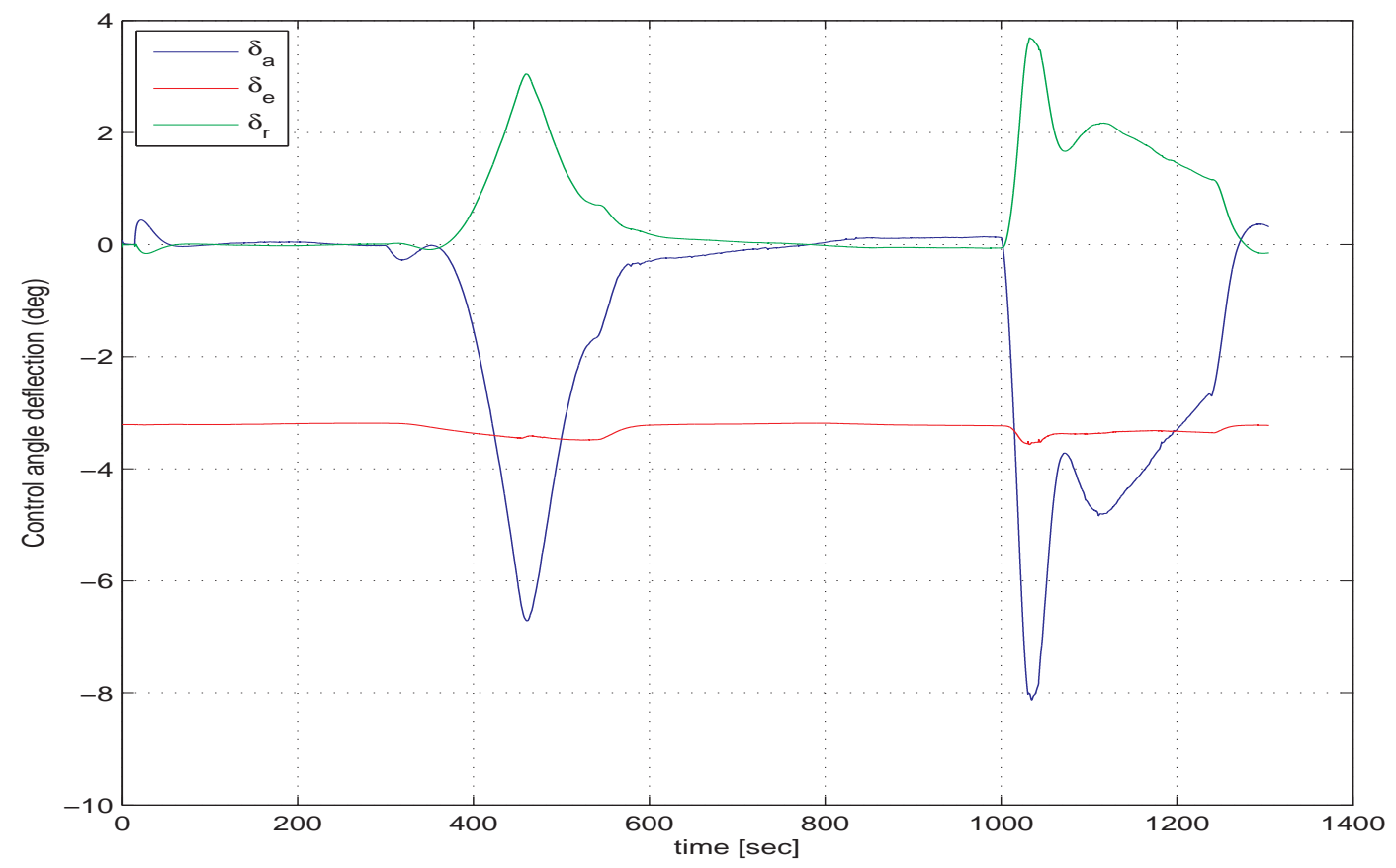

Figure 4.15. Control signals command. 


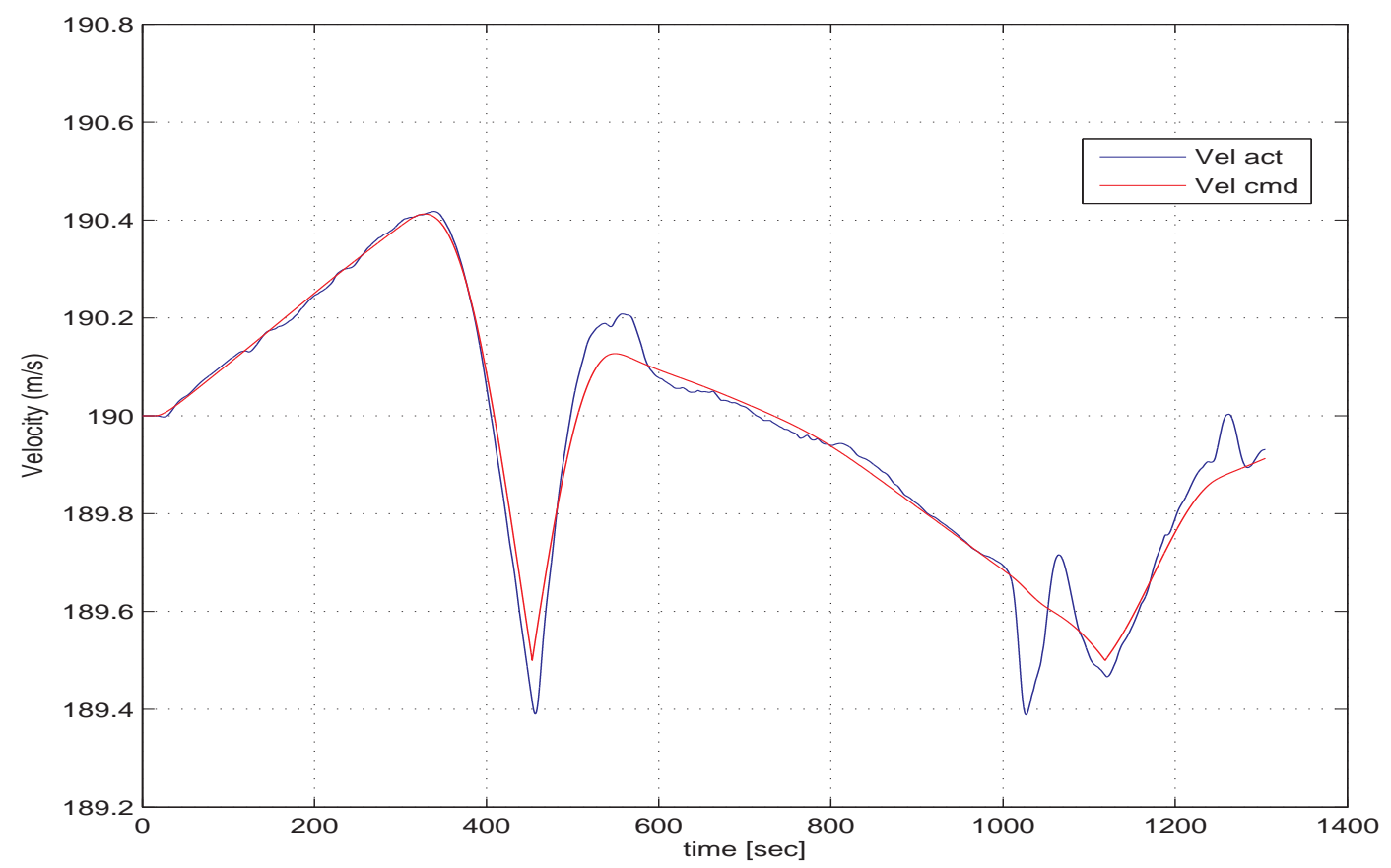

Figure 4.16. Commanded velocity versus actual velocity.

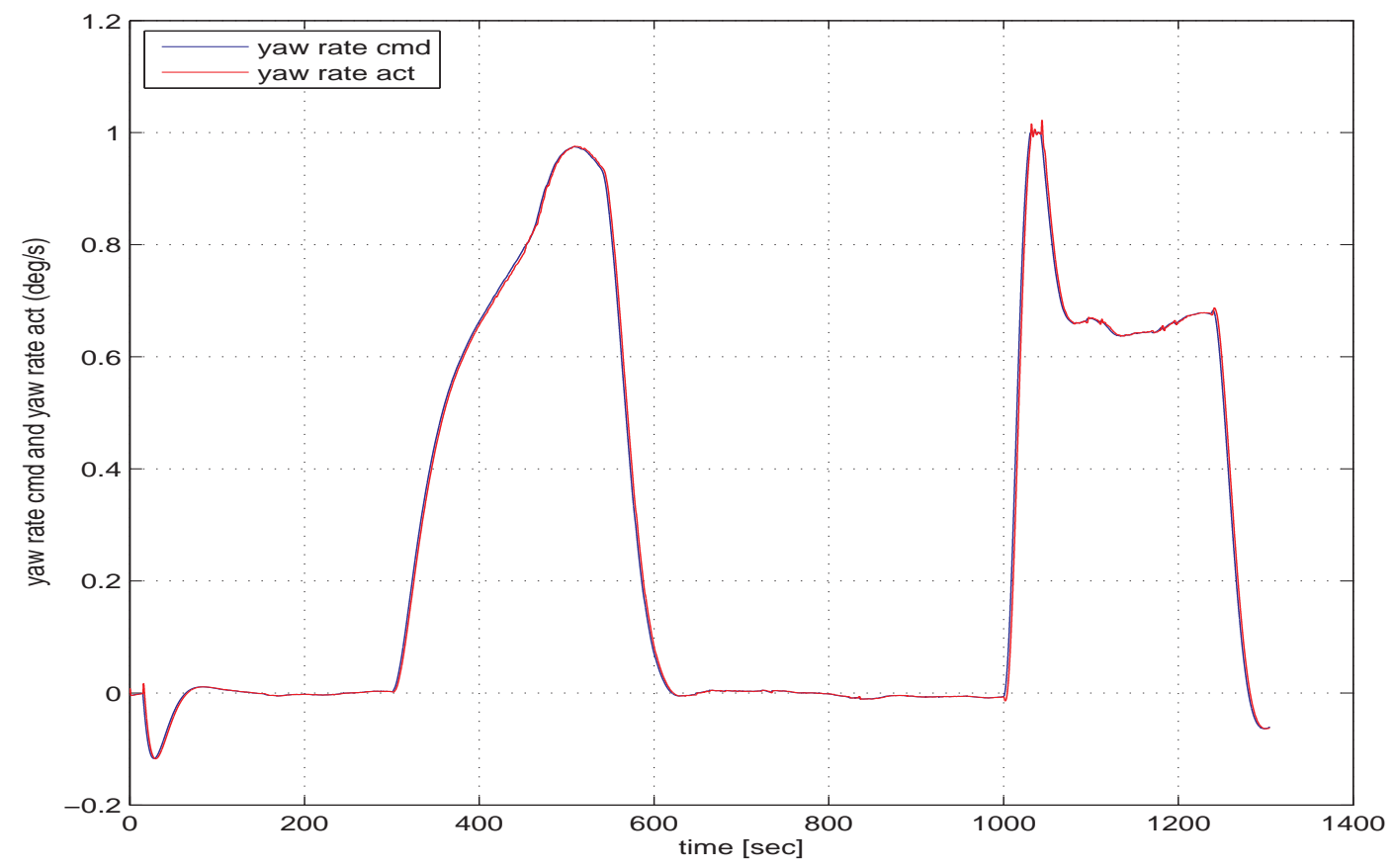

Figure 4.17. Commanded yaw rate versus actual yaw rate. 


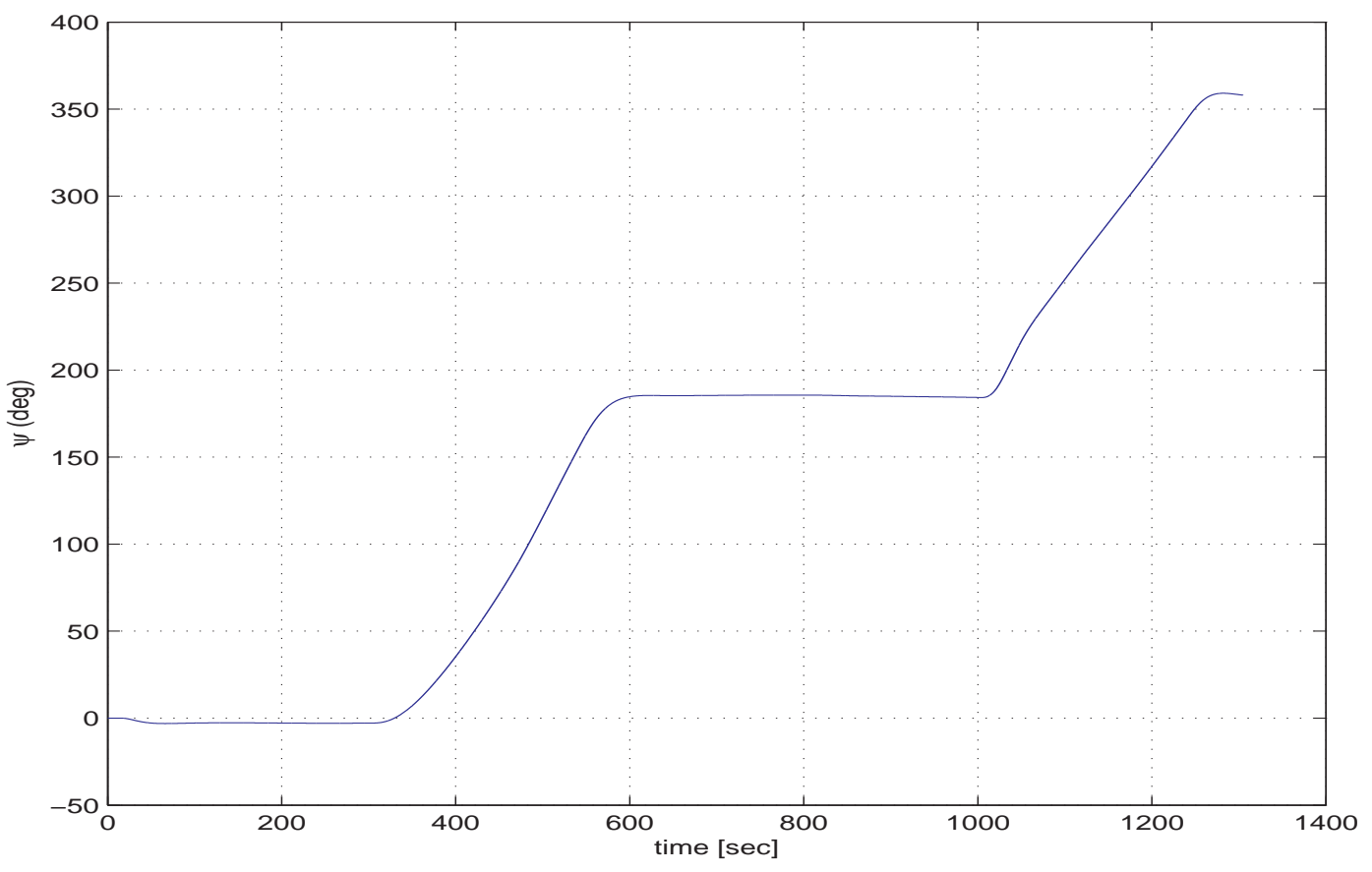

Figure 4.18. Yaw angle time history. 
4.3 Rendezvous of receiver aircraft with tanker

\subsubsection{Description of rendezvous simulation}

This section explains the simulation environment set up for the receiver aircraft. The primary task of the receiver aircraft is to rendezvous with the tanker aircraft at the ARCP while at the pre-contact position relative to the tanker in the presence of prevailing wind. Based on the modified point-parallel rendezvous procedure, the receiver aircraft flies along the aerial refueling line towards ARCP while the tanker moves on the refueling orbit. The synchronization of the receiver with the tankers achieved by following the virtual target moving along the aerial refueling line based on the motion of the actual tanker moving on the refueling orbit as detailed in Chapter 3.

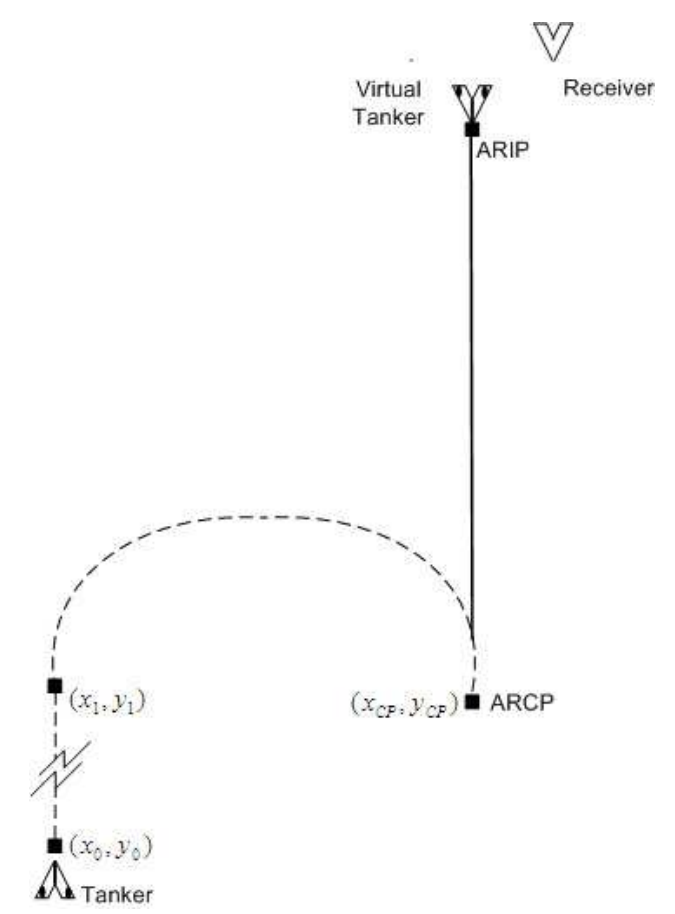

Figure 4.19. Rendezvous initial position of the aircrafts. 
In the simulation, the aerial refueling line runs South from ARIP to ARCP as depicted in Fig. 4.19. The tanker initially flies North on the straight leg of the refueling orbit and makes a 180-degree right turn to join the aerial fueling line at ARCP while flying South. The tanker is commanded to fly with nominal speed of $190 \mathrm{~m} / \mathrm{s}$, but the speed is adjusted by the proportional controller to regulate the distance with the virtual target. The receiver is commanded to stay at the precontract position of the virtual tanker, which is $(-59.13 m,-56.33 m, 0 m)$ with respect to the virtual tanker body frame, as depicted in, Fig. 4.19.

The tanker aircraft initially starts from the point $(0,0)$ with constant inertial airspeed of $190 \mathrm{~m} / \mathrm{s}$ and $\psi_{0}=0 \mathrm{deg}$ at altitude $7010 \mathrm{~m}$ and stays on the straight leg for $300 \mathrm{~s}$ and start turning to when reaches the point $\left(x_{1}, y_{1}\right)$ until at ARCP, which is the point $\left(x_{C P}, y_{C P}\right)$. In the meantime, the receiver aircraft flies form the initial position, which related to the initial position of the tanker aircraft which is ARIP, with inertial speed of $190 \mathrm{~m} / \mathrm{s}$ and yaw angle of $180 \mathrm{deg}$. The prevailing wind is turn on after 15 seconds. This rendezvous procedure is implemented in a MATLAB/Simulink environment with 6-DOF aircraft models including wind effect and the controllers for both aircraft. The functional block diagram of simulation environment is shown in Fig. 4.20. On the tanker side, the virtual target initially flies from point $(800 \mathrm{~m}, 0 \mathrm{~m})$ in the inertial frame, heading North with $\psi=0$ deg with constant speed $V=190 \mathrm{~m} / \mathrm{s}$ at $7010 \mathrm{~m}$ above mean sea level along the straight leg. The tanker aircraft initially flies from point $(0 \mathrm{~m}, 0 \mathrm{~m})$ in the inertial frame, heading North with $\psi=0 \mathrm{deg}$ with speed $V=190 \mathrm{~m} / \mathrm{s}$ at the same altitude as the virtual target aircraft. The tanker aircraft starts the simulation while $800 \mathrm{~m}$ right behind the virtual target. 


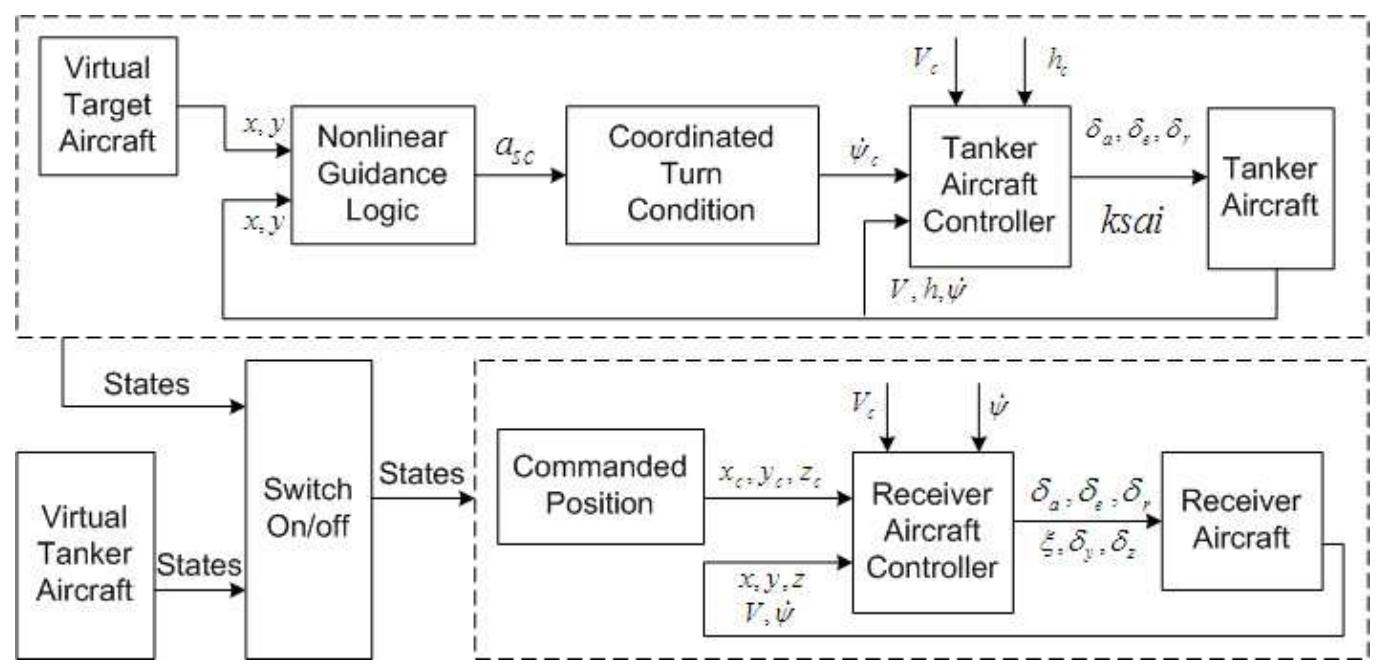

Figure 4.20. Block diagram for rendezvous simulation.

A time based logic is used to initiate the turn for the virtual target to change heading angle to $180 \mathrm{deg}$ from the current heading, 300 seconds. The turn rate of the virtual target is calculated from Eq. (3.6) to produce a lateral offset of 27,470 m.

The nonlinear guidance logic generates the lateral acceleration command to navigate the tanker aircraft along the desired trajectory which is generated by the virtual target. This acceleration command is then converted to the yaw rate command for the yaw rate controller to turn the tanker aircraft so that the lateral track or cross track error is reduced. while receiver follows the virtual tanker aircraft is used to output the tanker aircraft's states to the receiver aircraft. The tanker state values needed in the receiver model are set to nominal values of the actual tanker aircraft to minimize the effect of the transition when the receiver switches to following the actual aircraft after ARCP.

For first order filters are employed to reduce the effect of the difference between the states of the virtual tanker and the actual tanker before transition form the virtual tanker to the actual tanker for the receiver to follow. These filters are needed especially for dominant states such as $\dot{x}, \dot{y}, \psi$ and $\phi$. 
A switching logic is used for switching from the virtual tanker to the tanker aircraft at the specified time for the receiver to keep staying at the pre-contract position.

For the receiver aircraft trajectory tracking controller, constant position commands are given. The position commands correspond to the precontract position $(-59.13 m,-56.33 m, 0 m)$. This is to fly the receiver at the precontract position with respect to the virtual tanker so that when transitioned to following the actual tanker aircraft, the receiver will be close to the precontract position of the tanker aircraft.

In the receiver aircraft equation of motions, the translational kinematics equations which is modeled relative to the tanker aircraft's body frame is modified to handle the relative position jump when switching from the virtual tanker to the tanker aircraft by recalculating the relative position of the receiver aircraft during the jump. The position of the receiver relative to the tanker aircraft is generated by the relative translational kinematics in Eq. (2.10). This equation formulates the effect of the tanker aircraft translational and rotational velocity on the relative position. Its integration computes the relative position as the tanker position and orientation change continuously based on the corresponding velocity vectors. However, during the time of the receiver switching from the virtual tanker to the actual tanker, there is an instantaneous jump in the position of the reference frame that the receiver relative position is defined in. To overcome this problem, the relative position integration state is reset during the time of the switch. The reset value is calculated as follows. The receiver position relative to the inertial frame is the vectorial sum of the position of the tanker and the relative position.

$$
\underline{\mathrm{r}}_{R}=\underline{\mathrm{r}}_{T}+\underline{\xi}
$$


which gives the relative position vector as

$$
\underline{\xi}=\underline{\mathrm{r}}_{R}-\underline{\mathrm{r}}_{T}
$$

when written in terms of the representation of the vectors, Eq. (4.3.1) becomes

$$
r_{R}=r_{T}+\mathbf{R}_{B_{T}, I}^{T} \xi
$$

where $r_{R}$ is the representation in the inertial frame of the position of the receiver relative to the inertial frame, $r_{T}$ is that of the tanker, $\xi$ is the representation in the tanker's body frame of the position vector of the receiver relative to the tanker and $\mathbf{R}_{\mathbf{B}_{\mathbf{T}}, \mathbf{I}}$ is the rotation matrix from the inertial frame to tanker's body frame. When the receiver follows the virtual tanker, $r_{T}$ in Eq. (4.3.1) is the position of the virtual tanker.

When the receiver switches to following the actual tanker, $r_{T}$ in Eq. (4.3.1) will also switch from the position of the virtual tanker to the position of the actual tanker. When the positions of the virtual and actual tanker are adopt, Eq. (4.3.1) will result in a discontinuity in $r_{R}$ which is not realistic since $r_{R}$ is the physical position of the actual receiver aircraft. Instead, the relative position $\xi$ should have the corresponding discontinuity. To achieve this, the position of the receiver relative to the inertial frame is calculated based on the tanker position and the relative position as

$$
r_{R}=r_{T^{-}}+\mathbf{R}_{\mathbf{B}_{\mathbf{T}}, \mathbf{I}}^{\mathbf{T}} \xi^{-}
$$

where $r_{T^{-}}$and $\xi^{-}$represent tanker position and relative position right before the jump. After the jump, the position of the tanker will change to $r_{T^{+}}$if the position of the actual tanker and the virtual tanker are not the same. Using the receiver position $r_{R}$ and tanker position $r_{T^{+}}$, the relative position after the jump is calculated as

$$
\xi^{+}=\mathbf{R}_{\mathbf{B}_{\mathbf{T}}, \mathbf{I}}\left(r_{R}-r_{T}^{+}\right)
$$

which is used to reset the integral state of the translational kinematics of the receiver. 


\subsubsection{No wind condition}

The performance of the receiver aircraft rendezvous procedure is evaluated first without any prevailing wind. This also done to obtain a base line case to compare the simulations results with prevailing wind. Fig. 4.21 shows the trajectories in the

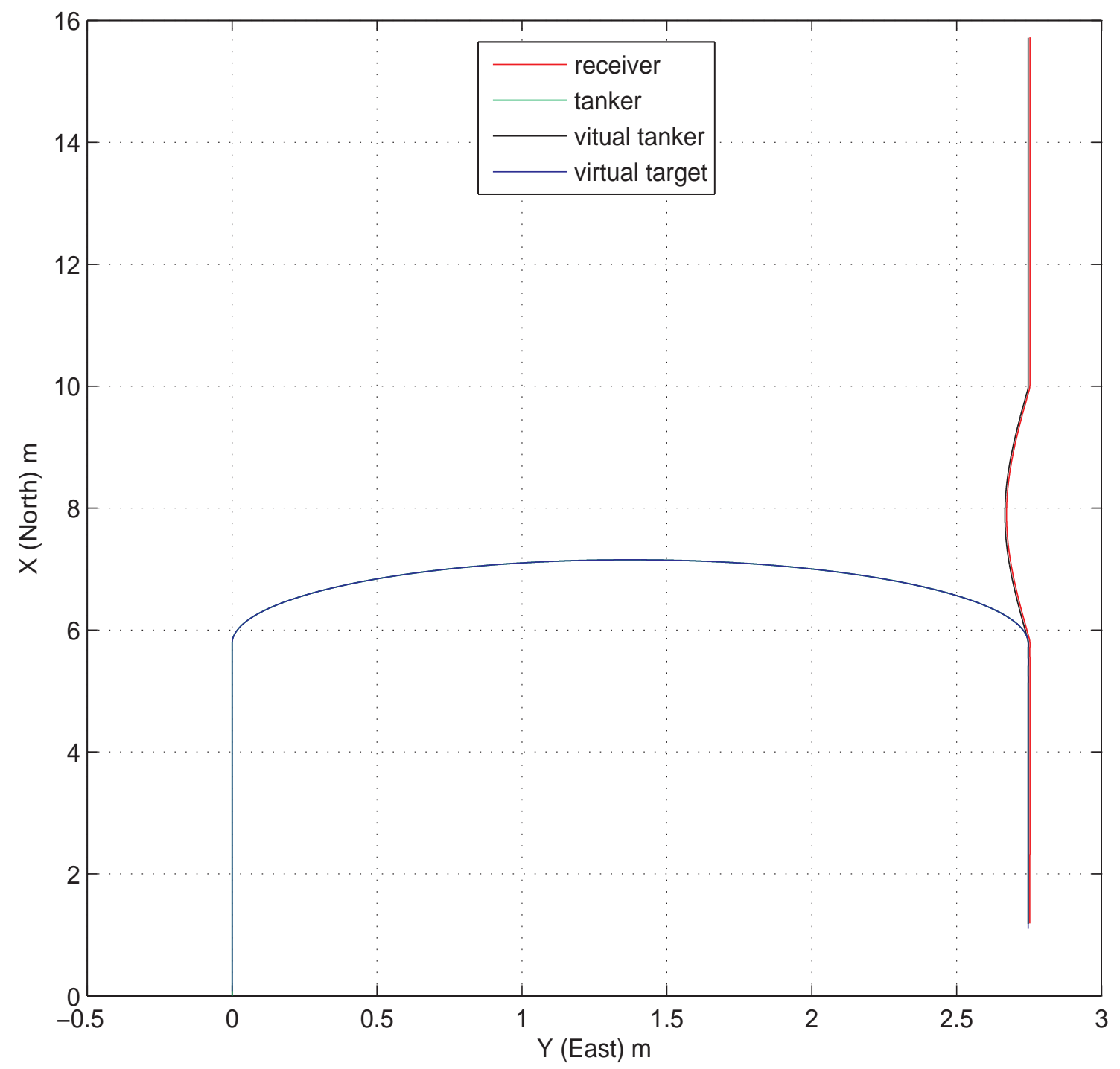

Figure 4.21. Trajectory comparison of the aircrafts. 
inertial frame of the virtual target, the tanker aircraft, the receiver aircraft and the virtual tanker. In the scale of the figure, the differences between the tanker and the virtual target, and between the receiver and the virtual tanker are not visible. The receiver success fully rendezvous with the tanker at ARCP. An interesting observation is the straight west wand deviation of the virtual tanker and the receiver from the aerial refueling line. This is because of the deviation of the tanker from the virtual target trajectory during turn. The guidance algorithm implemented for the virtual tanker deviates its trajectory from the aerial refueling line to synchronize its arrival at ARCP with the tanker. The comparison of the virtual target's and the tanker
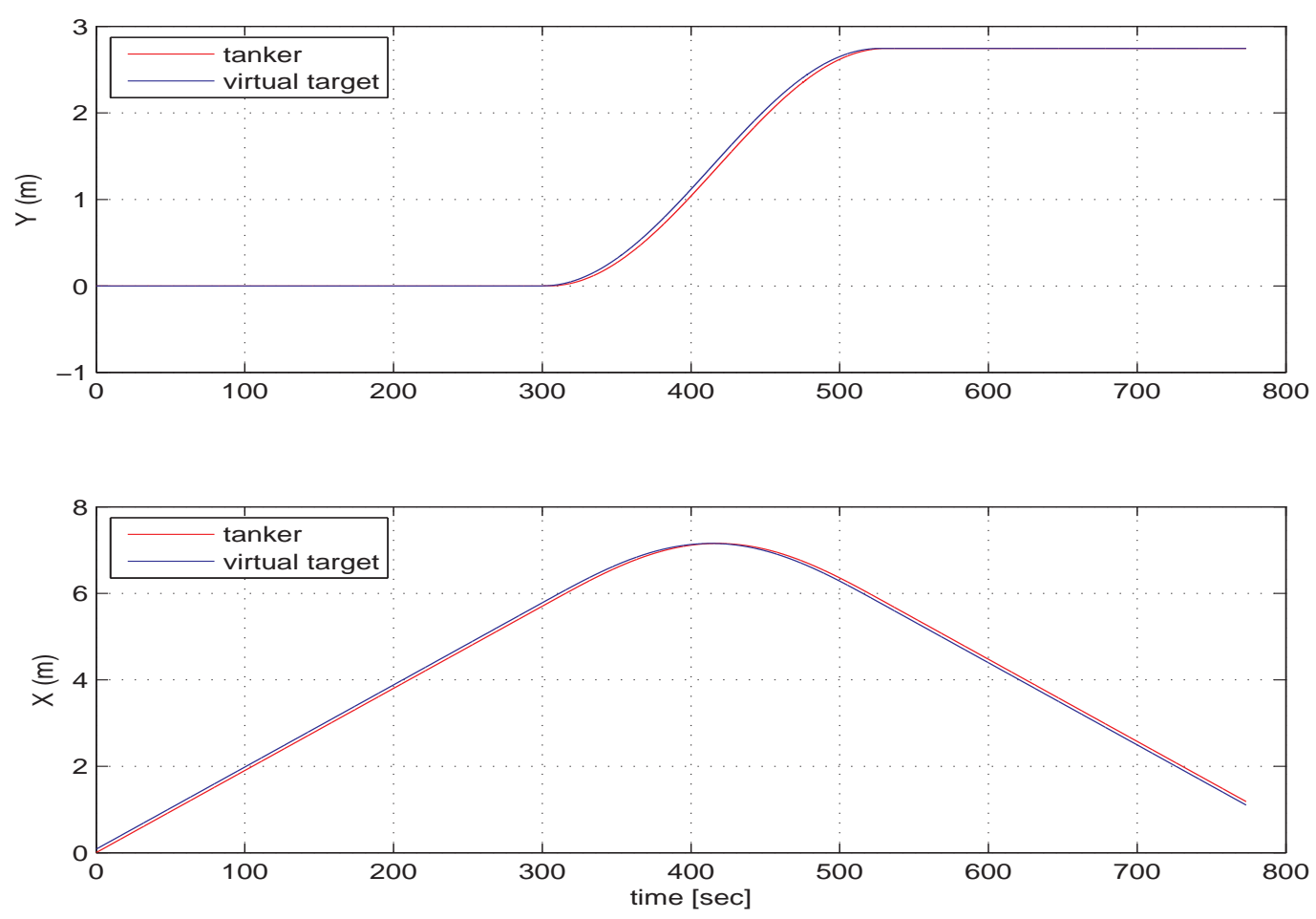

Figure 4.22. Tanker versus virtual target trajectory in $\mathrm{x}-\mathrm{y}$ axis.

aircraft's position in $\mathrm{x}$ and $\mathrm{y}$ directions is shown in Fig. 4.22. The relative position between the tanker and the virtual target in the $\mathrm{x}$ and $\mathrm{y}$ axis is also shown in Fig. 4.23. 
The results shown that the tanker aircraft is capable of tracking the virtual target perfectly. Note that in Fig. 4.23 the difference along $\mathrm{x}$ and $\mathrm{y}$-axis are constant, where $\triangle x=800 m$ and $\triangle y=0 m$ before and after the turn. The comparison of
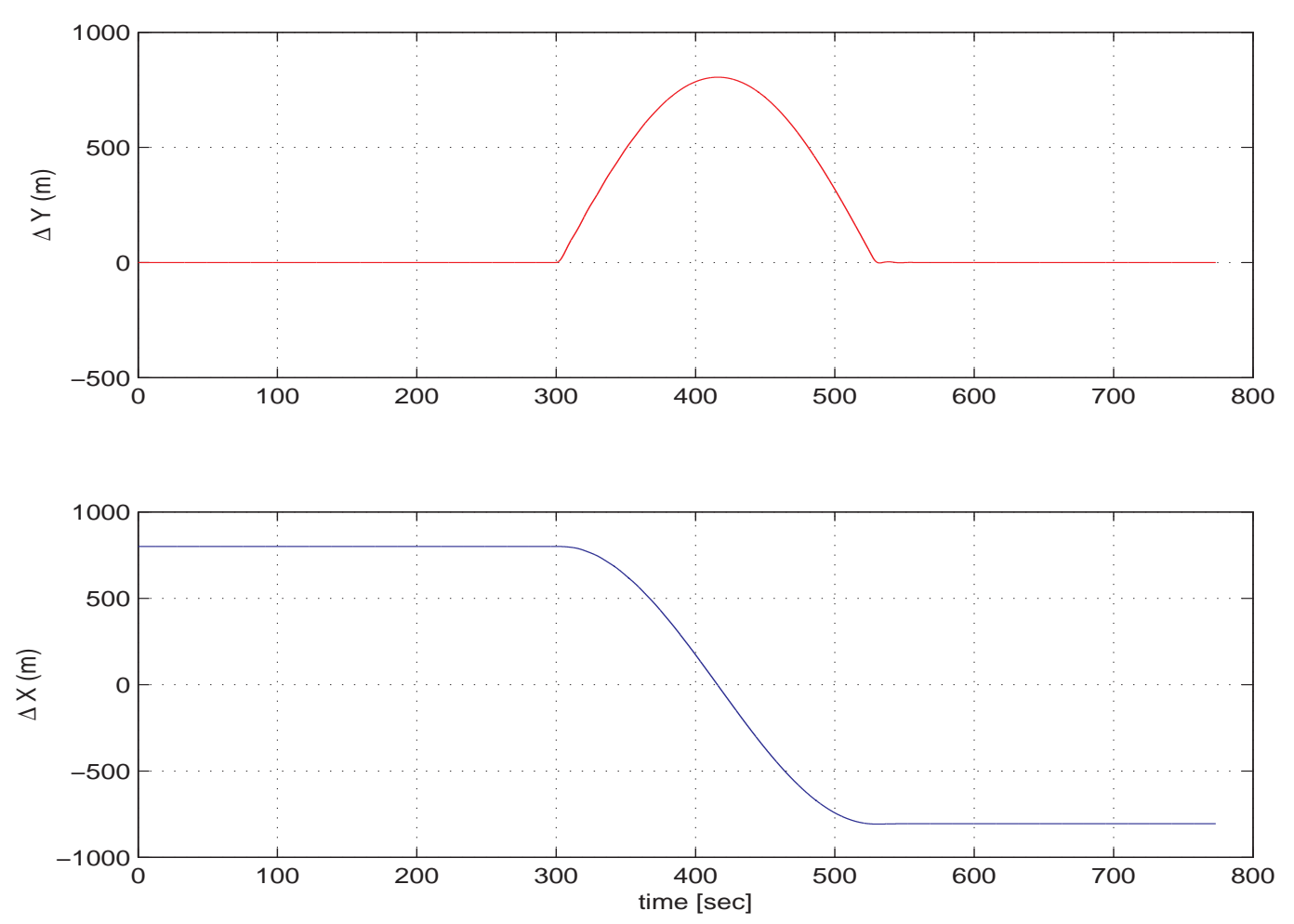

Figure 4.23. Tanker versus virtual target relative position along $\mathrm{x}-\mathrm{y}$ axis.

the tanker's and the virtual tanker aircraft's position in $\mathrm{x}$ and $\mathrm{y}$ direction is shown in Fig. 4.24. The tanker aircraft and the virtual tanker aircraft rendezvous at time $527 s$ which is the time when the virtual target stop turning. The tanker aircraft inertial velocities is shown in Fig. 4.25. The airspeed in $\mathrm{x}$ and $\mathrm{y}$-axis are smooth. The airspeed in the z-axis had some small variation about $\pm 0.3 \mathrm{~m} / \mathrm{s}$ when the tanker starts turning and rolling out. The receiver aircraft inertial velocities components as shown in Fig. 4.26 are all constant until switching from the virtual tanker to the tanker aircraft. The tanker's aircraft orientation relative to the inertial frame is 

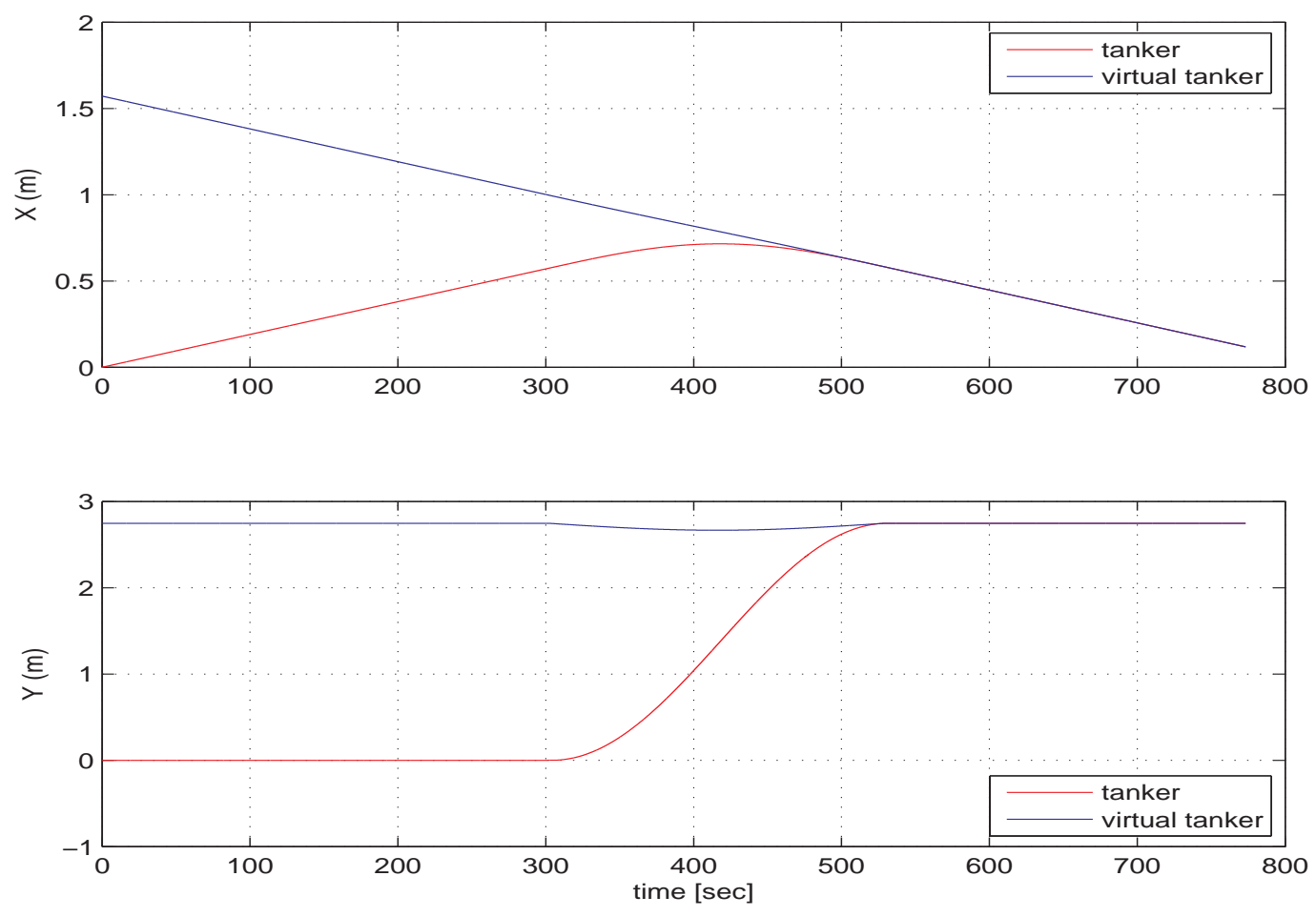

Figure 4.24. Tanker versus virtual tanker position trajectory in $\mathrm{x}-\mathrm{y}$ axis.

shown in Fig. 4.27. The results shown that, the orientation of the tanker aircraft demonstrates the standard characteristics of a straight and level flight and 180-deg turn. The yaw angle is 0 degree until starts turning and the total change in heading is 180 degrees when the turn is completed. The bank angle during the turn is 16 degrees. However, it shows a transient at the beginning and end of the turn. The receiver aircraft's orientation relative to the tanker frame is shown in Fig. 4.28. The results show that, the receiver aircraft maintains a constant orientation relative to the virtual tanker all the time. Only transient occurs after switching to the actual tanker. The tanker aircraft's control surface deflections and throttle setting are shown in Fig. 4.29. The results show that the control surface deflections of the tanker aircraft are small during the whole racetrack maneuver. Similarly, the throttle setting is slightly employed during the start and end of the turns. The receiver 

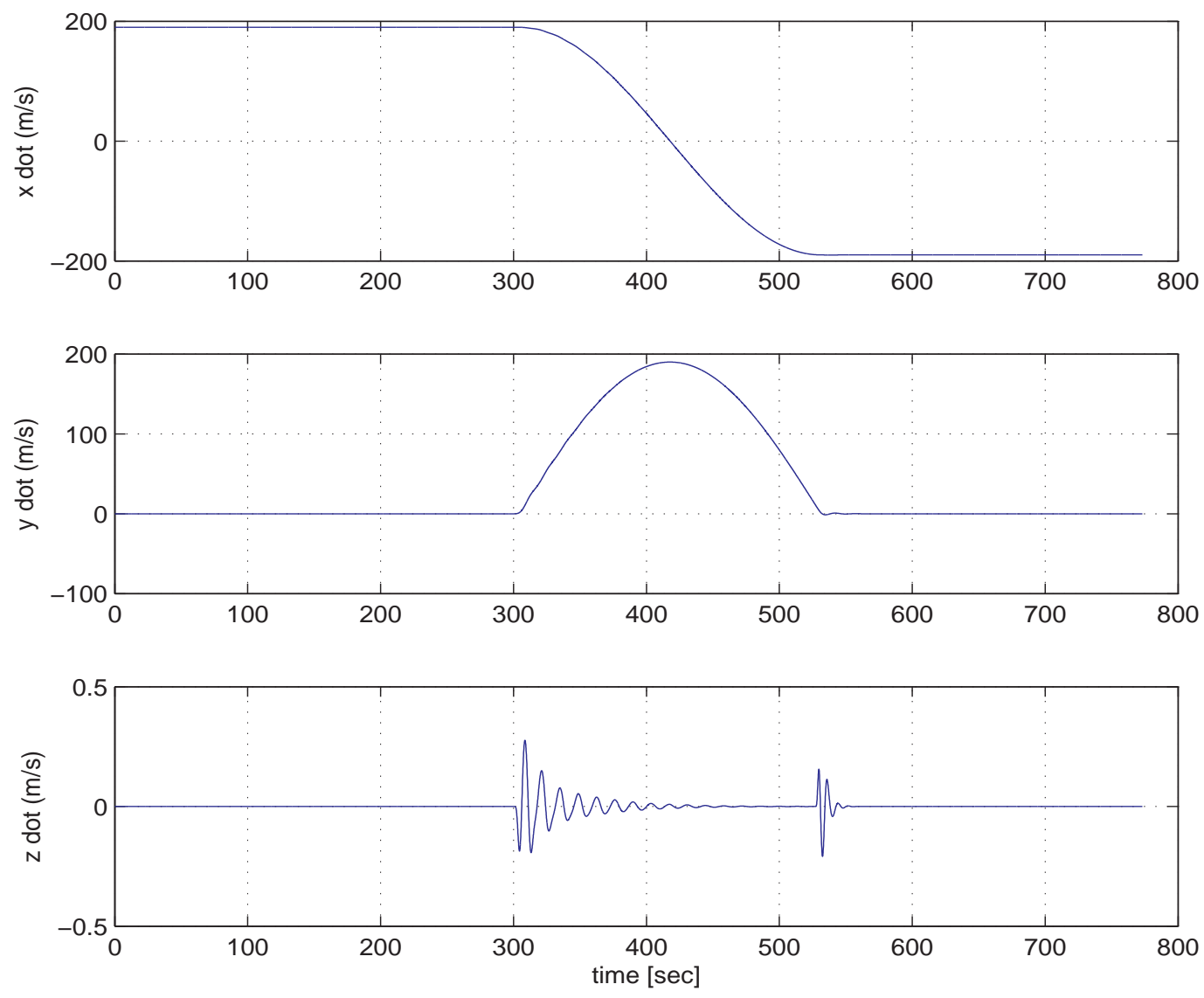

Figure 4.25. Tanker aircraft inertial velocities.

aircraft control efforts are shown in Fig. 4.30. The results show also that the control surface deflections of the receiver aircraft are also very small with small transient when switching form the virtual tanker to the tanker aircraft. The receiver adjusts its airspeed by small throttle action when switching from the virtual tanker to the actual tanker. The receiver aircraft's relative position is shown in Fig. 4.31. The result show that the receiver aircraft is capable of tracking the virtual and actual tanker with a good performance. Note that the commanded position is shifted to the position of the receiver relative to the actual tanker once the receiver is switched to following the actual tanker. This is to avoid the discontinuity in the relative position error. Once the discontinuity is handled this way, the commanded position 

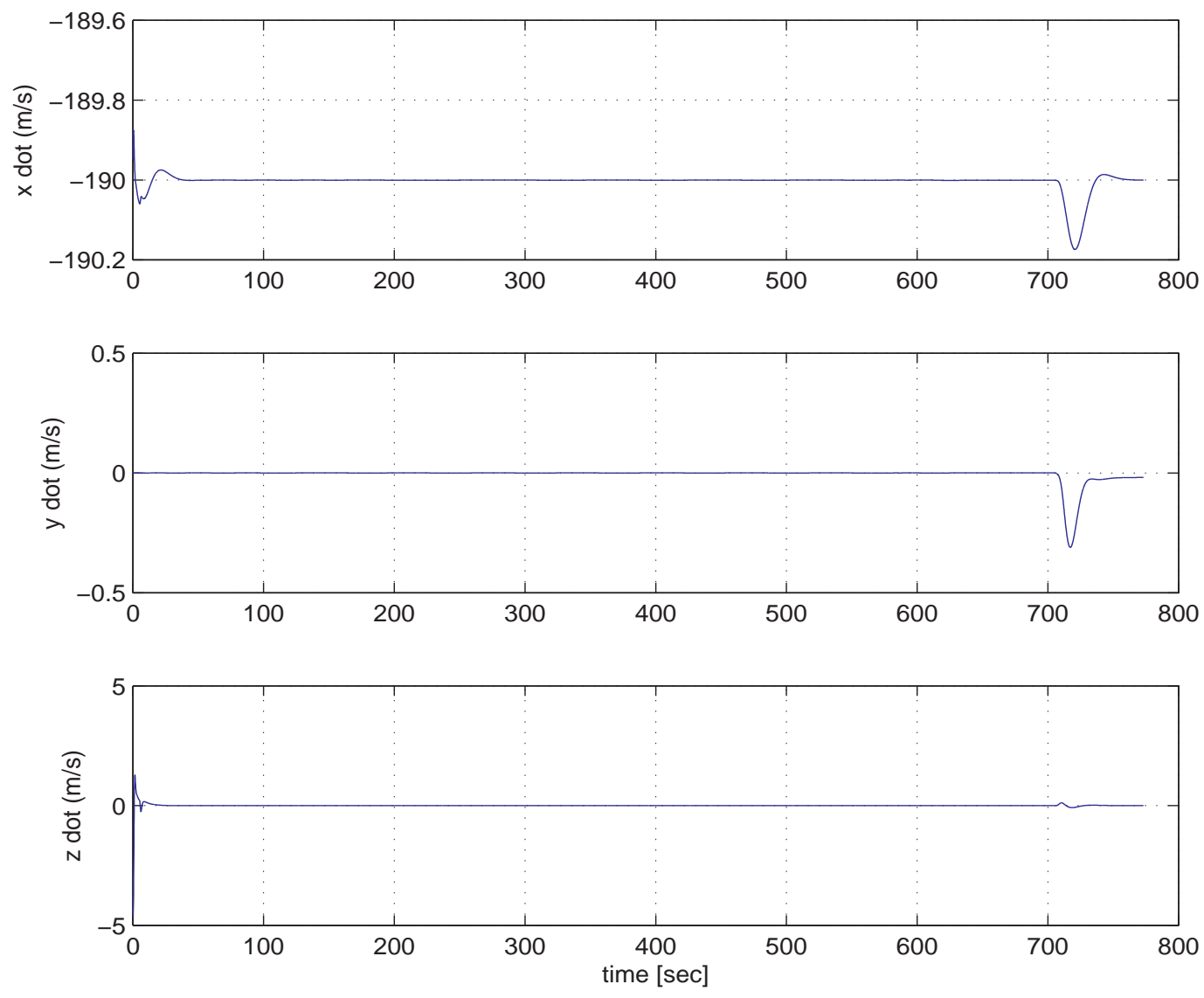

Figure 4.26. Receiver aircraft inertial velocities.

is gradually changed back to the pre-contract position to move the receiver to the precontract position of the actual tanker in a controlled fashion. 

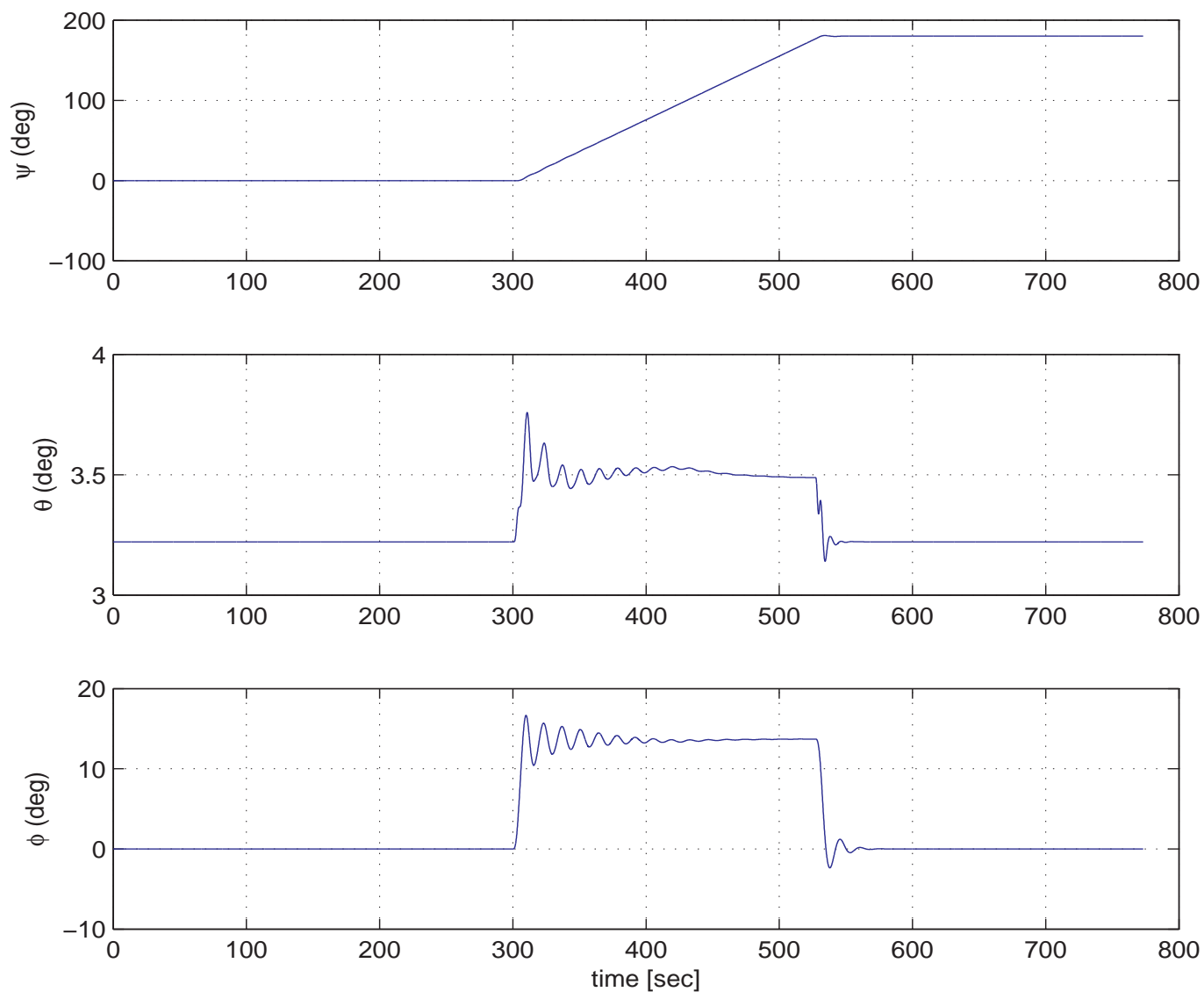

Figure 4.27. Tanker aircraft Euler's angle. 

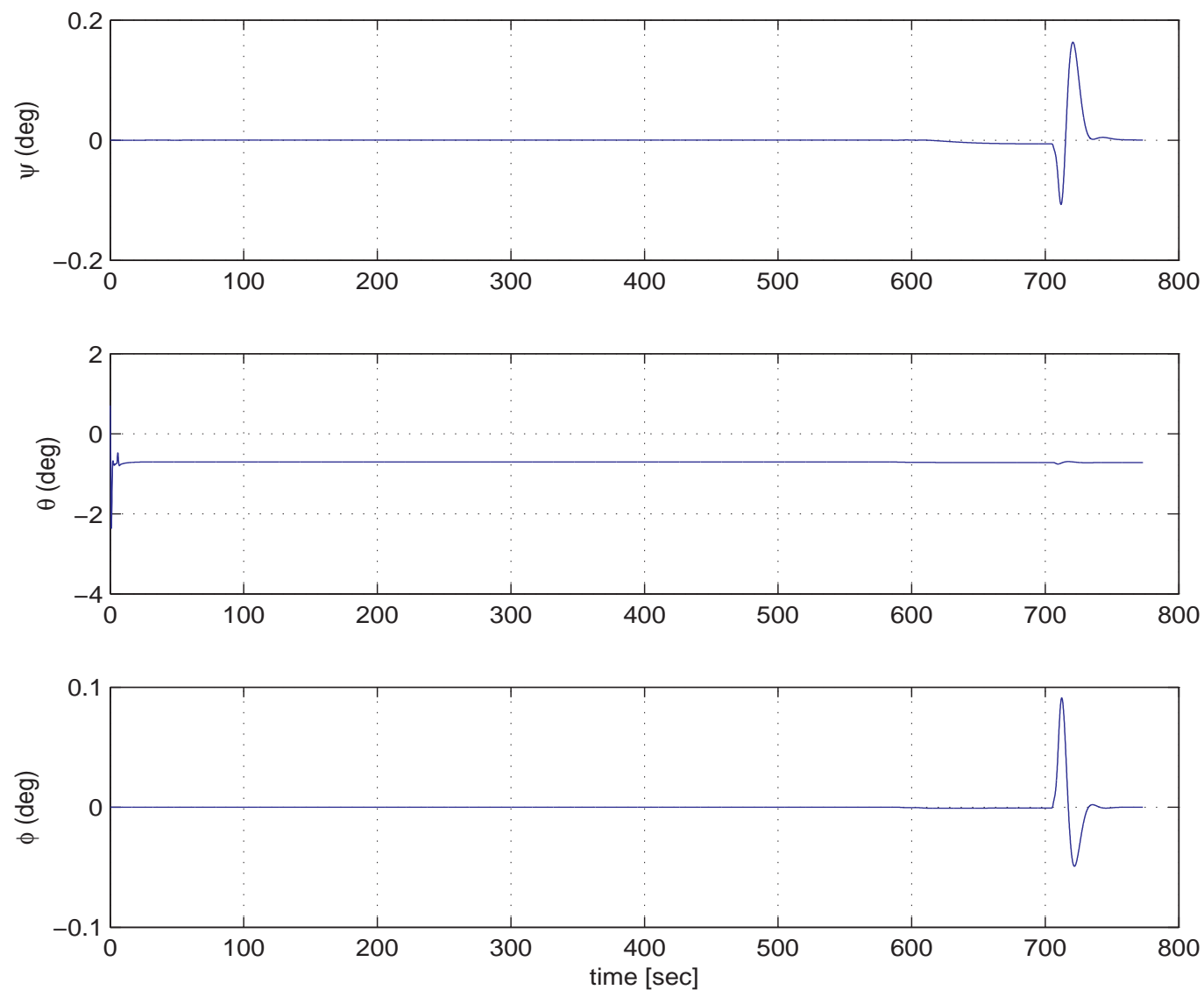

Figure 4.28. Receiver aircraft Euler's angle. 

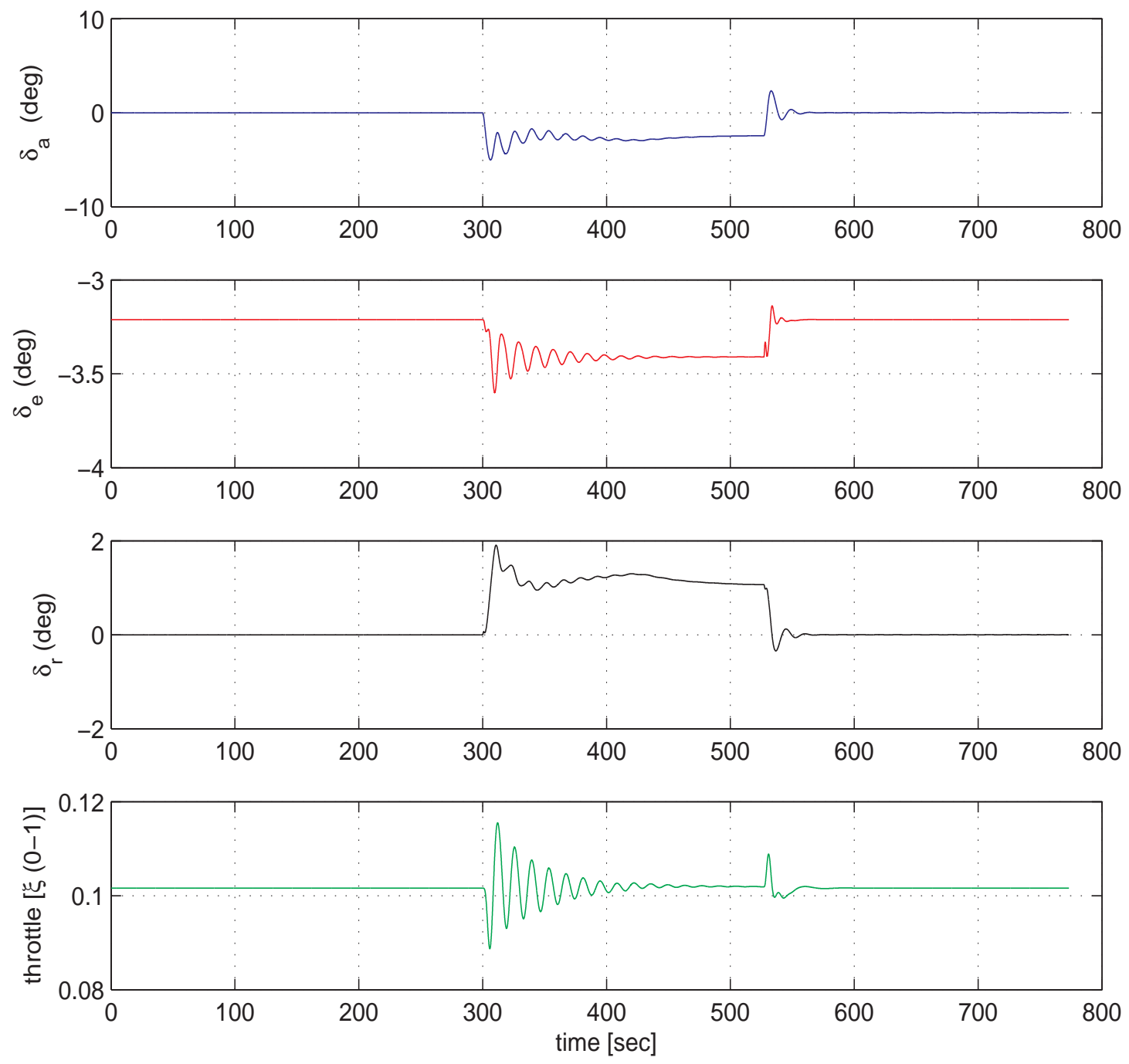

Figure 4.29. Tanker aircraft control signals. 

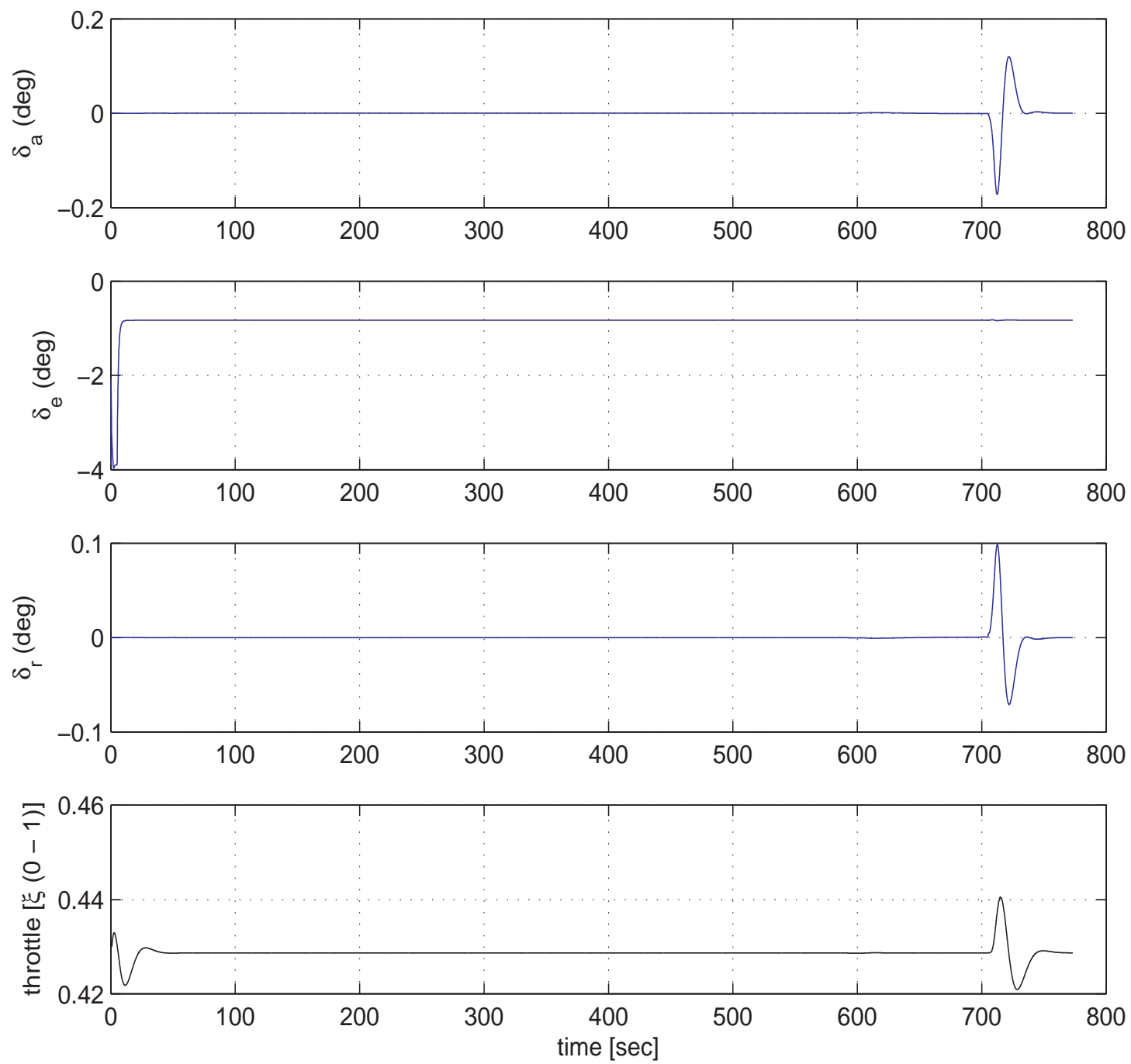

Figure 4.30. Receiver aircraft control signals. 

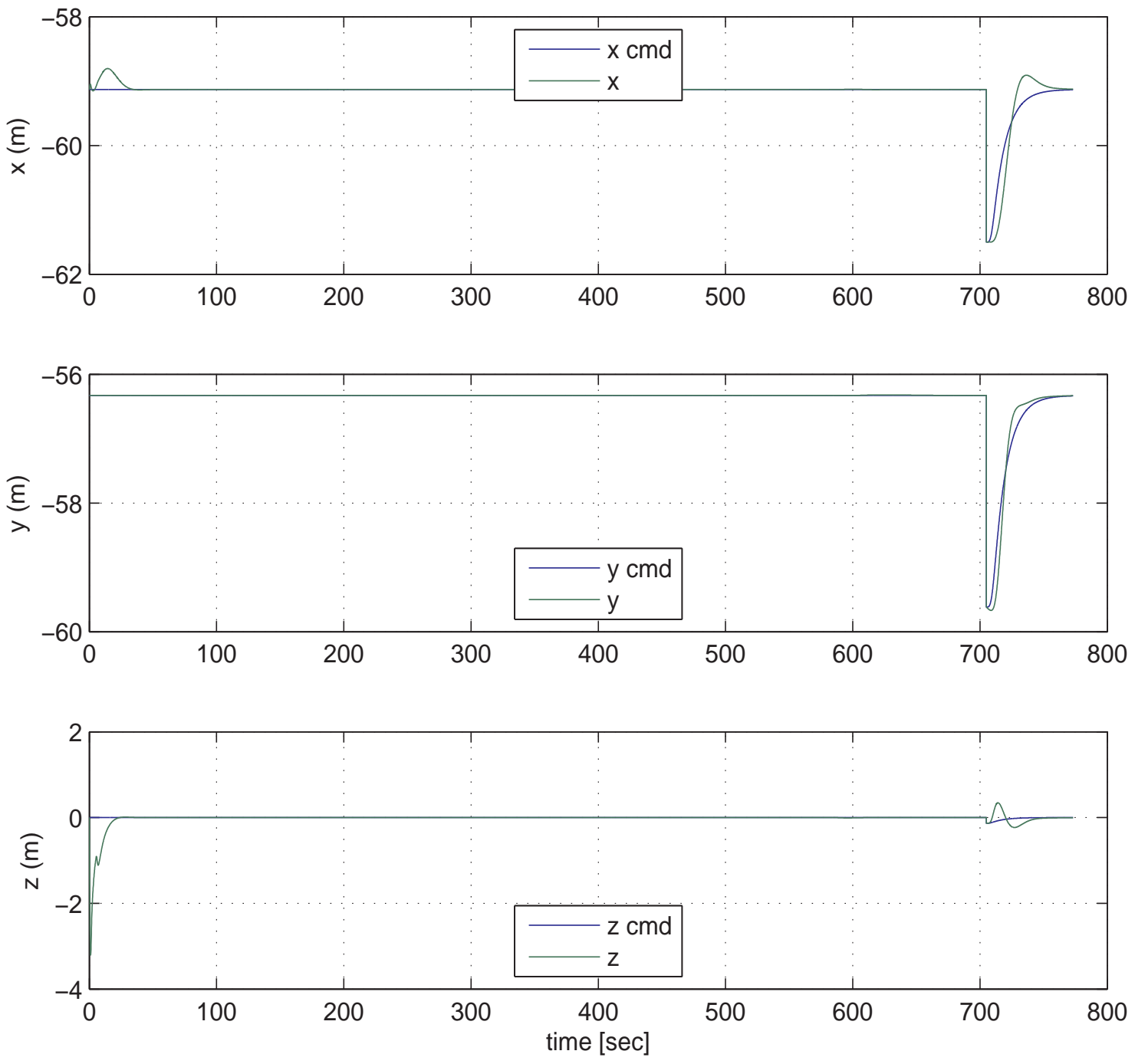

Figure 4.31. Receiver aircraft's relative position. 


\subsubsection{Wind condition}

This section presents the simulation of the rendezvous in the presence of a realistic prevailing wind contribution, as described in Fig. 4.9 in section 4.2.2. As stated there, the prevailing wind blows in Southeast direction. In the simulation, the wind is gradually turned on starting at 15 seconds. This simulation should demonstrates the performance of the receiver rendezvous guidance and control capability in the presence of wind. The effect of the wind is two-folded. The wind disturbs the tanker and degrades its tracking performance of the virtual target. This means the receiver needs to synchronize its motion with a tanker that does not fly on a perfect refueling orbit. The other effect of wind is direct on the dynamics and control of the receiver aircraft. Fig. 4.32 shows the trajectories in the inertial frame of the virtual target, the tanker aircraft, the receiver aircraft and the virtual tanker aircraft. The virtual tanker trajectory computed by the guidance algorithm in chapter 3 first has a straight line and then deviates from the aerial refueling line through a bell-shaped curved towards west. This is because the prevailing wind degrades the nonlinear guidance performance of the tanker aircraft during the start and end of the turn, which result in large deviation of tanker trajectory from the virtual target trajectory, which represents the perfect aerial refueling orbit. This delays arrival of the tanker aircraft at ARCP because the distance-to-travel is increased. The guidance algorithm of the receiver nicely adjusts the trajectory of the receiver to ensure its timely arrival at the rendezvous point.

The comparison of the virtual target's and the tanker aircraft's position in $\mathrm{x}$ and $y$ direction is shown in Fig. 4.33. The tanker aircraft is capable of tracking the desired trajectory in the y-direction as concluded by the work in Ref [1]. This observation can be more clearly seen in Fig. 4.34. Note that because of the prevailing wind, the tanker has difficulty maintaining its longitudinal distance with the virtual 


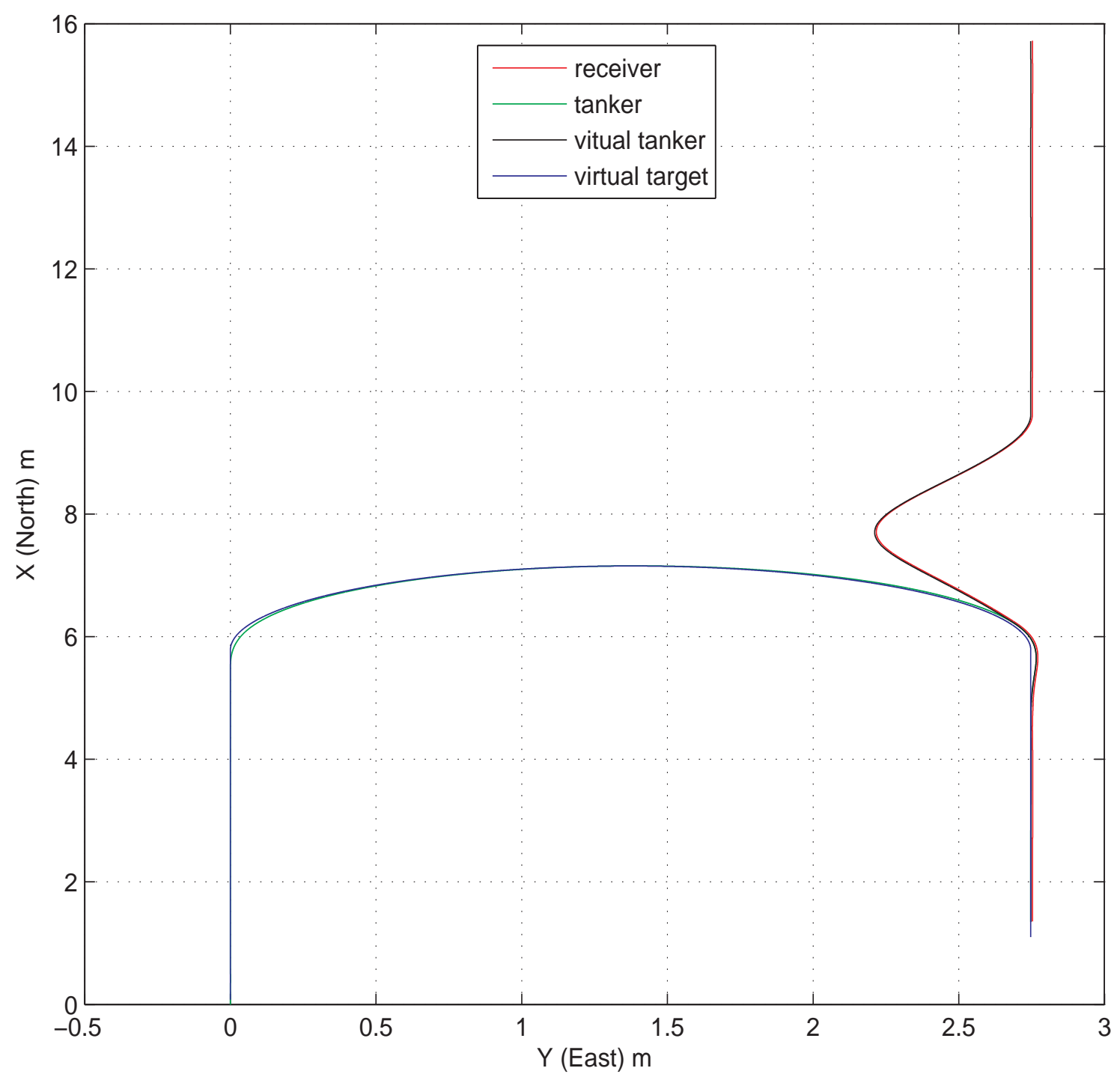

Figure 4.32. Trajectory comparison of the aircrafts.

target. When the prevailing wind acts as headwind, the ground speed of the tanker drops and as a result the distance increases.

The comparison of the tanker's and the virtual tanker aircraft's position in $\mathrm{x}$ and $y$ direction is shown in Fig. 4.35. The tanker aircraft and the virtual tanker 

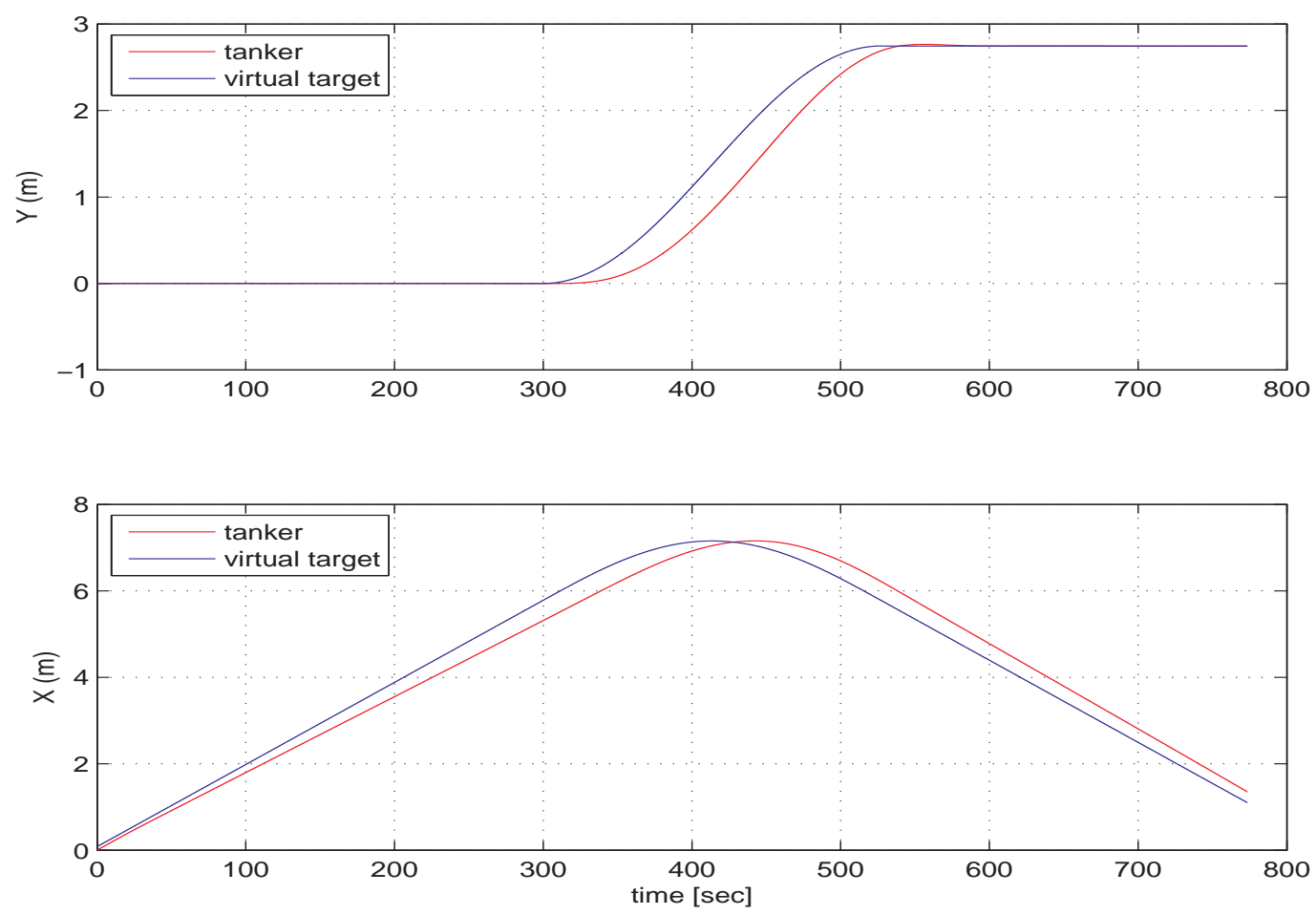

Figure 4.33. Tanker versus virtual target trajectory in $\mathrm{x}-\mathrm{y}$ axis.

aircraft rendezvous at time $527 \mathrm{~s}$, which is the time when the virtual target stops turning. The tanker aircraft inertial velocity components are shown in Fig. 4.36.

The receiver aircraft inertial velocity components are shown in Fig. 4.37. The velocity components are constant when the virtual target is followed but start fluctuating when the actual tanker is tracked. This is because the velocity of the actual tanker fluctuates due to the prevailing wind.

The tanker's aircraft orientation relative to the inertial frame is shown in Fig. 4.38. The tanker yaw angle initially is 0 degree until the time $15 \mathrm{~s}$ when the prevailing wind is turned on. The tanker controller responds to wind encounter by changing the yaw angle -2.7 degrees until the virtual target starts turning. The tanker yaws to the left because it tries to keep flying along the straight leg of the 

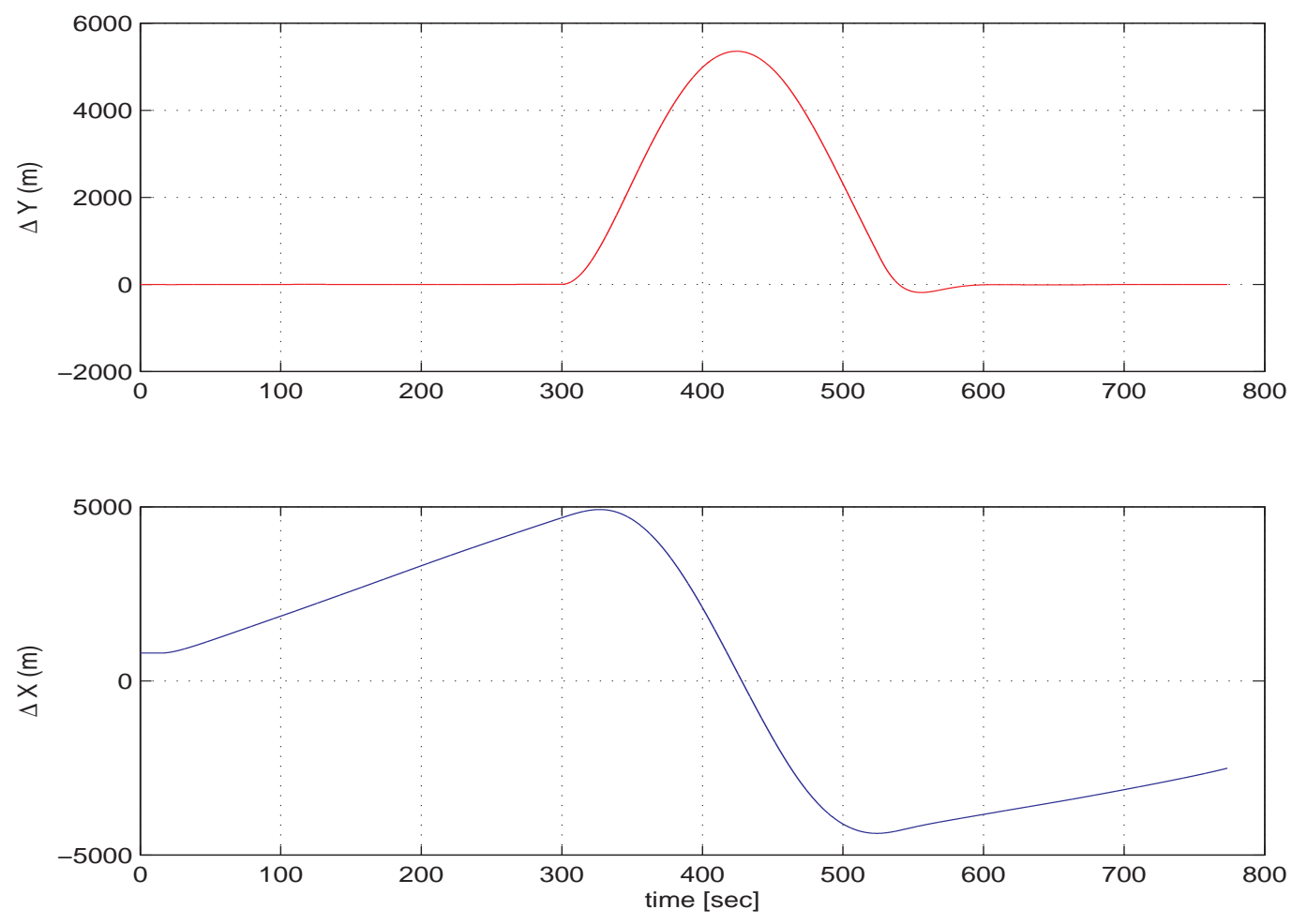

Figure 4.34. Tanker versus virtual target position difference along x-y axis.

refueling track. The effects of the wind in pitch and roll are also displayed by the transients which last for $20 \mathrm{~s}$.

During the turn, the pitch angle of the tanker is increased until the halfway of the turn and then starts decreasing because when the tanker's bank angle increases, the amount of lift starts decreasing. To maintain the coordinated turn with constant altitude, the tanker increases its angle of attack and also the pitch angle. During the rendezvous turn, the bank angle is about 15 degrees. The receiver's aircraft orientation relative to the tanker frame is shown in Fig. 4.39. The wind effect on the Euler's angle can be seen. When the receiver aircraft encounters the tailwind and blowing from Southeast, the receiver aircraft yaws to the right to keep tracking the straight line and stay at the pre-contract position. Fig. 4.39 also shows the transients that happen when switching from the virtual tanker aircraft to the tanker aircraft. 

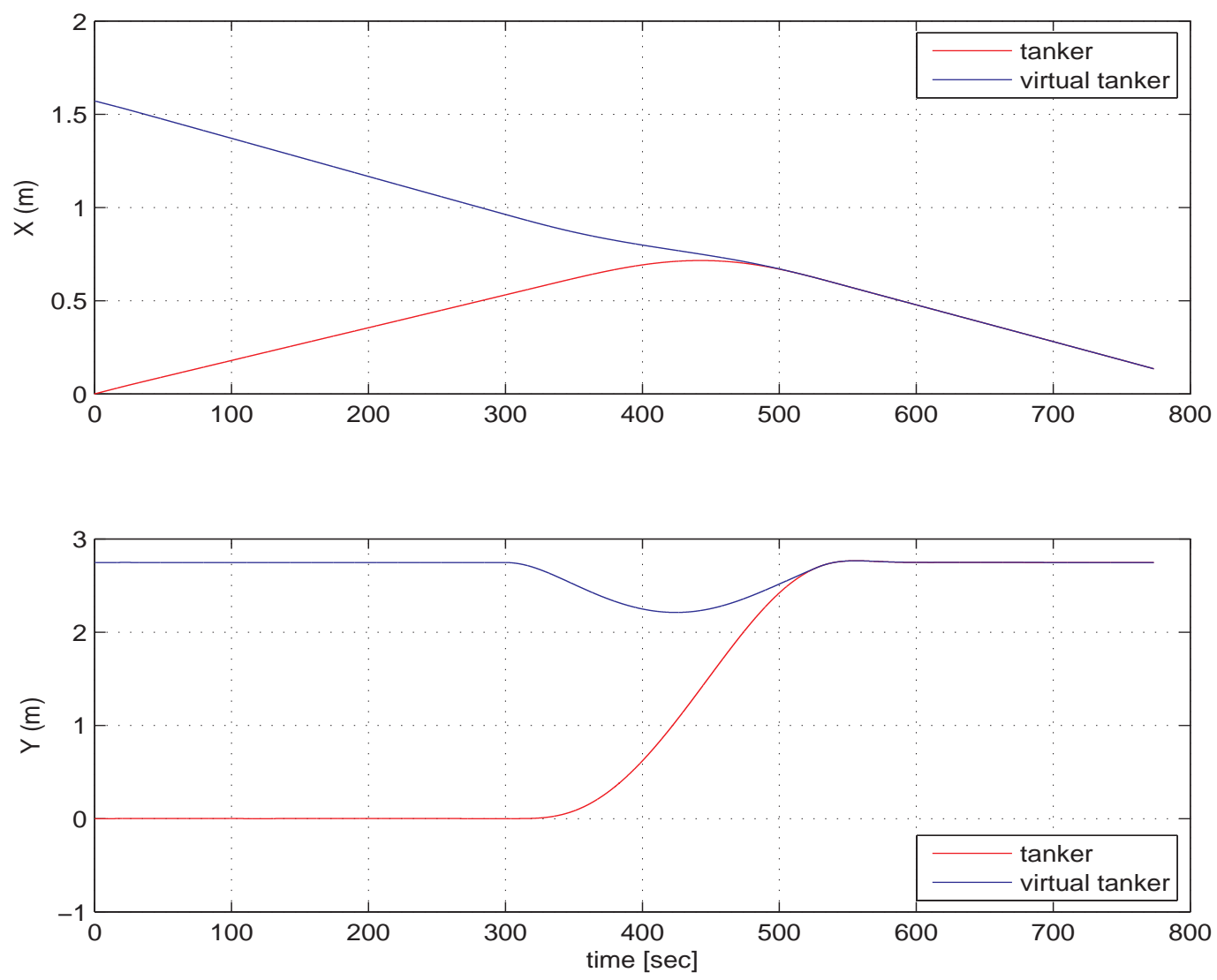

Figure 4.35. Tanker versus virtual tanker position trajectory in $\mathrm{x}-\mathrm{y}$ axis.

The tanker aircraft control efforts are shown in Fig. 4.40. The control surface deflections and the throttle setting are small throughout the whole refueling orbit despite the prevailing wind.

The receiver aircraft control efforts are shown in Fig. 4.41. All control surface deflections are small after the initial wind encounter until switching from the virtual tanker to the tanker aircraft. The throttle setting of the receiver aircraft has a transient that occurs at about 600 seconds. This is because of the kinematics level switching in the inertial velocity signal when the actual tanker meets the virtual tanker at the ARCP. However, the magnitude of transient is small relative to the nominal value. 

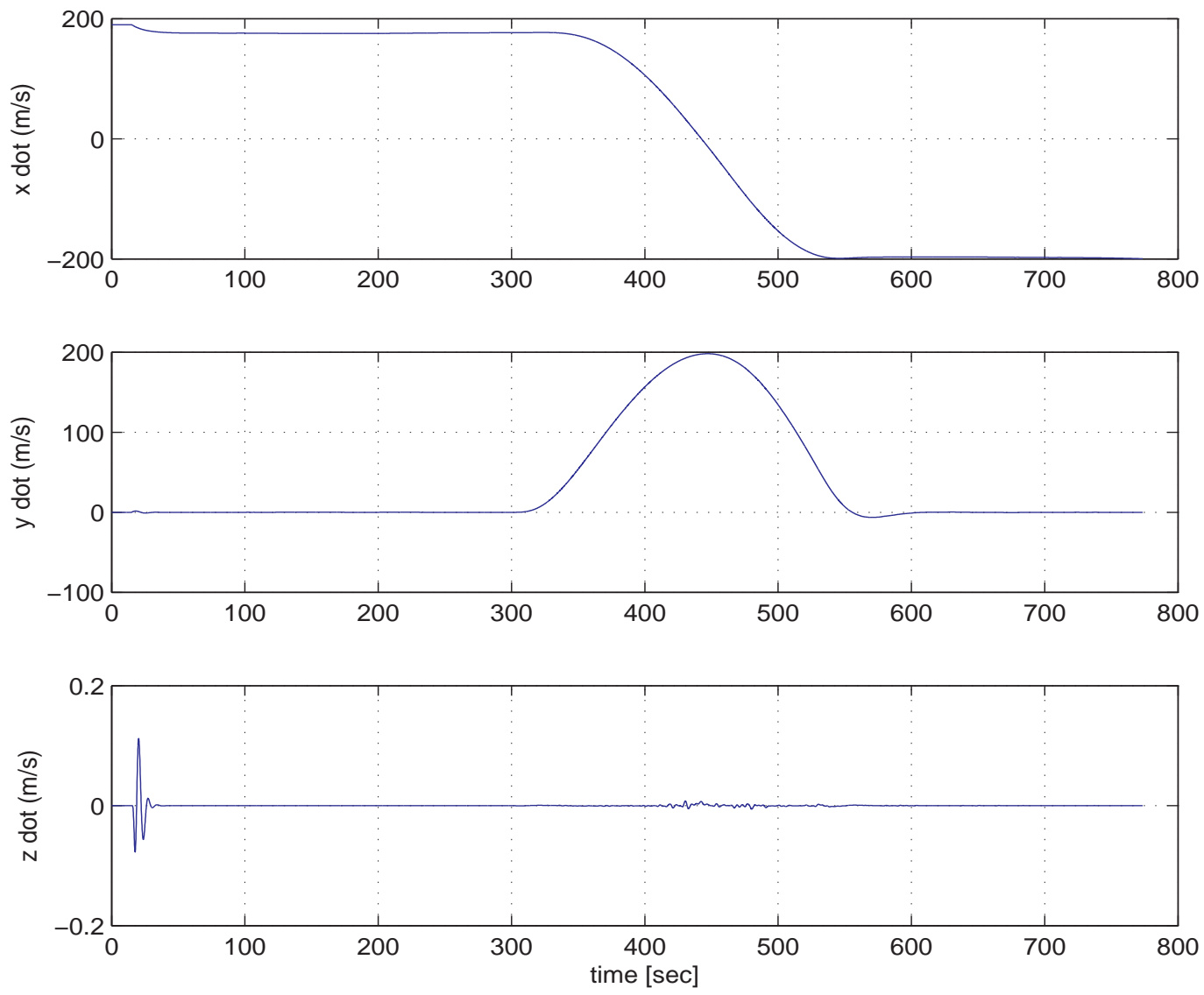

Figure 4.36. Tanker aircraft inertial velocities.

The receiver's aircraft relative position versus the commanded position in three axis of the tanker's body frame is shown in Fig. 4.42. The results show that when the prevailing wind is turned on, the $\mathrm{x}$ relative position maximum deviation is $38 \mathrm{~m}$, the $\mathrm{y}$ relative position maximum deviation is $11 \mathrm{~m}$ and the $\mathrm{z}$ relative position maximum deviation was $16 \mathrm{~m}$. Then the position controller is capable of handling the effect of the wind. Another set of transients is observed when switching from the virtual tanker to the tanker aircraft with. During this transients, the maximum deflection in x-relative position is $8 \mathrm{~m}$, the maximum deflection in y-relative position is $6 \mathrm{~m}$ in z-relative position is $4 \mathrm{~m}$. After switched to following the actual tanker, the receiver 

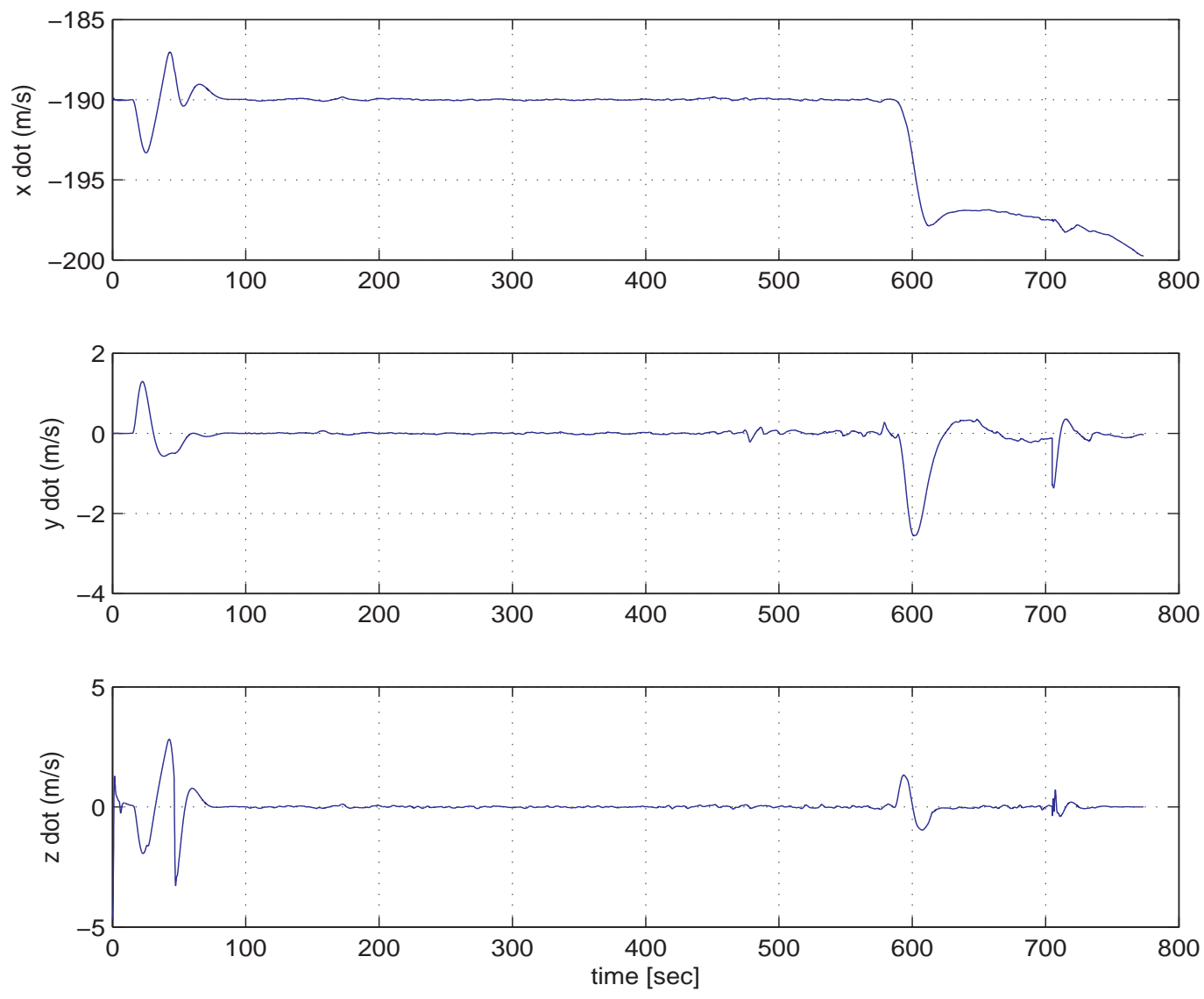

Figure 4.37. Receiver aircraft inertial velocities.

controller stabilizes the aircraft at the precontract position despite the prevailing wind. 

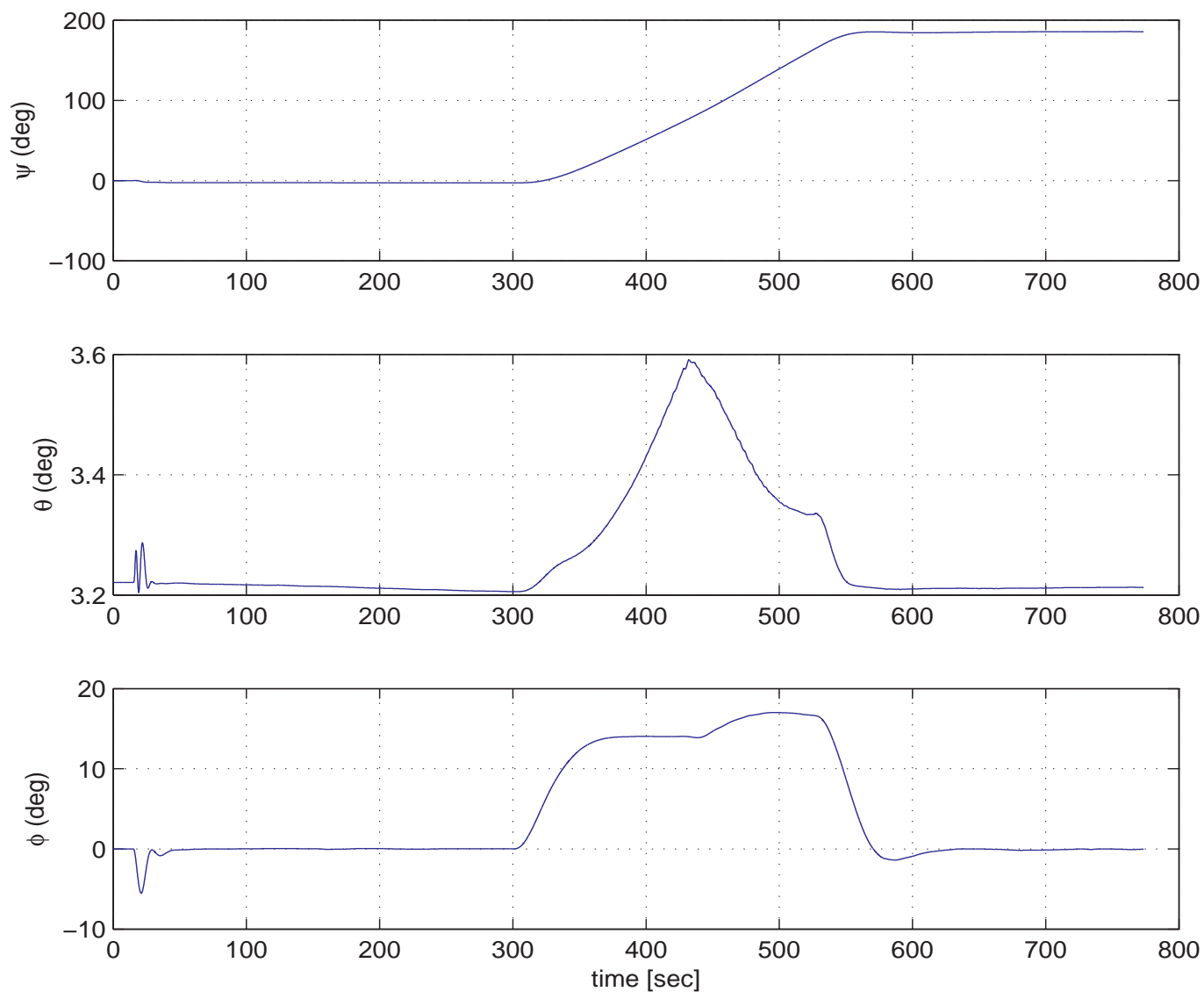

Figure 4.38. Tanker aircraft Euler's angle. 

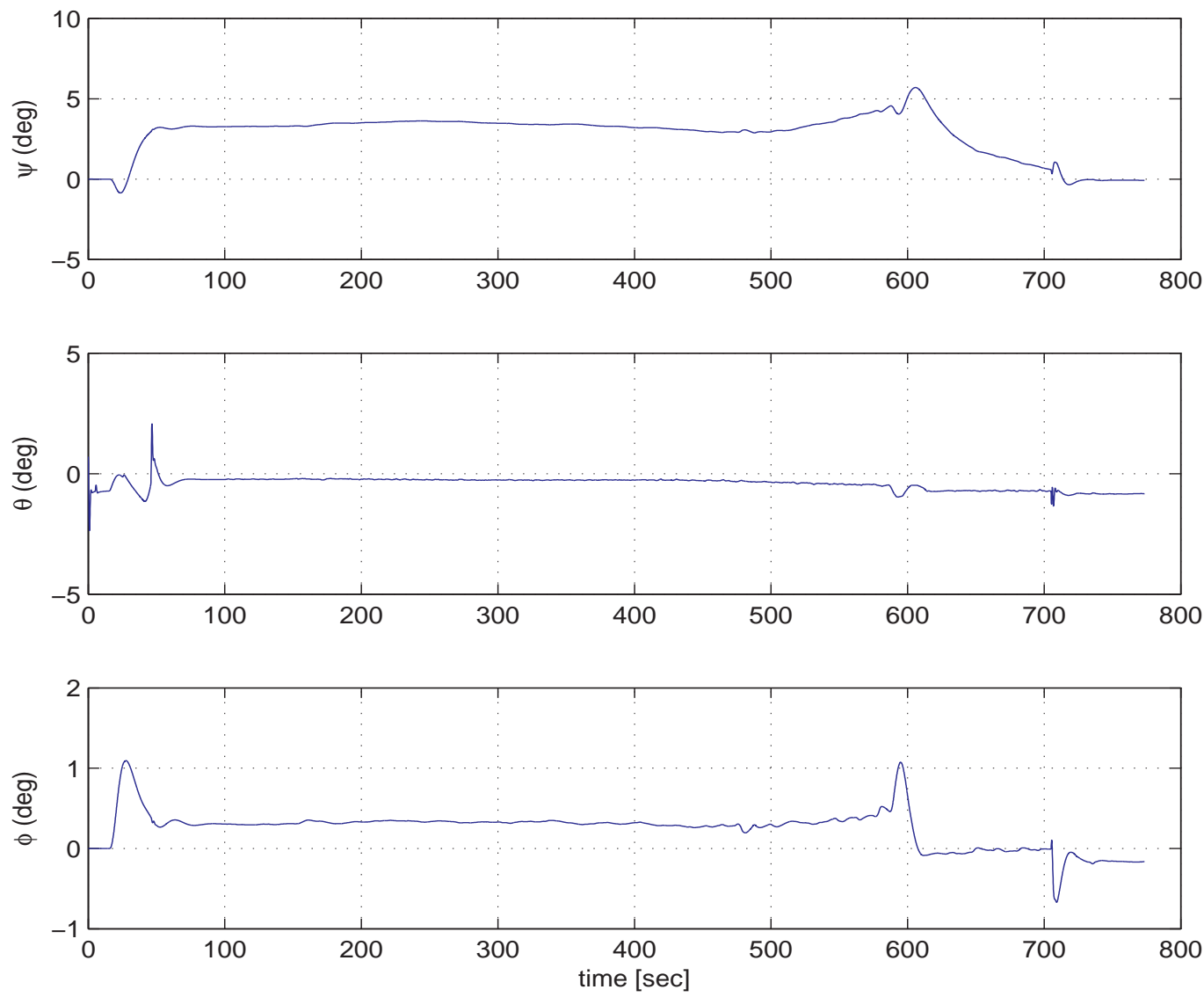

Figure 4.39. Receiver aircraft Euler's angle. 

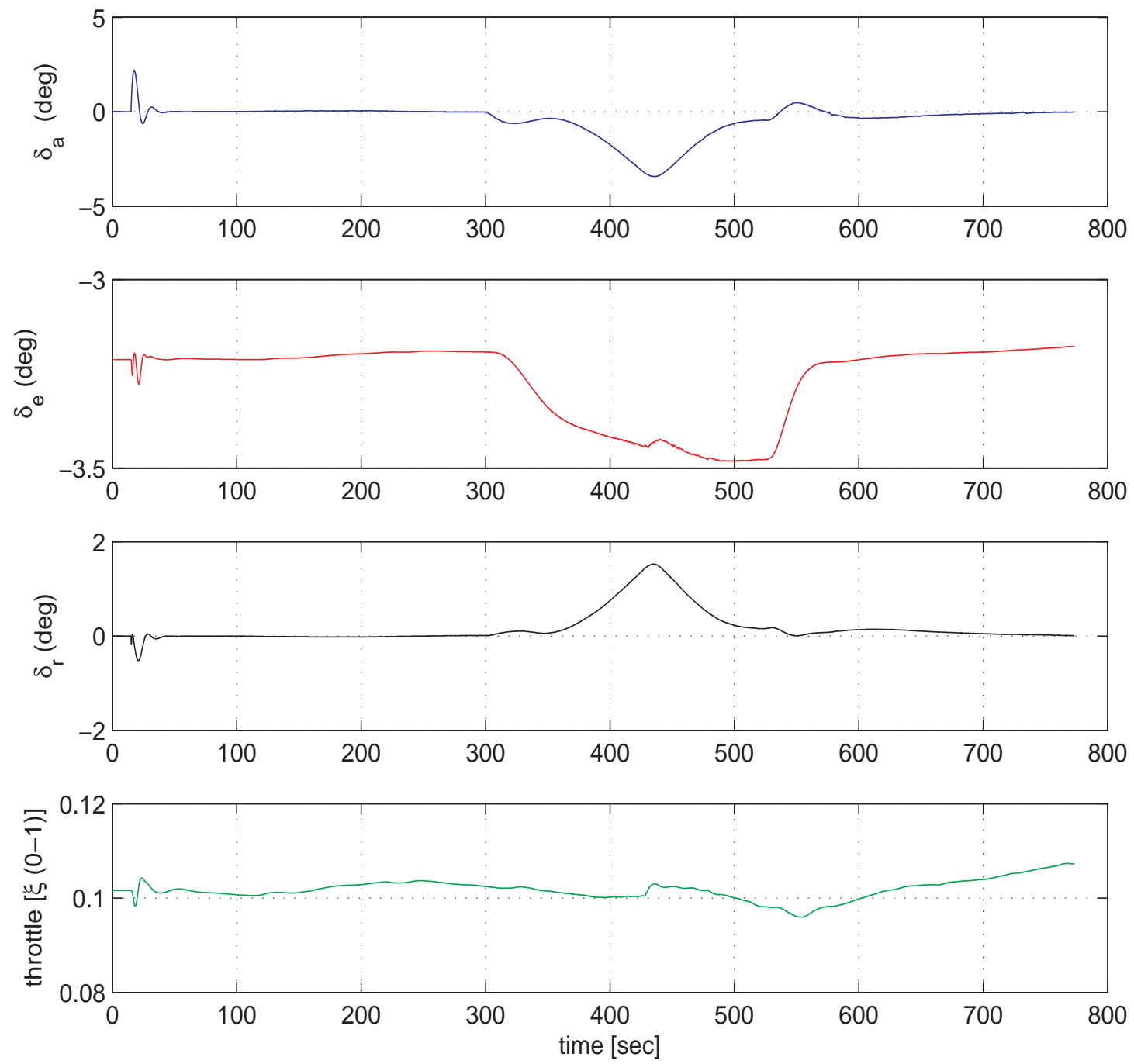

Figure 4.40. Tanker aircraft control signals. 

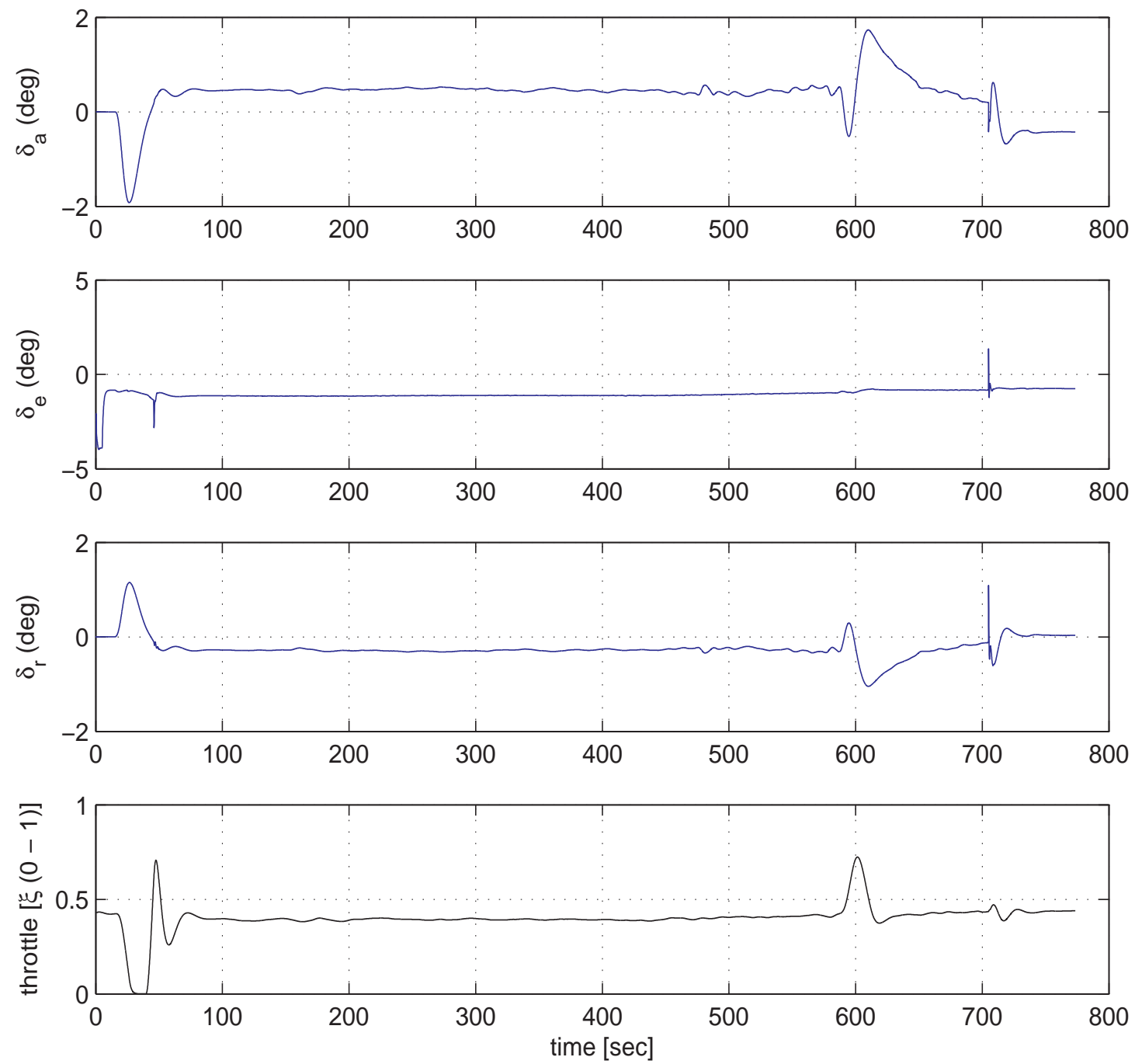

Figure 4.41. Receiver aircraft control signals. 

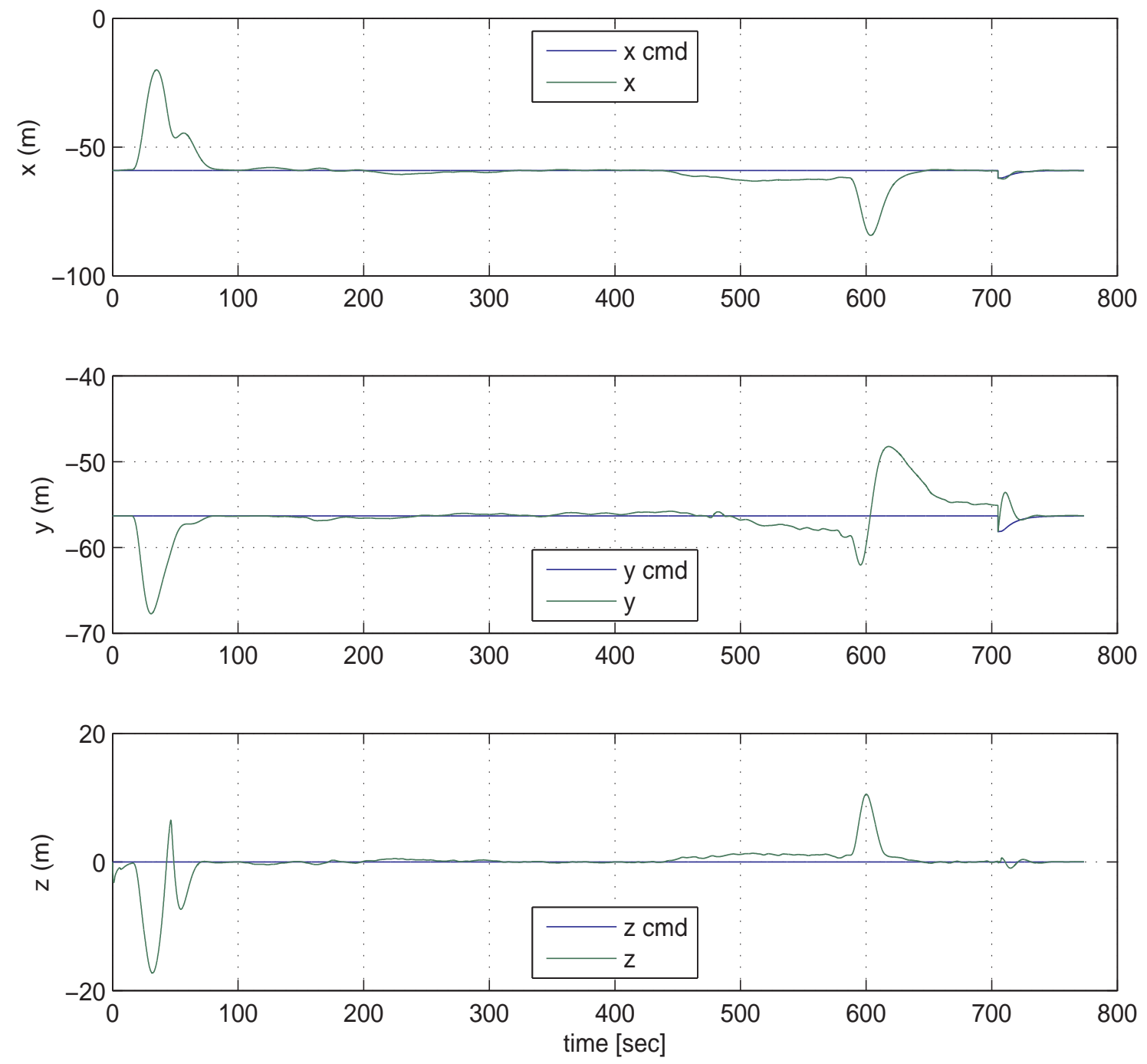

Figure 4.42. Receiver aircraft's relative position. 


\section{CHAPTER 5}

\section{CONCLUSION AND RECOMMENDATION FOR FUTURE WORK}

A guidance algorithm for the receiver aircraft to rendezvous with the tanker in the presence of prevailing wind, that can be applied to automated aerial refueling is developed in this thesis. Existing aerial refueling rendezvous procedures for piloted aircraft are investigated. The point parallel and enroute rendezvous procedures are determined to be applicable for UAV systems. A modified version of point-parallel rendezvous procedure is defined and a guidance algorithm for the receiver aircraft is developed. The modification is only responsible to fly along the aerial refueling orbit and it is the receiver's task to synchronize its motion with the tanker to rendezvous at a geographically fixed point along the aerial refueling line. This rendezvous procedure is more appropriate of there are multiple receiver approaching for rendezvous from different directions and the designated airspace for aerial refueling operation is limited.

For a realistic simulation of aerial refueling rendezvous, full 6-DOF aircraft models for both tanker and receiver are used in the simulations. To keep the focus of the thesis work on development of guidance algorithms, the existing low level controllers of both aircraft are used. This imposes an additional requirement of the guidance algorithms that they should be compatible with the controllers such that they generate commanded signals that can be processed by the controllers.

The existing controller of the tanker accepts commanded airspeed, altitude and yaw rate. A nonlinear guidance algorithm is utilized that minimized cross-track errors while tracking a moving point on a curved trajectory. A concept of virtual target is proven to be effective in generating a trajectory that can be followed by 
the nonlinear guidance algorithm to fly the tanker on the aerial refueling orbit. The simulation results show that the tanker can fly, with minimal deviations, along the refueling orbit in the absence of prevailing wind. Only during the start and end of a turn there are some small deviations. However, in the presence of prevailing wind, there are noticeable deviations from the refueling orbit, especially during the turns. One weakness of the nonlinear guidance algorithm observed is the difficulty in keeping the longitudinal distance with the virtual target in case of tail and head wind. This is also partly due to the fact that the tanker controller controls the airspeed and the ground speed of the tanker. Since the tanker is subject to the effect of the prevailing wind while the virtual target follows perfectly an inertially fixed trajectory, a proportional controller is used for preventing the tanker to pass the virtual target in the case of tailwind or to fall far behind in the case of headwind. It is shown that the nonlinear guidance algorithm used to eliminate the cross-track error fails to generate feasible yaw rate commands when the tanker is ahead of the virtual target. Overall, the existing tanker controller to fly the tanker along the refueling orbit with small control efforts. The deviations from the refueling orbit or variation of speed due to the prevailing wind are tolerated to have a realistic tanker response to wind exposure. Such a realistic tanker response to wind exposure makes the simulation environment more realistic for the evaluation of the receiver rendezvous guidance and control system.

For the receiver aircraft to successfully rendezvous with the tanker, the concept of virtual tanker is introduced. This is motivated by the fact that the receiver low-lever controller is capable of moving the receiver along a commanded trajectory relative to a moving and rotating frame. To move the receiver along a trajectory defined within the inertial frame, it suffices to simply define the motion of the reference frame and command the receiver to hold a fixed position relative to this maneuvering 
reference frame. In the case of aerial refueling rendezvous, this maneuvering reference frame, called "virtual tanker" is synchronized with the motion of the actual tanker flying along the refueling orbit. The synchronization of the virtual tanker flying along the refueling line towards the rendezvous point to make it possible for the receiver to adjust its trajectory to ensure timely arrival at the rendezvous point. Further, commanding the receiver to fly at the "pre-contract" position of the virtual tanker facilitates the establishment of the formation with the actual tanker after the rendezvous.

There are various directions for extension of this work. While successful rendezvous by the receiver aircraft in the presence of prevailing wind has been demonstrated without direct collaboration between the receiver and tanker, possible collaboration schemes should be studied. In the current implementation, the tanker only tries to fly on predefined aerial refueling orbit while the full responsibility for successful rendezvous is on the receiver. Different collaboration schemes that split the responsibility between the receiver and the tanker can be investigated. Another direction of future work is to employ the guidance algorithms developed in this thesis to other rendezvous procedures such as enroute rendezvous. Development of multiple UAV rendezvous with the tanker aircraft is another subject of future work. This work has dealt with the rendezvous of a single receiver with the tanker. When multiple receivers are flying for aerial refueling, their collaboration with each other and the tanker should be investigated. Some ideas of direct extension of this work follow. In the simulation, different flight conditions should be tested for the evaluation of the robustness of the guidance algorithms. For example, the orientation of the refueling orbit relative to the inertial frame should be changed and the same simulation of rendezvous should be repeated. Different initial positions of the tanker and receiver should be tried. This work assume that the communication between the tanker and 
the UAV is continuous. The effects of communication of sampled signals at different rates and delay present in transferred data should be investigated. In addition to the prevailing wind, atmosphere turbulence should be turned on to study the sensitivity of the guidance algorithms. 
APPENDIX A

THE AERIAL REFUELING TERMINOLOGY 
There are various air refueling terminologies that are used in this thesis. The definitions are taken from [4].

Air refueling track $=\mathrm{A}$ track designated for air refueling reserved by the receiver unit/planner. If possible, the track from the ARIP to the ARCP should be along a TACAN/VORTAC radial and within $100 \mathrm{~nm}$ of the station.

Air Refueling Initial Point $(\mathrm{ARIP})=$ A point located upstream from the ARCP at which the receiver aircraft initiates a rendezvous with the tanker.

Air Refueling Control Point $(\mathrm{ARCP})=$ The planned geographic point over which the receiver(s) arrive in the observation/precontact position with respect to the assigned tanker.

Air Refueling Control Time $(\mathrm{ARCT})=$ The planned time that the receiver and tanker will arrive over the air refueling control point (ARCP).

Air Refueling Exit Point (AR EXIT PT) = The designated geographic point at which the refueling track terminates. In a refueling anchor, it is a designated point where the tanker and rceiver may depart the anchor area after refueling is completed.

Anchor point $=$ A designated geographical point on the downstream at the end of the inbound course of the Anchor Refueling Pattern.

Anchor refueling $=$ Air refueling performed as the tankers maintain a prescribed pattern which is anchored to a geographical point.

Anchor Rendezvous $=$ The procedures normally employed by radar (CRC/ GCI/ AWACS) to vector the tanker(s) and receiver(s) for a visual join-up for refueling. 
APPENDIX B

SCALAR EQUATIONS OF MOTION OF THE TANKER 
A scalar equations of motion of the tanker aircraft are shown in the following equations.

Translational Kinematics:

$$
\begin{gathered}
\dot{x}_{T}=V_{T}\left[\cos \beta_{T} \cos \alpha_{T} \cos \theta_{T} \cos \psi_{T}+\sin \beta_{T}\left(-\cos \phi_{T} \sin \psi_{T}+\sin \phi_{T} \sin \theta_{T} \cos \psi_{T}\right)\right. \\
\left.+\cos \beta_{T} \sin \alpha_{T}\left(\sin \phi_{T} \sin \psi_{T}+\cos \phi_{T} \sin \theta_{T} \cos \psi_{T}\right)\right] \\
\dot{y}_{T}=V_{T}\left[\cos \beta_{T} \cos \alpha_{T} \cos \theta_{T} \sin \psi_{T}+\sin \beta_{T}\left(\cos \phi_{T} \cos \psi_{T}+\sin \phi_{T} \sin \theta_{T} \cos \psi_{T}\right)\right. \\
\left.+\cos \beta_{T} \sin \alpha_{T}\left(-\sin \phi_{T} \cos \psi_{T}+\cos \phi_{T} \sin \theta_{T} \sin \psi_{T}\right)\right] \\
\dot{z}_{T}=V_{T}\left[-\cos \beta_{T} \cos \alpha_{T} \sin \theta_{T}+\sin \beta_{T} \sin \phi_{T} \cos \theta_{T}\right. \\
\left.+\cos \beta_{T} \sin \alpha_{T} \cos \phi_{T} \cos \theta_{T}\right]
\end{gathered}
$$

where $\left(x_{T}, y_{T}, z_{T}\right)$ is the position of the tanker aircraft relative the inertial frame, $\left(\psi_{T}, \theta_{T}, \phi_{T}\right)$ is the orientation of the tanker relative to the inertial frame in terms of the Euler angles, $\left(V_{T}, \beta_{T}, \alpha_{T}\right)$ are the airspeed, side slip angle and angle-of-attack of the tanker. Translational Dynamics:

$$
\begin{aligned}
\dot{V}_{T}= & g\left[\cos \theta_{T} \sin \beta_{T} \sin \phi_{T}+\cos \beta_{T}\left(\cos \phi_{T} \cos \theta_{T} \sin \alpha_{T}-\cos \alpha_{T} \sin \theta_{T}\right)\right] \\
& +\frac{1}{m_{T}}\left[-D_{T}+T_{T} \cos \left(\alpha_{T}+\delta_{T}\right) \cos \beta_{T}\right] \\
\dot{\beta}_{T}= & -r_{T} \cos \alpha_{T}+p_{T} \sin \alpha_{T} \\
& +\frac{g}{V_{T}}\left[-\cos \phi_{T} \cos \theta_{T} \sin \alpha_{T} \sin \beta_{T}+\cos \beta_{T} \cos \theta_{T} \sin \phi_{T}+\cos \alpha_{T} \sin \beta_{T} \sin \theta_{T}\right] \\
& -\frac{1}{m_{T} V_{T}}\left[S_{T}+T_{T} \cos \left(\alpha_{T}+\delta_{T}\right) \sin \beta_{T}\right] \\
\dot{\alpha}_{T}= & q_{T}-\left(p_{T} \cos \alpha_{T}+r_{T} \sin \alpha_{T}\right) \tan \beta_{T} \\
& +\frac{g \sec \beta_{T}}{V_{T}}\left[\cos \alpha_{T} \cos \phi_{T} \cos \theta_{T}+\sin \alpha_{T} \sin \theta_{T}\right] \\
& -\frac{\sec \beta_{T}}{m_{T} V_{T}}\left[L_{T}+T_{T} \sin \left(\alpha_{T}+\delta_{T}\right)\right]
\end{aligned}
$$


The external forces acting on the tanker are the gravitational force $M_{T}$ (expressed in the inertial frame), the aerodynamic force $A_{T}$ (expressed in the wind frame of the tanker) and propulsive force $P_{T}$ (expressed in the body frame of the tanker). In general, the representations of the forces are

$$
M_{T}=\left[\begin{array}{c}
0 \\
0 \\
m_{T} g
\end{array}\right] \quad A_{T}=\left[\begin{array}{c}
-D_{T} \\
-S_{T} \\
-L_{T}
\end{array}\right] \quad P_{T}=\left[\begin{array}{c}
T_{T} \cos \delta_{T} \\
0 \\
-T_{T} \sin \delta_{T}
\end{array}\right]
$$

where $g$ is the gravitational acceleration, $m_{T}$ is the mass of the tanker, $\left(D_{T}, S_{T}, L_{T}\right)$ are the drag, side force and lift on the tanker, respectively, $T_{T}$ is the thrust magnitude, and $\delta_{T}$ is the thrust inclination angle. Also, note that $\mathbf{S}(\cdot)$ is the skew-symmetric matrix operation on the representation of a vector and defined as

$$
\mathbf{S}(x)=\left[\begin{array}{ccc}
0 & x_{3} & -x_{2} \\
-x_{3} & 0 & x_{1} \\
x_{2} & -x_{1} & 0
\end{array}\right]
$$

for an arbitrary vector $\underline{x}$ with the representation $\left[\begin{array}{lll}x_{1} & x_{2} & x_{3}\end{array}\right]^{T}$. where $\left(p_{T}, q_{T}, r_{T}\right)$ is the angular velocity of the tanker expressed in the tanker's body frame. The aerodynamic forces are given by the following standard expressions

$$
\begin{aligned}
D_{T} & =\frac{1}{2} \rho V_{T}^{2} \mathcal{S}_{T} C_{D_{T}}, \\
S_{T} & =\frac{1}{2} \rho V_{T}^{2} \mathcal{S}_{T} C_{S_{T}}, \\
L_{T} & =\frac{1}{2} \rho V_{T}^{2} \mathcal{S}_{T} C_{L_{T}},
\end{aligned}
$$


where $\mathcal{S}_{T}$ is the reference area of the tanker and $\rho$ is the ambient air density. The aerodynamic coefficients are

$$
\begin{aligned}
C_{D_{T}} & =C_{D 0}+C_{D \alpha^{2}} \alpha_{T}^{2} \\
C_{S_{T}} & =C_{S 0}+C_{S \beta} \beta_{T}+C_{S \delta_{r}} \delta_{r_{T}} \\
C_{L_{\text {wing }}} & =C_{L 0}+C_{L \alpha} \alpha_{T}+C_{L \alpha 2}\left(\alpha_{T}-\alpha_{r e f}\right)^{2}+C_{L q} \frac{c_{T}}{2 V_{T}} q_{T} \\
C_{L_{\text {tail }}} & =C_{L \delta_{e}} \delta_{e_{T}} \\
C_{L_{T}} & =C_{L_{\text {wing }}}+C_{L_{\text {tail }}}
\end{aligned}
$$

where $\left(\delta_{a_{T}}, \delta_{e_{T}}, \delta_{r_{T}}\right)$ are the deflections of the control surfaces (aileron, elevator, rudder, respectively) and $c_{T}$ is the chord length for the tanker. Rotational Kinematics:

$$
\begin{aligned}
\dot{\phi}_{T} & =p_{T}+q_{T} \sin \phi_{T} \tan \theta_{T}+r \cos \phi_{T} \tan \theta_{T} \\
\dot{\theta}_{T} & =q_{T} \cos \phi_{T}-r \sin \phi_{T} \\
\dot{\psi}_{T} & =\left(q_{T} \sin \phi_{T}+r_{T} \cos \phi_{T}\right) \sec \theta_{T}
\end{aligned}
$$

where note that both the orientation in terms of $\left(\psi_{T}, \theta_{T}, \phi_{T}\right)$, and the angular velocity, $\left(p_{T}, q_{T}, r_{T}\right)$, of the tanker are relative to the inertial frame. Rotational Dynamics:

$$
\begin{aligned}
& \dot{p}_{T}=\frac{1}{\left(I_{x x} I_{z z}-I_{x z}^{2}\right)}[\left(I_{x x}-I_{y y}+I_{z z}\right) I_{x z} p_{T} q_{T}+\left(I_{y y}-I_{z z}+I_{z z}^{2}-I_{x z}^{2}\right) q_{T} r_{T} \\
&\left.+I_{z z} \mathcal{L}_{T}+I_{x z} \mathcal{N}_{T}\right] \\
& \dot{q}_{T}=\frac{1}{I_{y y}}\left[\left(I_{z z}-I_{x x}\right) p_{T} r_{T}+\left(r_{T}^{2}-p_{T}^{2}\right) I_{x z}+\mathcal{M}_{T}\right] \\
& \dot{r}_{T}=\frac{1}{\left(I_{x x} I_{z z}-I_{x z}^{2}\right)}\left[\left(I_{x x}^{2}-I_{x x} I_{y y}+I_{x z}^{2}\right) p_{T} q_{T}+\left(-I_{x x}+I_{y y}-I_{z z}\right) I_{x z} q_{T} r_{T}\right. \\
&\left.\quad+I_{x z} \mathcal{L}_{T}+I_{x x} \mathcal{N}_{T}\right]
\end{aligned}
$$

where $I_{(\cdot)(\cdot)}$ is the moment or product of inertia of the tanker relative to the corresponding axis of the tanker's body frame. Note here also that the notation for $I_{(\cdot)(\cdot)}$ 
is the same for both tanker and the receiver while their values are obviously different. $\left(\mathcal{L}_{T}, \mathcal{M}_{T}, \mathcal{N}_{T}\right)$ are the rolling, pitching and yawing moments, respectively.

$$
\begin{aligned}
\mathcal{L}_{T} & =\frac{1}{2} \rho V_{T}^{2} \mathcal{S}_{T} b_{T} C_{\mathcal{L}_{T}} \\
\mathcal{M}_{T} & =\frac{1}{2} \rho V_{T}^{2} \mathcal{S}_{T} c_{T} C_{\mathcal{M}_{T}}+\Delta_{z_{T}} T_{T} \\
\mathcal{N}_{T} & =\frac{1}{2} \rho V_{T}^{2} \mathcal{S}_{T} b_{T} C_{\mathcal{N}_{T}}
\end{aligned}
$$

where $b_{T}$ is the wingspan of the tanker aircraft and $\Delta_{z_{T}}$ is the moment arms of the thrust in the tanker's body frame. The aerodynamic moment coefficients are

$$
\begin{aligned}
C_{\mathcal{L}_{T}} & =C_{\mathcal{L} 0}+C_{\mathcal{L} \delta_{a}} \delta_{a_{T}}+C_{\mathcal{L} \delta_{r}} \delta_{r_{T}}+C_{\mathcal{L} \beta} \beta_{T}+C_{\mathcal{L} p} \frac{b_{T}}{2 V_{T}} p_{T}+C_{\mathcal{L} r} \frac{b_{T}}{2 V_{T}} r_{T} \\
C_{\mathcal{M}_{T}} & =C_{\mathcal{L} \alpha} \alpha_{T}+C_{\mathcal{L} \delta_{e}} \delta_{e_{T}}+C_{\mathcal{M} q} \frac{c_{T}}{2 V_{T}} q_{T} \\
C_{\mathcal{N}_{T}} & =C_{\mathcal{N} 0}+C_{\mathcal{N} \delta_{a}} \delta_{a_{T}}+C_{\mathcal{N} \delta_{r}} \delta_{r_{T}}+C_{\mathcal{N} \beta} \beta_{T}+C_{\mathcal{N} p} \frac{b_{T}}{2 V_{T}} p_{T}+C_{\mathcal{N} r} \frac{b_{T}}{2 V_{T}} r_{T}
\end{aligned}
$$


APPENDIX C

SCALAR EQUATIONS OF MOTION OF THE RECEIVER 
A scalar equations of motions of the receiver aircraft are shown in the following equations.

Translational Kinematics:

$$
\begin{aligned}
\dot{x}=V & {[\cos \beta \cos \alpha \cos \theta \cos \psi+\sin \beta(-\cos \phi \sin \psi+\sin \phi \sin \theta \cos \psi)} \\
& +\cos \beta \sin \alpha(\sin \phi \sin \psi+\cos \phi \sin \theta \cos \psi)] \\
& -\cos \theta_{T} \cos \psi_{T} V_{x T}-\cos \theta_{T} \sin \psi_{T} V_{y T}+\sin \theta_{T} V_{z T} \\
& +r_{T} y-q_{T} z \\
\dot{y}=V & {[\cos \beta \cos \alpha \cos \theta \sin \psi+\sin \beta(\cos \phi \cos \psi+\sin \phi \sin \theta \cos \psi)} \\
& +\cos \beta \sin \alpha(-\sin \phi \cos \psi+\cos \phi \sin \theta \sin \psi)] \\
- & \left(-\cos \phi_{T} \sin \psi_{T}+\sin \phi_{T} \sin \theta_{T} \cos \psi_{T}\right) V_{x T} \\
- & \left(\cos \phi_{T} \cos \psi_{T}+\sin \phi_{T} \sin \theta_{T} \sin \psi_{T}\right) V_{y T}+\sin \phi_{T} \cos \theta_{T} V_{z T} \\
& -r_{T} x+p_{T} z \\
\dot{z}=V & {[-\cos \beta \cos \alpha \sin \theta+\sin \beta \sin \phi \cos \theta+\cos \beta \sin \alpha \cos \phi \cos \theta] } \\
- & \left(\sin \phi_{T} \sin \psi_{T}+\cos \phi_{T} \sin \theta_{T} \cos \psi_{T}\right) V_{x T} \\
- & \left(-\sin \phi_{T} \cos \psi_{T}+\cos \phi_{T} \sin \theta_{T} \sin \psi_{T}\right) V_{y T}-\cos \phi_{T} \cos \theta_{T} V_{z T} \\
+ & q_{T} x-p_{T} y \\
& \\
&
\end{aligned}
$$

where $(x, y, z)$ is the position of the receiver aircraft relative to the tanker, $(\psi, \theta, \phi)$ is the orientation of the receiver relative to the tanker in terms of the Euler angles, $(V, \beta, \alpha)$ are the airspeed, side slip angle and angle-of-attack of the receiver, $\left(\psi_{T}, \theta_{T}, \phi_{T}\right)$ is the orientation of the tanker relative to the inertial frame. Recall that $\left(p_{T}, q_{T}, r_{T}\right)$ are the components of the angular velocity of the tanker relative to the inertial frame, and $\left(V_{x T}, V_{y T}, V_{z T}\right)$ are the components of the velocity of the tanker 
relative to the inertial frame. Note that $V_{x T}=\dot{x}_{T}$ in Eq. (B.1), $V_{y T}=\dot{y}_{T}$ in Eq. (B.2), and $V_{z T}=\dot{z}_{T}$ in Eq. (B.3). Translational Dynamics:

$$
\begin{aligned}
& \dot{V}=g\left\{\cos \alpha \cos \beta\left(-\cos \theta \cos \psi \cos \theta_{T}+\cos \theta \sin \psi \sin \phi_{T} \cos \theta_{T}-\sin \theta \cos \phi_{T} \cos \theta_{T}\right)\right. \\
& +\sin \beta\left[-(-\cos \phi \sin \psi+\sin \phi \sin \theta \cos \psi) \sin \theta_{T}\right. \\
& +(\cos \phi \cos \psi+\sin \phi \sin \theta \sin \psi) \sin \phi_{T} \cos \theta_{T} \\
& \left.+\sin \phi \cos \theta \cos \phi_{T} \cos \theta_{T}\right] \\
& +\cos \beta \sin \alpha\left[-(\sin \phi \sin \psi+\cos \phi \sin \theta \cos \psi) \sin \theta_{T}\right. \\
& +(-\sin \phi \cos \psi+\cos \phi \sin \theta \sin \psi) \sin \phi_{T} \cos \theta_{T} \\
& \left.\left.+\cos \phi \cos \theta \cos \phi_{T} \cos \theta_{T}\right]\right\} \\
& +\frac{1}{m_{R}}\left(-D+T_{x} \cos \alpha \cos \beta+T_{y} \sin \beta+T_{z} \cos \beta \sin \alpha\right) \\
& \dot{\beta}=\sin \alpha\left(p+p_{T} \cos \psi \cos \theta+q_{T} \sin \psi \cos \theta-r_{T} \sin \theta\right) \\
& -\cos \alpha\left[r+p_{T}(\sin \phi \sin \psi+\cos \phi \cos \psi \sin \theta)+q_{T}(\sin \theta \cos \phi \sin \psi-\sin \phi \cos \psi)\right. \\
& \left.+r_{T} \cos \phi \cos \theta\right] \\
& +\frac{g}{V}\left\{-\cos \alpha \sin \beta\left(-\cos \theta \cos \psi \sin \theta_{T}+\cos \theta \sin \psi \sin \phi_{T} \cos \theta_{T}-\sin \theta \cos \phi_{T} \cos \theta_{T}\right)\right. \\
& +\cos \beta\left[-(-\cos \phi \sin \psi+\sin \phi \sin \theta \cos \psi) \sin \theta_{T}\right. \\
& \left.+(\cos \phi \cos \psi+\sin \phi \sin \theta \sin \psi) \sin \phi_{T} \cos \theta_{T}+\sin \phi \cos \theta \cos \phi_{T} \cos \theta_{T}\right] \\
& -\sin \alpha \sin \beta\left[-(\sin \phi \sin \psi+\cos \phi \sin \theta \cos \psi) \sin \theta_{T}\right. \\
& +(-\sin \phi \cos \psi+\cos \phi \sin \theta \sin \psi) \sin \phi_{T} \cos \theta_{T} \\
& \left.\left.+\cos \phi \cos \theta \cos \phi_{T} \cos \theta_{T}\right]\right\} \\
& +\frac{1}{m_{R} V}\left(-S-T_{x} \cos \alpha \sin \beta+T_{y} \cos \beta-T_{z} \sin \alpha \sin \beta\right)
\end{aligned}
$$




$$
\begin{aligned}
& \dot{\alpha}=q-p_{T}(\cos \phi \sin \psi-\sin \theta \sin \phi \cos \psi)+q_{T}(\cos \phi \cos \psi+\sin \theta \sin \phi \sin \psi) \\
& +r_{T} \sin \phi \cos \theta \\
& -\sin \alpha \tan \beta\left[r+p_{T}(\sin \psi \sin \phi+\cos \psi \sin \theta \cos \phi)+q_{T}(\sin \psi \sin \theta \cos \phi-\sin \psi \sin \phi)\right. \\
& \left.\quad+r_{T} \cos \phi \cos \theta\right] \\
& +\cos \alpha \tan \beta\left(-p-p_{T} \cos \psi \cos \theta-q_{T} \sin \psi \cos \theta+r_{T} \sin \theta\right) \\
& +\frac{g}{V}\left\{-\sec \beta \sin \alpha\left(-\cos \theta \cos \psi \sin \theta_{T}+\cos \theta \sin \psi \sin \phi_{T} \cos \theta_{T}-\sin \theta \cos \phi_{T} \cos \theta_{T}\right)\right. \\
& \quad+\cos \alpha \sec \beta\left[-(\sin \phi \sin \psi+\cos \phi \sin \theta \cos \psi) \sin \theta_{T}\right. \\
& \quad+(-\sin \phi \cos \psi+\cos \phi \sin \theta \sin \psi) \sin \phi_{T} \cos \theta_{T} \\
& \left.\left.\quad+\cos \phi \cos \theta \cos \phi_{T} \cos \theta_{T}\right]\right\} \\
& +\frac{\sec \beta}{m_{R} V}\left(-L-T_{x} \sin \alpha+T_{z} \cos \alpha\right)
\end{aligned}
$$

Note that the motion of the tanker aircraft -both translational and rotational- is represented as exogenous inputs in the translational equations of motion of the receiver aircraft. The variables included in this category are translational velocity $\left(V_{x T}, V_{y T}, V_{z T}\right)$, orientation in terms of Euler angles $\left(\psi_{T}, \theta_{T}, \phi_{T}\right)$ and angular velocity $\left(p_{T}, q_{T}, r_{T}\right)$, all relative to the inertial frame.

The external forces acting on the receiver are the gravitational force $M_{R}$ (expressed in the inertial frame), the aerodynamic force $A_{R}$ (expressed in the wind frame of the receiver) and the propulsive force $P_{R}$ (expressed in the body frame of the receiver). In general, the representations of the forces $M_{R}, A_{R}$ and $P_{R}$ are

$$
M_{R}=\left[\begin{array}{c}
0 \\
0 \\
m_{R} g
\end{array}\right] \quad A_{R}=\left[\begin{array}{c}
-D \\
-S \\
-L
\end{array}\right] \quad P_{R}=\left[\begin{array}{c}
T_{x} \\
T_{y} \\
T_{z}
\end{array}\right]
$$


where $m_{R}$ is the mass of the receiver, $(D, S, L)$ are the drag, side force and lift on the receiver, respectively, and $\left(T_{x}, T_{y}, T_{z}\right)$ are the components of the thrust vector in the body frame of the receiver.

The propulsion force $P_{R}$ has three components, which are functions of thrust magnitude $T_{R}$ and the direction of the thrust vector. The thrust vectoring is parameterized by the angles of the thrust vector with the receiver's body $\mathrm{xy}^{-}$- and $\mathrm{xz}^{-}$- planes. The components of the thrust are

$$
\begin{aligned}
& T_{x}=T_{R} \cos \delta_{z} \cos \delta_{y} \\
& T_{y}=T_{R} \sin \delta_{z} \\
& T_{z}=T_{R} \cos \delta_{z} \sin \delta_{y}
\end{aligned}
$$

Note that a positive $\delta_{y}$ rotation of the thrust generates a positive thrust component in the positive $z$-direction while inducing a positive pitching moment (moment around y-axis). Similarly, a positive $\delta_{z}$ rotation of the thrust generates a positive thrust component in the positive $y$-direction while inducing a negative yawing moment (moment around z-axis).

The aerodynamic forces are given by

$$
\begin{aligned}
D & =\frac{1}{2} \rho V_{R}^{2} \mathcal{S}_{R} C_{D}, \\
S & =\frac{1}{2} \rho V_{R}^{2} \mathcal{S}_{R} C_{S}, \\
L & =\frac{1}{2} \rho V_{R}^{2} \mathcal{S}_{R} C_{L},
\end{aligned}
$$

where $\mathcal{S}_{R}$ is the reference area of the receiver. The aerodynamic coefficients are

$$
\begin{aligned}
C_{D} & =C_{D 0}+C_{D \alpha} \alpha+C_{D \alpha 2} \alpha^{2}+C_{D \delta_{e}} \delta_{e}+C_{D \delta_{e} 2} \delta_{e}^{2} \\
C_{S} & =C_{S 0}+C_{S \beta} \beta+C_{S \delta_{a}} \delta_{a}+C_{S \delta_{r}} \delta_{r} \\
C_{L} & =C_{L 0}+C_{L \alpha} \alpha+C_{L \alpha 2}\left(\alpha-\alpha_{r e f}\right)^{2}+C_{L q} \frac{c}{2 V_{R}} q_{r e l}+C_{L \delta_{e}} \delta_{e}
\end{aligned}
$$


where $\left(\delta_{a}, \delta_{e}, \delta_{r}\right)$ are the deflections of the control effectors (aileron, elevator, rudder) as the conventional control surfaces or (elevon, pitch flap, clamshell) as in the ICE (Innovative Control Effectors) aircraft, respectively. Note that, in Eq. (C.14), $q_{r e l}$ is the angular velocity of the receiver relative to the surrounding air around the body-y axis. However, in control design, $q_{r e l}=q$ since the wind is not considered. Rotational Kinematics:

$$
\begin{aligned}
\dot{\phi} & =p+q \sin \phi \tan \theta+r \cos \phi \tan \theta \\
\dot{\theta} & =q \cos \phi-r \sin \phi \\
\dot{\psi} & =(q \sin \phi+r \cos \phi) \sec \theta
\end{aligned}
$$

where note that both the orientation, $(\psi, \theta, \phi)$, and the angular velocity, $(p, q, r)$, of the receiver are relative to the tanker. Rotational Dynamics:

$$
\begin{aligned}
& \dot{p}=\frac{\left(I_{z z} \mathcal{L}+I_{x z} \mathcal{N}\right)}{\left(I_{x x} I_{z z}-I_{x z}^{2}\right)} \\
& +\frac{\left(I_{y y} I_{x x}-I_{z z}^{2}-I_{x z}^{2}\right)}{\left(I_{x x} I_{z z}-I_{x z}^{2}\right)}\left[q+p_{T}(\cos \psi \sin \theta \sin \phi-\sin \psi \cos \phi)\right. \\
& \left.+q_{T}(\sin \psi \sin \theta \sin \phi+\cos \psi \cos \phi)+r_{T} \cos \theta \sin \phi\right] \\
& {\left[r+p_{T}(\cos \psi \sin \theta \cos \phi+\sin \psi \sin \phi)\right.} \\
& \left.+q_{T}(\sin \psi \sin \theta \cos \phi-\cos \psi \sin \phi)+r_{T} \cos \theta \cos \phi\right] \\
& +\frac{\left(I_{y y} I_{x z}-I_{x x} I_{x z}-I_{z z} I_{x z}\right)}{\left(I_{x x} I_{z z}-I_{x z}^{2}\right)}\left[p+p_{T} \cos \psi \cos \theta+q_{T} \sin \psi \cos \theta-r_{T} \sin \theta\right] \\
& {\left[-q+p_{T}(\sin \psi \cos \phi-\cos \psi \sin \theta \sin \phi)\right.} \\
& \left.-q_{T}(\cos \psi \cos \phi-\sin \psi \sin \theta \sin \phi)-r_{T} \cos \theta \sin \phi\right] \\
& -r\left[p_{T}(\cos \psi \sin \theta \sin \phi-\sin \psi \cos \phi)+q_{T}(\sin \psi \sin \theta \sin \phi+\cos \psi \cos \phi)+r_{T} \cos \theta \sin \phi\right] \\
& +q\left[p_{T}(\cos \psi \sin \theta \cos \phi+\sin \psi \sin \phi)+q_{T}(\sin \psi \sin \theta \cos \phi-\cos \psi \sin \phi)\right. \\
& \left.+r_{T} \cos \theta \cos \phi\right]-\dot{p}_{T} \cos \psi \cos \theta-\dot{q}_{T} \sin \psi \cos \theta+\dot{r}_{T} \sin \theta
\end{aligned}
$$




$$
\begin{aligned}
& \dot{q}=\frac{\mathcal{M}}{I_{y y}}-\frac{I_{x z}}{I_{y y}}\left[p+p_{T} \cos \psi \cos \theta+q_{T} \sin \psi \cos \theta-r_{T} \sin \theta\right]^{2} \\
& +\frac{I_{x z}}{I_{y y}}\left[r+p_{T}(\cos \psi \sin \theta \cos \phi+\sin \psi \sin \phi)\right. \\
& \left.+q_{T}(\sin \psi \sin \theta \cos \phi-\cos \psi \sin \phi)+r_{T} \cos \theta \cos \phi\right]^{2} \\
& +\frac{\left(I_{z z}-I_{x x}\right)}{I_{y y}}\left[p+p_{T} \cos \psi \cos \theta+q_{T} \sin \psi \cos \theta-r_{T} \sin \theta\right] \\
& {\left[r+p_{T}(\cos \psi \sin \theta \cos \phi+\sin \psi \sin \phi)+q_{T}(\sin \psi \sin \theta \cos \phi-\cos \psi \sin \phi)+r_{T} \cos \theta \cos \phi\right]} \\
& +r\left[p_{T} \cos \psi \cos \theta+q_{T} \sin \psi \cos \theta-r_{T} \sin \theta\right]-\dot{q}_{T}(\sin \psi \sin \theta \sin \phi+\cos \psi \cos \phi) \\
& -p\left[p_{T}(\cos \psi \sin \theta \cos \phi+\sin \psi \sin \phi)+q_{T}(\sin \psi \sin \theta \cos \phi-\cos \psi \sin \phi)\right. \\
& \left.+r_{T} \cos \theta \cos \phi\right]+\dot{p}_{T}(\sin \psi \cos \phi-\cos \psi \sin \theta \sin \phi)-\dot{r}_{T} \cos \theta \sin \phi \\
& \dot{r}=\frac{\left(I_{x x} \mathcal{N}+I_{x z} \mathcal{L}\right)}{\left(I_{x x} I_{z z}-I_{x z}^{2}\right)} \\
& +\frac{\left(I_{y y} I_{x z}-I_{x x} I_{x z}-I_{z z} I_{x z}\right)}{\left(I_{x x} I_{z z}-I_{x z}^{2}\right)}\left[r+p_{T}(\cos \psi \sin \theta \cos \phi+\sin \psi \sin \phi)\right. \\
& \left.+q_{T}(\sin \psi \sin \theta \cos \phi-\cos \psi \sin \phi)+r_{T} \cos \theta \cos \phi\right] \\
& {\left[q+p_{T}(\cos \psi \sin \theta \sin \phi-\sin \psi \cos \phi)+q_{T}(\sin \psi \sin \theta \sin \phi+\cos \psi \cos \phi)\right.} \\
& \left.+r_{T} \cos \theta \sin \phi\right] \\
& +\frac{\left(I_{x x}^{2}-I_{x x} I_{y y}+I_{x z}^{2}\right)}{\left(I_{x x} I_{z z}-I_{x z}^{2}\right)}\left[p+p_{T} \cos \psi \cos \theta+q_{T} \sin \psi \cos \theta-r_{T} \sin \theta\right] \\
& {\left[q+p_{T}(\cos \psi \sin \theta \sin \phi-\sin \psi \cos \phi)\right.} \\
& \left.+q_{T}(\sin \psi \sin \theta \sin \phi+\cos \psi \sin \phi)+r_{T} \cos \theta \sin \phi\right] \\
& +p\left[p_{T}(\cos \psi \sin \theta \sin \phi-\sin \psi \cos \phi)\right. \\
& \left.+q_{T}(\sin \psi \sin \theta \sin \phi+\cos \psi \cos \phi)+r_{T} \cos \theta \sin \phi\right] \\
& -q\left[p_{T} \cos \psi \cos \theta+q_{T} \sin \psi \cos \theta-r_{T} \sin \theta\right]-\dot{p}_{T}(\cos \psi \sin \theta \cos \phi+\sin \phi \sin \phi) \\
& -\dot{q}_{T}(\sin \psi \sin \theta \cos \phi-\cos \psi \sin \phi)-\dot{r}_{T} \cos \theta \cos \phi
\end{aligned}
$$


The moment has two main components; due to aerodynamic forces and due to the thrust, thus

$$
\begin{aligned}
\mathcal{L} & =\frac{1}{2} \rho V_{R}^{2} \mathcal{S}_{R} b C_{\mathcal{L}}-\Delta_{z} T_{y}+\Delta_{y} T_{z} \\
\mathcal{M} & =\frac{1}{2} \rho V_{R}^{2} \mathcal{S}_{R} c C_{\mathcal{M}}-\Delta_{z} T_{x}-\Delta_{x} T_{z} \\
\mathcal{N} & =\frac{1}{2} \rho V_{R}^{2} \mathcal{S}_{R} b C_{\mathcal{N}}-\Delta_{y} T_{x}+\Delta_{x} T_{y}
\end{aligned}
$$

where $b$ is the wingspan, $c$ is the cord length of the receiver aircraft, and $\left(\Delta_{x}, \Delta_{y}\right.$, $\Delta_{z}$ ) are the moment arms of the thrust in the body frame of the receiver (see Fig. ??). The aerodynamic moment coefficients are

$$
\begin{aligned}
C_{\mathcal{L}} & =C_{\mathcal{L} 0}+C_{\mathcal{L} \delta_{a}} \delta_{a}+C_{\mathcal{L} \delta_{r}} \delta_{r}+C_{\mathcal{L} \beta} \beta+C_{\mathcal{L} p} \frac{b}{2 V_{R}} p_{r e l}+C_{\mathcal{L} r} \frac{b}{2 V_{R}} r_{r e l} \\
C_{\mathcal{M}} & =C_{\mathcal{M} 0}+C_{\mathcal{L} \alpha} \alpha+C_{\mathcal{L} \delta_{e}} \delta_{e}+C_{\mathcal{M} q} \frac{c}{2 V_{R}} q_{r e l} \\
C_{\mathcal{N}} & =C_{\mathcal{N} 0}+C_{\mathcal{N} \delta_{a}} \delta_{a}+C_{\mathcal{N} \delta_{r}} \delta_{r}+C_{\mathcal{N} \beta} \beta+C_{\mathcal{N} p} \frac{b}{2 V_{R}} p_{r e l}+C_{\mathcal{N} r} \frac{b}{2 V_{R}} r_{r e l}
\end{aligned}
$$

where $\left(p_{r e l}, q_{r e l}, r_{r e l}\right)$ are components of the angular velocity of the aircraft relative to the surrounding air. When the aircraft is in a vortex field as in the case of tanker's trailing wake vortex field, these angular velocity components will be different from the angular velocity relative to the tanker. 


\section{REFERENCES}

[1] Park, S., Avionics and Control System Development for Mid-Air Rendezvous of Two Unmanned Aerial Vehicles, Ph.D. thesis, Massachusetts Institute of Technology, February 2004.

[2] Yoshimasa Ochi, T. K., "Flight Control for Automatic Aerial Refueling via PNG and LOS Angle Control," AIAA Guidance,Naviation, and Control Conference and Exhibit, August 2005.

[3] Department of Defence, Washington, Joint Doctrine and Joint Tactics, Techniques, and Procedures for Air Mobility Operations, joint publication 3-17 ed., April 2006.

[4] USAF, AFPAM 10-1403.

[5] Brian S. Burns, M., Autonomous Unmanned Aerial Vehicle Rendezvous for Automated Aerial Refueling, Master's thesis, Aeronautic and Astronautics, WrightPatterson Air Force Base,OH, March 2007.

[6] Smith, A., "PNG with Adaptive Terminal Guidance for Aircraft Rendezvous," Air Force Institute of Technology Presentation, 2006.

[7] Jourdan, D., Trajectory Design and Vehicle Guidance for a Mid-Air Rendezvous between Two Autonomous Aircraft, Master's thesis, Massachusetts Institute of Technology, June 2003.

[8] McGee, T. G. and Hedrick, J. K., "Path Planning and Control for Multiple Point Surveillance by an Unmanned Aircraft in wind," Proceeding of the 2006 American Control Conference, Minniapolis, Minnesota,, June. 2006. 
[9] Derek R. Nelson, D. Blake Barber, T. W. M. and Beard, R. W., "Vector Field Path Following for Small Unmanned Air Vehicles," Proceeding of the 2006 American Control Conference, Minniapolis, Minnesota,, June. 2006.

[10] Timothy G. McGee, S. S. and Hedrick, J. K., "Optimal paht planning in a constant wind with a bounded turning rate," AIAA Guidance,Naviation, and Control Conference and Exhibit, 2005.

[11] Venkataramanan, S. and Dogan, A., "Dynamic Effects of Trailing Vortex with Turbulence \& Time-varying Inertia in Aerial Refueling," to be presented in the 2004 AIAA AFM Conference, Providence, RI, Aug. 2004.

[12] Venkataramanan, S. and Dogan, A., "A MultiUAV Simulation for Formation Reconfiguration," Proceedings of the AIAA Modeling and Simulation Technologies Conference and Exhibit, Providence, RI, Aug 2004, AIAA paper 2004-4800.

[13] Dogan, A. and Venkataramanan, S., "Nonlinear Control for Reconfiguration of Unmanned-Aerial-Vehicle Formation," AIAA Journal of Guidance, Control and Dynamics, Vol. 28:4, Jul-Aug 2005, pp. 667-678.

[14] Dogan, A., Venkataramanan, S., and Blake, W., "Modeling of Aerodynamic Coupling Between Aircraft in Close Proximity," AIAA Journal of Aircraft, Vol. 42:4, Jul-Aug 2005, pp. 941-955.

[15] Major Michael W. Leushen, M. P. W., "KC-10 Air Refueling Rendezvous without Electronic Emission," Tech. rep., Air Command and Staff College Air University, 1988.

[16] Venkataramanan, S., Dynamics and Control of Multiple UAVs Flying In Close Proximitry, Master's thesis, The University of Texas at Arlington, Arlington, TX, August 2004. 
[17] Kim, E., Control and Simulation of Relative Motion for Aerial Refueling in Racetrack Maneuver, Master's thesis, The University of Texas at Arlington, Arlington, TX, May 2007. 


\section{BIOGRAPHICAL STATEMENT}

Jane-wit Kampoon was born in Bangkog, Thailand, in 1972. He received his B.Eng degree in Aeronautical and Aviation Engineering ( $2^{\text {nd }}$ class honor) from Royal Thai Air Force Academy(RTAFA), Thailand, in 1996. In 2000, he won a Royal Thai Government(RTG) scholarship pursuing master degree and received his M.Eng degree in Mechatronics from Asian Institute of Technology, Thailand. From 1996 to 2007, he worked for Royal Thai Air Force (RTAF), Aeronautical Engineering Department, Royal Thai Air Force Academy as a full time Instructor. In Fall 2007, he won a scholarship from RTAF pursuing his second master Degree at The University of Texas at Arlington in Aerospace Engineering. 